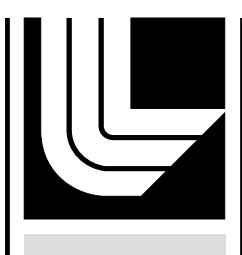

LAW RENCE LIVERMORE N A T IO N A L LABORATORY

Progress on the Flash X-Ray Optical Transition Radiation Diagnostic

V. Tang, T. Houck, C. Brown

March 31, 2008 
This document was prepared as an account of work sponsored by an agency of the United States government. Neither the United States government nor Lawrence Livermore National Security, LLC, nor any of their employees makes any warranty, expressed or implied, or assumes any legal liability or responsibility for the accuracy, completeness, or usefulness of any information, apparatus, product, or process disclosed, or represents that its use would not infringe privately owned rights. Reference herein to any specific commercial product, process, or service by trade name, trademark, manufacturer, or otherwise does not necessarily constitute or imply its endorsement, recommendation, or favoring by the United States government or Lawrence Livermore National Security, LLC. The views and opinions of authors expressed herein do not necessarily state or reflect those of the United States government or Lawrence Livermore National Security, LLC, and shall not be used for advertising or product endorsement purposes.

This work performed under the auspices of the U.S. Department of Energy by Lawrence Livermore National Laboratory under Contract DE-AC52-07NA27344. 
LLNL-TR-402621

\title{
Progress on the Flash X-Ray Optical Transition Radiation Diagnostic
}

V. Tang, T. Houck, and C. Brown

National Security Engineering Division, LLNL

\begin{abstract}
This document summarizes the Flash X-Ray accelerator (FXR) optical transition radiation (OTR) spot-size diagnostics efforts in FY07. During this year, new analysis, simulation, and experimental approaches were utilized to interpret OTR spot data from both dielectric foils such as Kapton (VN type) and metal coated foils. Significant new findings of the intricacies involved in the diagnostic and of FXR operational issues were achieved. Geometry and temperature based effects were found to affect the beam image profiles from the OTR foils. These effects must be taken into account in order to deduce accurately the beam current density profile.
\end{abstract}

Portions of this work are expected to be published in Nuclear Instruments and Methods, Section A. 


\section{Introduction}

This document summarizes the Flash X-Ray (FXR) accelerator [1] optical transition radiation (OTR) spot-size diagnostics efforts in FY07. During this year, new analysis, simulation, and experimental approaches were utilized to interpret OTR spot data from both dielectric foils such as Kapton (VN type) and metal coated foils. Significant new findings of the intricacies involved in the diagnostic and of FXR operational issues were achieved.

The objective of the diagnostic is to provide accurate beam profile and emittance data for FXR in order to diagnose different accelerator magnetic transports and energy profiles. Optimum tunes ultimately reduce the beam spot size at the bremmstralung target and maximize radiograph resolution. In this introductory Section, the FXR beam imaging diagnostics and previous work on interpreting data from them are quickly reviewed, followed by executive summaries of the new FY07 results which include detailed outlines of the entire report.

Readers unfamiliar with OTR and Cerenkov imaging should review references [2-5]; a brief synopsis is given here. In general, OTR occurs when charged particles like electrons move across a boundary, such as a foil. The emission is not isotropic and increases in anisotropy with particle energy. Figure 1 illustrates the characteristics of OTR and a simple foil diagnostic. A typical setup consists of a foil with its normal vector angled 45 degrees with respect to the electron beam and a camera setup to view the intense emission emitted preferentially in the direction of specular reflection (if the electron beam was replaced with a beam of light), denoted here as the specular vector. Depending on the diagnostic implementation, OTR can be used to image the current profile, energy, and divergence of a relativistic electron beam. As the electron beam penetrates the foil, intense Cerenkov radiation is generated which can also be used for diagnostic purposes. Cerenkov radiation is characterized by a forward emission cone inline with the electron beam, is independent of the angle between the foil and the beam, and can be significantly more intense than OTR. This report primarily concerns OTR 
emission. Typically metallic foils are used for OTR which easily prevents internally emitted and scattered optical Cerenkov radiation from reaching an OTR diagnostic; however, depending on the diagnostic setup and the Cerenkov emission angle, a metal foil is not necessarily required since the Cerenkov light can miss the OTR diagnostic by geometric reasons alone.

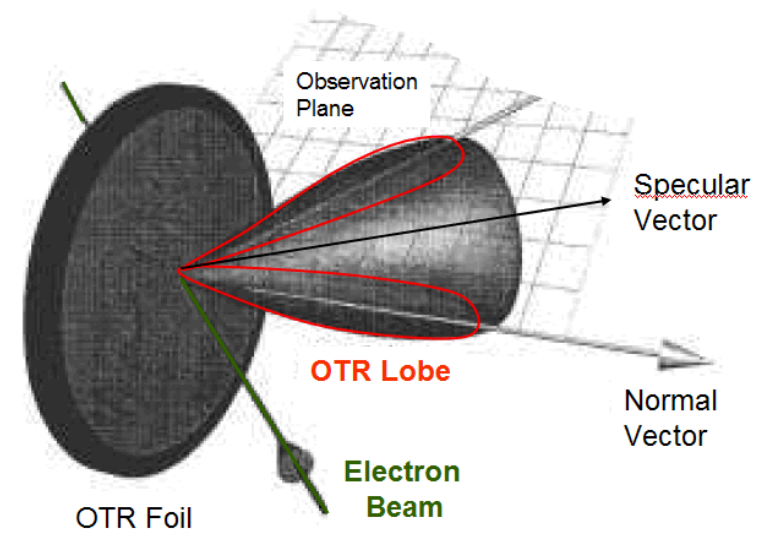

Figure 1: Schematic of OTR emission. For relativistic electron beams, the emission is anisotropic and concentrated roughly in the cone defined by the lobes. For FXR electron beams at $17.5 \mathrm{MeV}$, the cone or lobes are angled $\sim 1.7$ degrees from the specular vector of the electron beam. The angular "thickness" of the cone walls or lobes is typically $\sim 4$ degrees. Modified from [6].

On FXR, the imaging diagnostics [7-9] consist primarily of two different setups. The first setup is colloquially referred to as the Wiggle Probe [7] and allows imaging of the electron beam in between acceleration gaps. The Wiggle Probe is illustrated in Figure 2 and currently uses a slightly roughened Kapton (VN type, rough side facing the mirror or away from the beam) angled 75 degrees away from the beam based on angle $\phi$ in the Figure. Unspecified beam-foil angles in this report will always referred to $\phi$. Unlike traditional OTR diagnostics, the Wiggle Probe is setup to image the back of the foil relative to the beam, which likely results in it seeing a combination of mostly scattered Cerenkov and some optical transition radiation. The current Wiggle Probe design does not allow for operation at the typical $\phi=45$ degrees used for OTR imaging, nor does it use a conventional metallic foil for fears of increasing the possibility of breakdowns in the accelerator gaps. The second imaging setup, where the discussed experiments in this report were performed, is stationed at the "Diagnostic Cross", a chamber with six ports 
located approximately 2 meters after the accelerator exit. Here, significant flexibility is available and the beam can be imaged using Al-coated foils (typically $\sim 300 \AA$ coating on $0.04 \mathrm{~cm}$ quartz), roughened Kapton foils (VN type, typically $0.013 \mathrm{~cm}$ thick) or frosted quartz foils (typically $0.04 \mathrm{~cm}$ thick) at user-set angles. This second setup is illustrated in Figure 3. The flexibility of the diagnostic cross setup allows users to perform OTR specific experiments, such as experimentally determining light intensities from foils angled at 45 versus 75 degrees. The two diagnostics can operate in either angular or spatial imaging modes; this report concerns spatial or spot imaging and primarily data from the Diagnostic Cross with foils angled at 45 degrees. Lastly, the dielectric foils used here are typically slightly roughened or frosted in order to isotropize and sometimes increase the signal to the camera; however, this also increases the likelihood that backscattered Cerenkov radiation will be imaged and overall can make detailed modeling of the emission difficult. The former point is further addressed and discussed at the end of this Section. In short for purposes here it is taken that OTR is the dominant component even for the roughened dielectric foils considered here. For smooth and lightly roughened dielectric foils, as shown later, this is the case since the Cerenkov radiation in the type of experiments discussed later should not have entered the diagnostic viewing cone, assuming geometric optics. Overall, the key results of this report do not change even if Cerenkov radiation is significant for some of the discussed experiments. 
LLNL-TR-402621

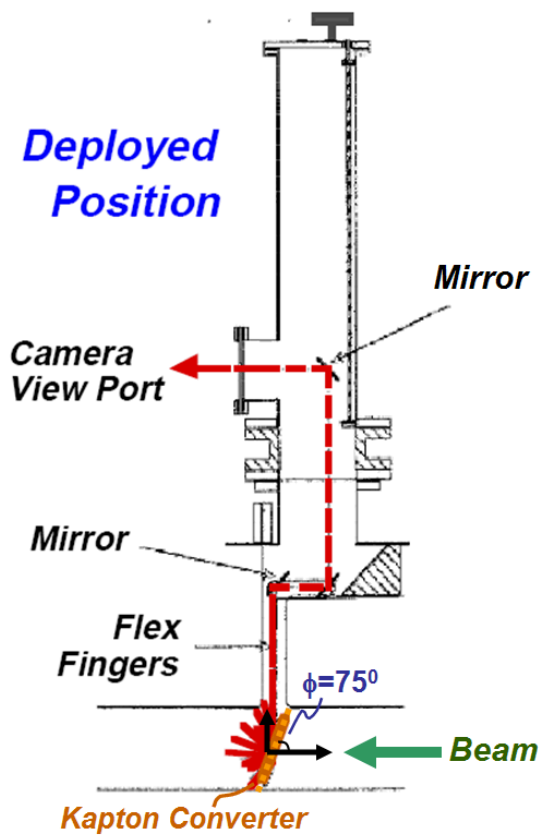

Figure 2: Schematic of FXR OTR Wiggle Probe Diagnostic. Modified from [7].

The OTR optical system consists of a $60 \mathrm{~mm} \mathrm{f} / 2.8 \mathrm{D}$ Nikon collection lens $\sim 25 \mathrm{~cm}$ away from a rotatable $\sim 11 \mathrm{~cm}$ diameter OTR foil. The signal is coupled via optical fibers to a fast CCD camera typically operated with gate widths of $\sim 2 \mathrm{~ns}$. The images shown in this report were taken with a Princeton Instruments PI-MAX Gen II intensified CCD camera system. More information on the camera system can be found in reference [10]. Discussed shot numbers are FXR facility references for beam pulses into the accelerator. All data acquired for a specific pulse is reference by a shot number. The beam is guided through the injector and accelerator by a series of magnetic solenoids and dipoles. Reference [11] lists the magnetic locations and operating currents for specific tunes mentioned in this report, e.g. REX06. 
LLNL-TR-402621

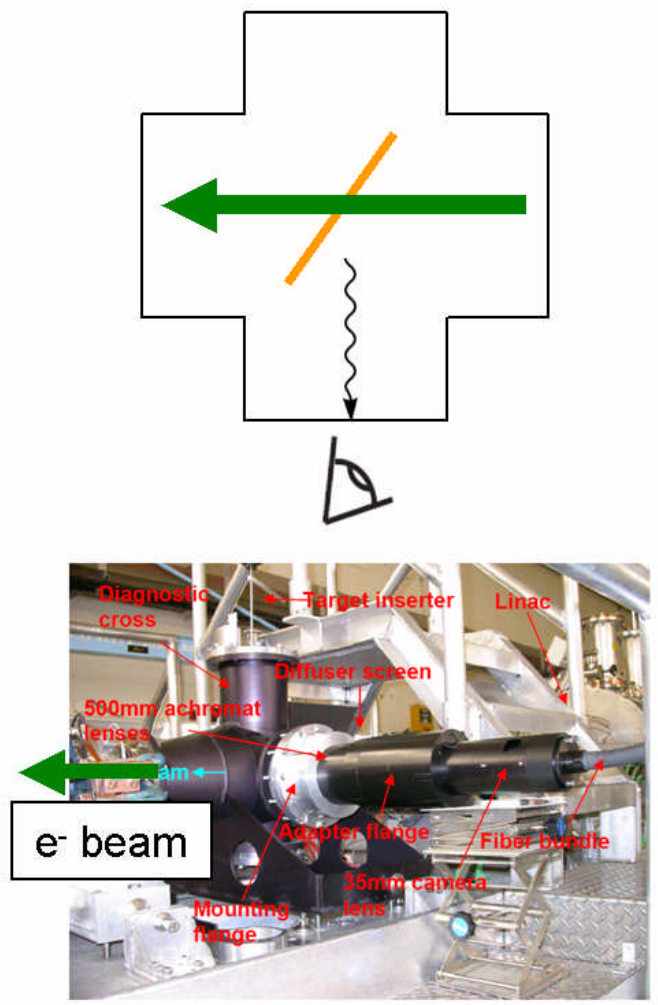

Figure 3: Schematic and photo of the OTR diagnostic setup at the Diagnostic Cross. The green arrow indicates the FXR electron beam. Modified from [9].

Previously, the intensity profile from spot or spatial image OTR data from roughened or frosted foils were assumed to correspond directly to the beam spatial current density profile through a simple multiplicative constant. In other words it was assumed that the OTR spot profile was also relatively the beam current density profile and no diagnostic response function was required for a relative measurement of the beam current density profile or beam radius. However, T. Houck and A. Paul [12] found that this interpretation of the OTR spot profile data from Kapton foils on both the Wiggle Probe and at the diagnostic cross did not give beam profiles that matched results from their beam transport simulations. They therefore suggested that temperature based permittivity changes from beam heating affected the OTR emission and resulted in a diagnostic response function that varied with the foil temperature profile and hence spatially across the OTR spot image. Thus, the OTR spot profile was not simply a constant multiplied by the beam current density profile. Furthermore, Houck and others [7] found that for the Kapton shots taken at both the Wiggle Probe and Diagnostic Cross, the total counts, i.e. 
the sum of the image pixel values after background subtraction, from spot imaging rose as the spot image RMS radius increased. This observation supports Houck's varying permittivity argument since the total counts per incident electron should be conserved assuming a roughened foil that effectively gives isotropic or randomized OTR emission. Lastly, assuming an OTR emission that varied with the permittivity $\varepsilon$ via $|1-\varepsilon|^{2}$, Houck completed a preliminary isotropic spot-size OTR model with DC Kapton and quartz permittivity data which qualitatively simulated a total counts curve that increased with spot RMS radius for Kapton foils. Figure 4 shows a simulation of the total counts as a function of beam RMS radius completed at the beginning of FY07 for different types of beam profiles using Houck's model and assuming a room temperature foil before the pulse. The rise in counts with beam RMS radius is primarily due to a decreased permittivity in the center of the foil from beam heating which results in lowered OTR emission from the center of the foil per incident electron. Based on the DC permittivity data, frosted quartz foils should not be affected. Overall, Houck concluded that a detailed diagnostic simulation was therefore necessary to accurately interpret the FXR spot-size OTR data from dielectric, specifically Kapton, foils and retrieve the real beam radius or profile. It is important to reiterate that Houck's preliminary model uses the beam RMS radius while the observed trend is with spot RMS radius and that it assumes completely randomized OTR emission. The new synthetic diagnostic or diagnostic simulation would be used to predict the spot RMS radius or profile given user inputs, could be used to predict the total counts as a function of spot RMS radius, and ultimately provide the correlation between beam RMS radius and spot RMS radius.

A directly related concern for interpreting the spot-size data is the method by which the Kapton and quartz foils, with poor thermal conductivities, cooled themselves in-between shots, and if precise interpretation of these OTR data require very accurate temperature models of the foils with shot-to-shot thermal accounting. In theory, metallic foil OTR do not suffer from these temperature related effects and are much easier to model, but because of the need to use a flexible dielectric foil for the Wiggle Probe diagnostic and the desire to minimize conductive material in the overall beamline, materials such as Kapton were pursued. 


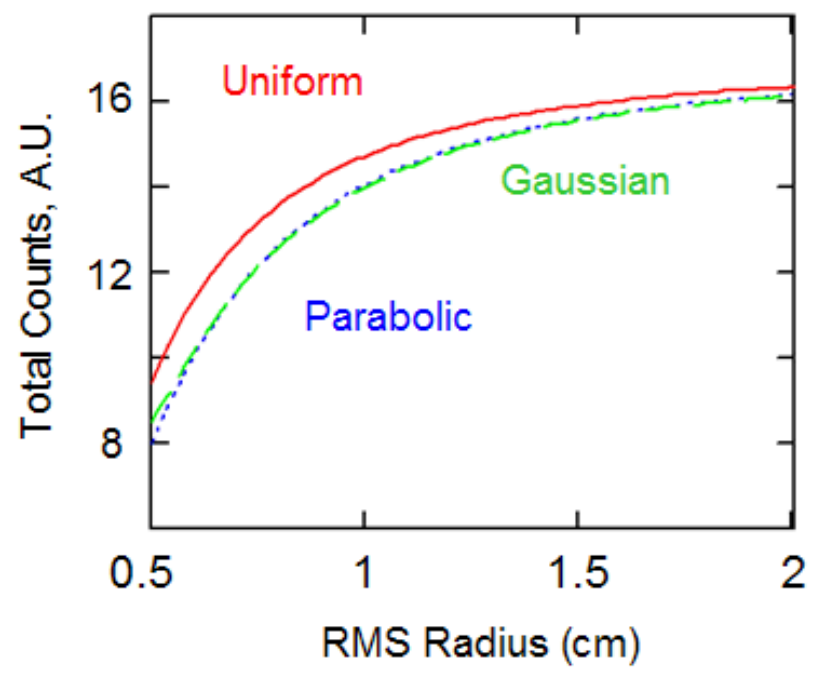

Figure 4: Simulation of total spot counts as a function of RMS beam radius for different types of beam profiles carrying the same total electron current using Houck's model.

For this year, noticeably better understanding of these OTR spot-size interpretation problems was achieved and some answers to the above question were found. More importantly, some repeatability issues on FXR were uncovered. A summary and introduction of the results are given below; the beam imaging research discussed here using dielectric foils at the Diagnostic Cross is applicable also to the Wiggle Probe diagnostic and recommendations for that diagnostic based on the research is included.

First, a new post-shot analysis package was written to facilitate analysis of the spot-size OTR SPE files [13]. This Matlab [14] toolset is an enhanced version of the analysis programs written by C. Brown and was used initially to verify the Kapton OTR trends that Houck and Paul uncovered previously. In the process, similar trends for the frosted quartz foils were also observed in the old dataset. This task moreover served to benchmark the new toolset package. A description of the code and benchmarking results is given in Section $\mathrm{I}$.

The question of foil cooling in-between shots was resolved by a new temperature model which took into account thermal radiation; because of the large surface area of the foils and their relative thinness, significant cooling occurs via thermal radiation even at temperatures less than $1000 \mathrm{C}$. The model suggests that for a series of repeat shots, the 


\section{LLNL-TR-402621}

foil temperatures reach a steady-state relatively quickly. In addition to OTR calculations, the model is already being used to prevent foil damage due to beam heating. Also, a series of Kapton heating experiments in a He furnace was performed to determine visually the effects of temperature induced changes. The model and results are described in Section II.

Experimentally, multiple series of repeat shots on FXR were done with both Kapton and frosted quartz in order to confirm at least qualitatively temperature based effects on the OTR spot data. For a benchmark, the same series of repeat shots with an Al metal foil, which should not be affected by temperature based permittivity effects, was performed. Assuming that significant temperature related OTR effects exist, these three series of repeat shots should produce moderately different RMS radii for the same FXR settings, and in some cases noticeable variation shot-to-shot for the dielectric foils. Ideally, accurate modeling and understanding of OTR from dielectric foils would be confirmed if the results, specifically the RMS radius and total counts from the three run series, could be reconciled shot-to-shot in each series, and respectively with the Al benchmark. The benchmarking of such a comprehensive simulation or synthetic diagnostic would then provide a correction between spot RMS radius and real beam RMS radius. The modeling effort accomplished so far is described in Section IV, summarized next. The data from the repeat shot series are still under analysis but some preliminary conclusions, including shot reproducibility issues on FXR, are available. The quartz data contains shot-to-shot OTR spot variations which could be explained by temperature related OTR effects and a quartz permittivity at optical frequencies which declines noticeably with temperature; shot reproducibility however was identified as an issue for these data, especially for the first shots of the day. The Kapton experiments proved problematic due to signal levels and foil damage, but nevertheless qualitatively matched expectations using the radiation model in Section II. Based on the assumptions above and the available data from the quartz repeat runs and the Al benchmarking runs, noticeable evidence suggests that there are shot reproducibility issues on FXR in the case when warm-up shots are not performed. These repeatability concerns could be partly the reason why previous attempts to compare beam radii with simulations were inconclusive. Lastly, some more 


\section{LLNL-TR-402621}

potential issues for dielectric foils were identified this year during analysis of these experimental data. For example, a related and potential problem unexamined so far for the FXR OTR diagnostic is that the foil itself, independent of material, might be significantly perturbative due to back-streaming ions [15] or other mechanisms. Additional effects such as dielectric foil charging have also not been studied. These phenomena, if they are significant, could modify some of the conclusions above. The experimental results and observations are discussed fully in Section III.

In regards to simulations, a synthetic OTR diagnostic simulation for smooth dielectric and metallic foil was created which identified and resolved some OTR spot interpretation issues. The ultimate goal of this tool is to provide the interpretative modeling necessary to determine accurate beam profile data from OTR spot data, with verification of the simulations involving dielectric foils coming from the benchmarking experiments such as the ones discussed in Section III. The tool is preliminarily operational and some results are available. Concerning those results, a geometric factor was found in the diagnostic response function which varies with radius and plays a role in interpreting both smooth dielectric and metallic foil OTR spot images. This is in addition to temperature based effects. The geometric effect affects the radius interpretation of the spot data more than the temperature based effects. However, the temperature effects are clearly seen in a simulation of the total counts. Spot RMS radius versus real beam RMS radius plots for cold beams at a waist are now available for both metallic and Kapton foils. So far, fortuitously, the spot RMS radii and the beam RMS radii in the simulations are not too far apart $(\sim 10 \%)$ when an optimum optical setup with low f-number is used. In other cases, such as an optical setup with high f-number, the synthetic diagnostic results show that the observed spot-size and radii can be noticeably larger than the real beam radii, due to both the anisotropic nature of OTR and the studied temperature effects. Lastly, a qualitative match was found between an experimental and simulated total counts versus spot RMS radius curve for some of the Kapton data. Although a prediction of the total counts is not strictly required as long as an accurate spot radius can be predicted given a beam radius, a good comparison of experimental and simulated total counts versus spot RMS radius would serve as an important benchmarking exercise and also explain some 
of the observed experimental total counts trends. Lastly, for the cases examined, the emission intensity versus permittivity scaled with a more complicated quadratic than the previously assumed $|\varepsilon-1|^{2}$. A set of challenges identified from this beginning work on the dielectric modeling portions of the code is again the need for a foil which is either completely roughened or smooth for accurate simulations, and the lack of accurate permittivity data as a function of temperature for the dielectric foils of interest. Section IV documents this new synthetic diagnostic and the work done so far.

The last portion, Section V gives a conclusion, list of suggested work for FY08, and summarizes recommendations for future diagnostic experiments. A key result is that based on the level of effort required to operate and accurately interpret a one-foil dielectric OTR diagnostic, it is recommended that future OTR spot experiments and measurements be conducted only with metallic foils or coatings, such as Al. Examination of the feasibility of operating the Wiggle Probe at 45 degrees with Al coated Kapton is also recommended since the Wiggle Probe is most prone to interpretation issues. Further understanding of dielectric foil imaging, especially at the Wiggle Probe, could be achieved by adding Cerenkov radiation to the simulation if metallic foils are simply not feasible. More importantly, repeatability issues on FXR should be addressed and diagnosed with metallic foils before dielectric foils, especially Kapton, are attempted again.

Finally, for the purposes of this report, it has been assumed that the dominant radiation imaged is OTR, and not Cerenkov radiation for the roughened or frosted dielectric foils. This assumption is supported by a simple geometric optical analysis of the typical experiment setup assuming a smooth foil. For a foil angled at 45 degrees and a camera setup with a relatively small viewing cone to view the OTR along the specular vector, Cerenkov radiation would only be visible if the internally backscattered light refracts out of the front layer of the foil aligned approximately with the specular vector. Since the viewing cone angular width and sightline tilts off of the specular vector for the FXR OTR diagnostic are small, typically less than 5 degrees for both cases, this simple approximation for the camera optics suffices for this analysis. Figure 5 illustrates the 


\section{LLNL-TR-402621}

ray-tracing involved for a Kapton foil at optical frequencies, using an index of refraction n of $\sim 1.8$ and a permittivity $\varepsilon$ of $\sim 3.5$, and shows that backscattered Cerenkov radiation should not be detected. In fact, the backscattered light that makes it back through the front of the foil is refracted away from the specular vector. The emission angle for Cerenkov radiation is given by $\cos ^{-1}\left(1 / \beta \varepsilon^{1 / 2}\right)$ and ranges from $\sim 45-60$ degrees for the quartz and Kapton foils of concern due to changes in permittivity as a function of material and frequency. Varying the material parameters used for the analysis in Figure 5 still does not result in detectable refracted Cerenkov radiation. Now, roughened and frosted foils used previously at the Diagnostic Cross were used again for the FY07 experiments; the experiments done as discussed in Section III do not explicitly depend on having smooth dielectric foils and hence smooth foils were not used in order to maintain the same experimental setup as before. In theory if the rough or frosted surface completely randomizes the OTR and Cerenkov radiation, the diagnostic would likely be dominated by the latter. However, a simple scattering experiment with the roughened Kapton and frosted quartz foil using a laser gave an effective scattering angle of only 510 degrees. Given that $\sim 30$ degrees or more of additional scattering is required for the Cerenkov to reach the imaging setup in Figure 5, OTR could still be dominant over Cerenkov radiation for the experiments in this report even accounting for finite angular widths of the viewing and emission cones, and the significant amount of Cerenkov radiation produced. Also, because the scattering from the rough foil surface is small, the preliminary detailed simulation results for smooth dielectric foils assuming OTR emission in Section IV are still qualitatively relevant.

It is not feasible to accurately simulate the emission response from a dielectric foil if OTR and Cerenkov radiation are both important due to foil roughness and if the permittivity type effects are significant, since both the OTR and Cerenkov radiation have different angular and permittivity dependencies, and an accurate model of the foil roughness would be required. Qualitatively, if it turns out that Cerenkov radiation is dominant and that the radiation is truly isotropic due to foil roughness, the total counts as a function of spot RMS radius should decrease with increasing radius for a foil permittivity that decreases with temperature, since Cerenkov emission is inversely 
proportional to permittivity. This has not been seen. The analysis programs and methods described in this report could be easily adapted for Cerenkov radiation if required, and in fact, for shot-to-shot temperature based permittivity effects, discussed in Section III, the predicted experimental trends for repeat shots neatly do not change between OTR or randomized Cerenkov radiation.

Of course, for $\mathrm{Al}$ coated foils of several hundred Angstroms coating thickness, the visible radiation imaged from the front of the foil is clearly just OTR since the Cerenkov from within the foil can not penetrate the Al coating. Al-coated or Al foils for spot imaging is clearly the preferred choice. Anyhow, it should be noted that the above potential Cerenkov and OTR issue does not change the main conclusions of this report summarized above and in the conclusion.

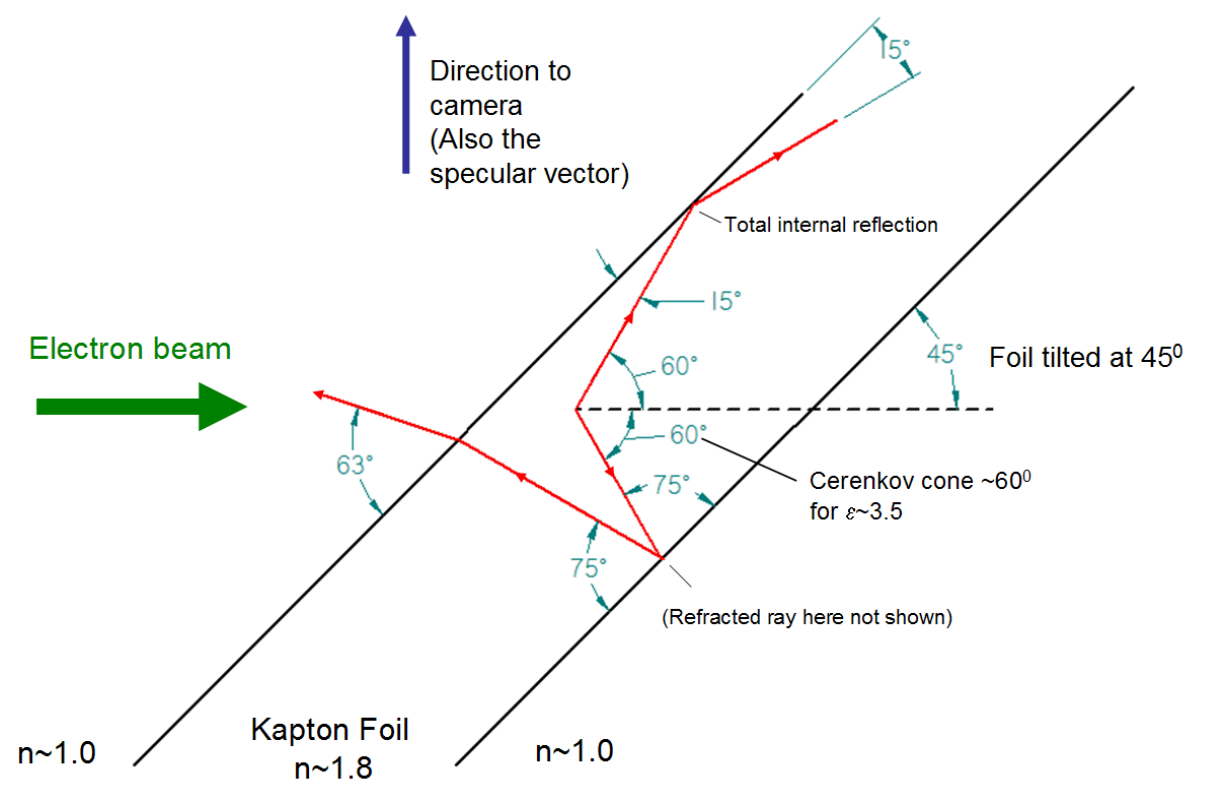

Figure 5: Analysis of backscattered and refracted Cerenkov radiation for a smooth Kapton foil. Assuming a camera sightline aligned with the specular vector the raytracing shows that backscattered Cerenkov should not be detected. The foil thickness is clearly exaggerated for clarity. 
LLNL-TR-402621

Introduction References

[1] B. Kulke et al., "Design of a $20 \mathrm{MeV}, 4 \mathrm{kA}$ Linear Induction Accelerator", IEEE Conf. Plasma Science, 1980.

[2] L. Frank and V. Ginzburg, J. Phys. USSR 9, 353, 1945.

[3] M. L. Ter-Mikaelian, High Energy Electromagnetic Processes in Condensed Media, Wiley-Interscience, New York, 1972.

[4] V. E. Pafomov, Proceedings P.N. Lebedev Physics Institute, 44, pgs. 25-157, 1971.

[5] L. Wartski et al., J. Appl. Phys. 44, 8, 1975.

[6] C. Vermare and D.C. Moir, LA-UR-01-1171, LANL Technical Report, 2001.

[7] M. Ong et al., "FXR fast beam imaging diagnostics", $12^{\text {th }}$ IEEE Pulse Power Conf., 1999. Also M. Ong et al., UCRL-JC-134615, LLNL Technical Report, 1999.

[8] G. P. Le Sage, UCRL-ID-153254, LLNL Technical Report, 2002.

[9] J.S. Jacob et al., UCRL-TR-214037, LLNL Technical Report, 2005.

[10] Princeton Instruments, http://www.piacton.com/

[11] A.C. Paul, UCRL-TR-212069, LLNL Technical Report, 2005. Tables 34, 51, and 52.

[12] A.C. Paul, UCRL-TM-219436, LLNL Technical Report, 2006. Page 29.

[13]SPE is the image file format used by the control software from Princeton Instruments CCD camera.

[14] The MathWorks, http://www.mathworks.com/

[15] For example, Y-J. Chen et al, "Controlling Backstreaming Ions from X-ray Converter Targets with Time Varying Final Focusing Solenoidal Lens and Beam Energy Variation", $19^{\text {th }}$ Int. Linear Acc. Conf., 1998. 
LLNL-TR-402621

\section{Section I: Matlab post-shot toolset and new observations on old datasets}

The Kapton total counts versus radius trends observed by Houck and Paul were confirmed by a new post-shot analysis code. In addition, similar trends for even the quartz foils are observed.

The new toolset is an enhanced version of the programs written by Brown. Specifically, the toolset takes in a series of shots in an Excel namelist and outputs parameters such as image RMS radius, centroid location, and various plots such as image RMS radius versus total counts or total pixel counts after background subtraction. For each shot, the code first performs background subtraction; typically the automated option is utilized which results in a background value based on the average data from the rightmost column or vertical strip. A median filter is then applied and the centroid location of the beam image is determined. Next, a Gaussian fit is performed on the column of pixel data from the centroid of the image which determines the effective image RMS radius. Pixels on the fiducial holes from this column of data can be excluded from the fit. A theoretical or analytical total counts based on that Gaussian fit is calculated; this number is useful for comparison with the real total counts, especially for shots with the foil at 45 degrees, since it is more resilient to anomalies such as fiducial holes, image fall-off from the foil, and other types of noise that might not be taken into account by the background subtraction method above. For each shot, the parameters and fits are summarized in an output figure similar to Figure 1.1 with the data saved in a Matlab data file. A series of plots summarizing the major output parameters, such as radius versus shot number, are also made and automatically saved.

Using the toolset, previous runs with Kapton and quartz dielectric foils were analyzed and compared with Houck's SUICIDE [1] analyses. The comparisons are summarized in Figures 1.2-1.5 and support Houck's previous observations for Kapton. The quartz data also followed similar trends; this does not necessary contradict Houck's preliminary OTR model for quartz since it used DC permittivity data. In terms of the toolset, the 
benchmarking results are encouraging since different fitting and background subtraction methods were used by Houck compared with the Matlab toolset.
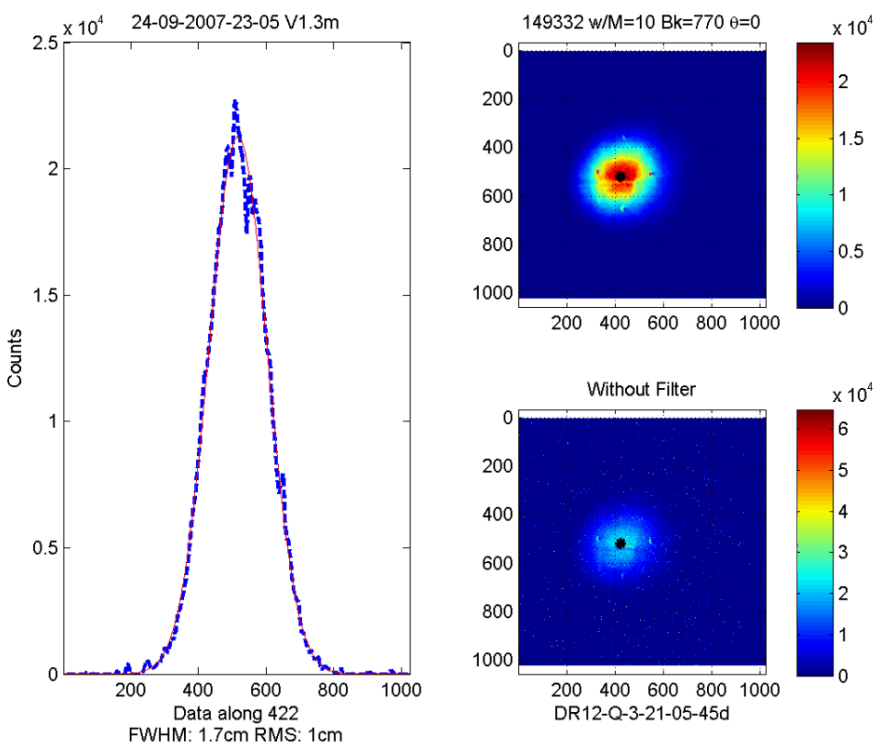

Figure 1.1: Spot-size analysis results from the Matlab post-shot toolset. The left side gives the vertical intensity readout based on the filtered image on the top right. The centroid given by the code is marked with a black dot.

Comparison of K45 Radii V1.3m

25-Sep-2007 20:48:42
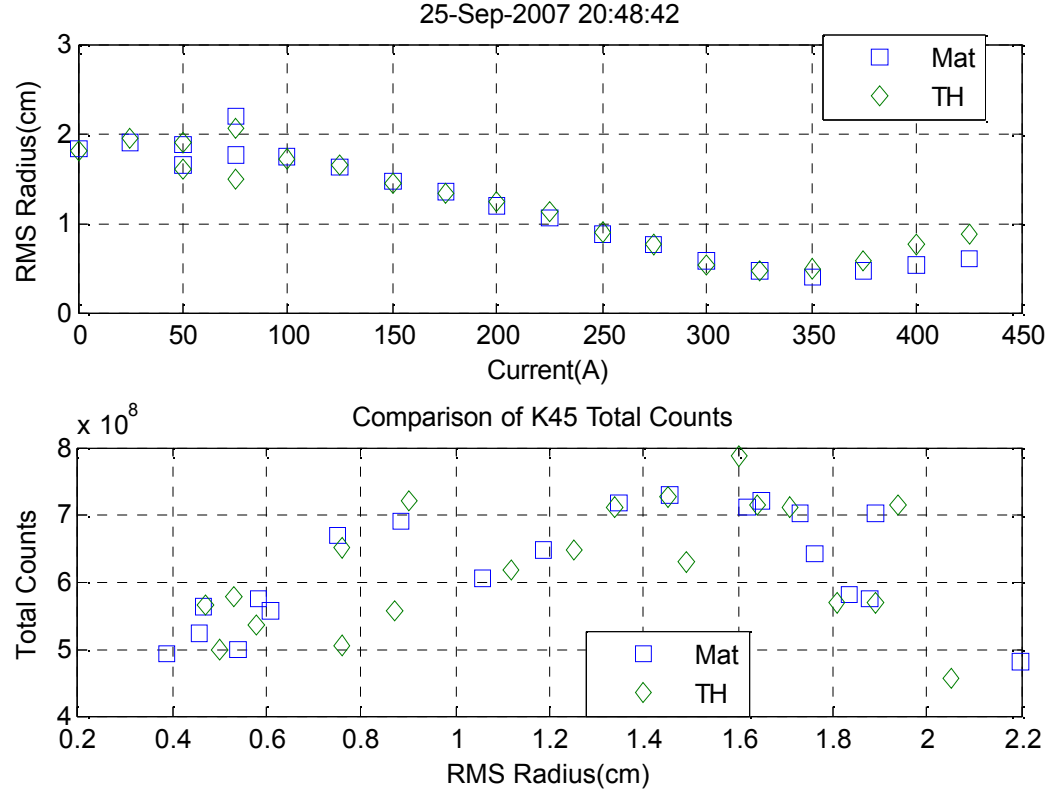

Figure 1.2: Comparison of the fitted spot-size radius and total counts data for shots 147839 to 147857 on Jan $21^{\text {st }}$, 2005. This series scanned DR1-DR2 magnet currents using a roughened 45 degree Kapton foil at the diagnostic cross. The legend "Mat" indicates results from the Matlab toolset with "TH" indicating Houck's Suicide analysis results. Although different fitting and background subtraction routines were utilized, the results are similar. 
LLNL-TR-402621
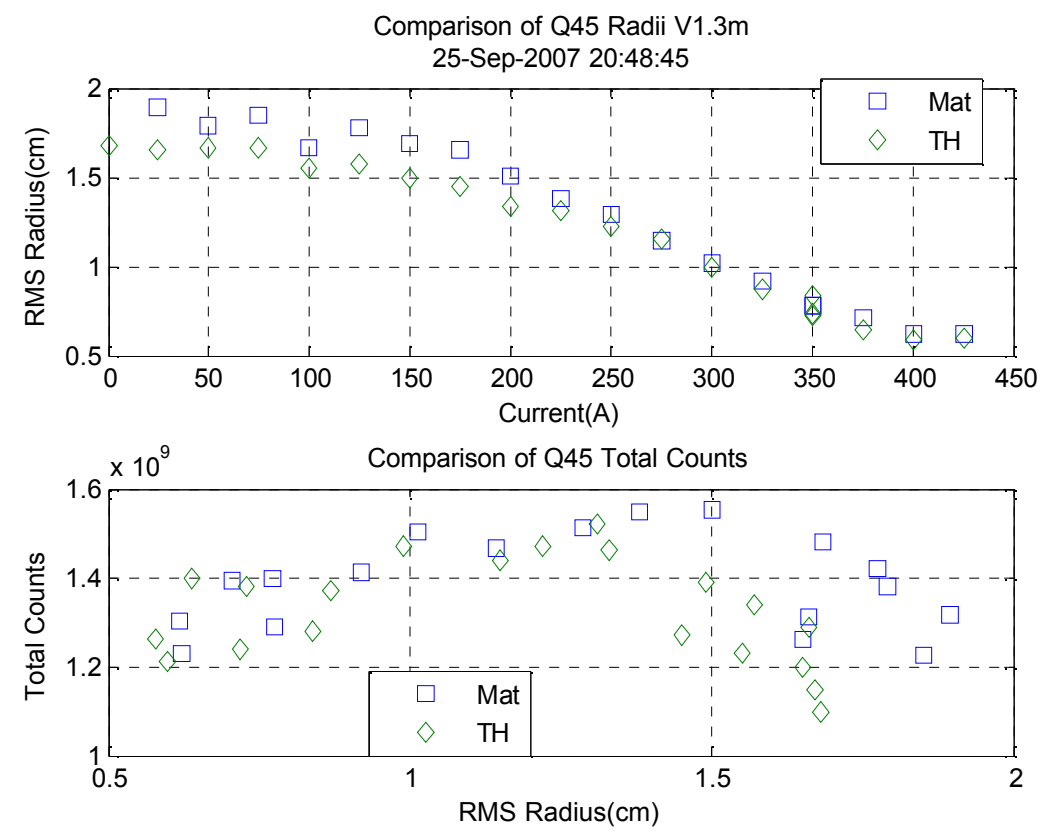

Figure 1.3: Comparison of the fitted spot-size radius and total counts data for shots 149321 to 149338 on Mar 21 ${ }^{\text {st }}, 2005$. This series scanned DR1-DR2 magnet currents using a frosted 45 degree Quartz foil at the diagnostic cross. The legend "Mat" indicates results from the Matlab toolset with "TH" indicating Houck's Suicide analysis results. Different fitting and background subtraction routines were utilized, giving slightly different radius answers at the lower currents. 
LLNL-TR-402621
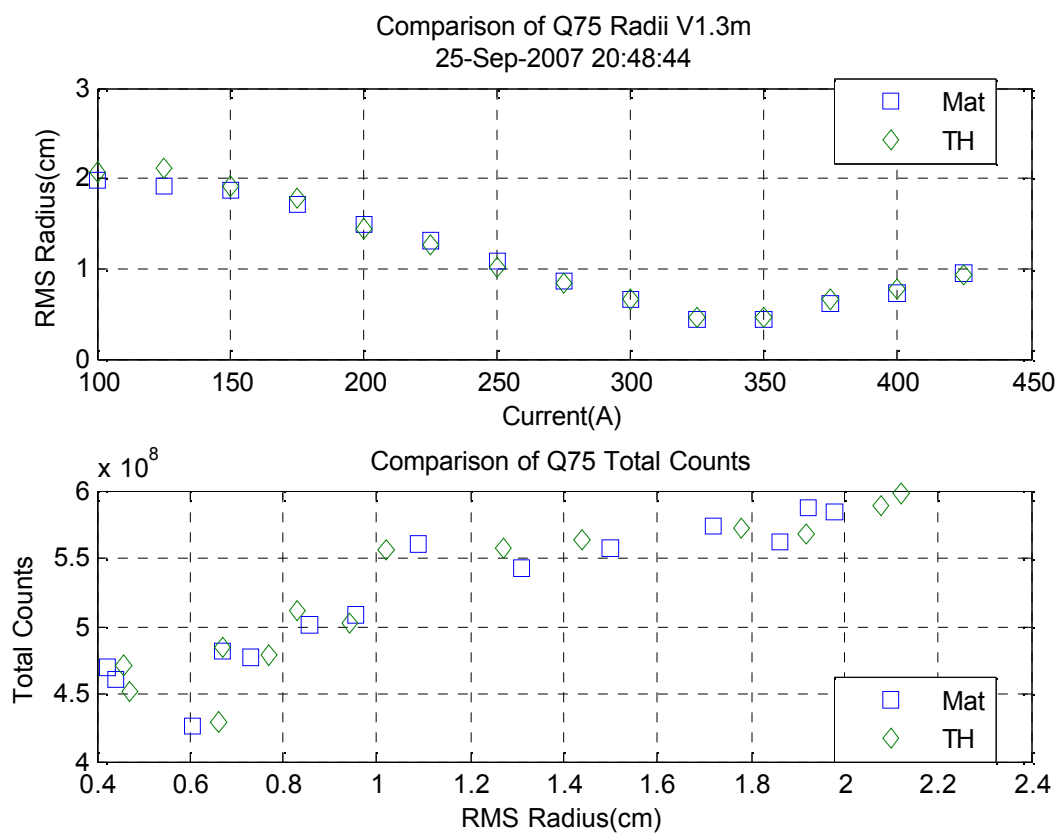

Figure 1.4: Comparison of the fitted spot-size radius and total counts data for shots 147135 to 147148 on Dec $17^{\text {th }}, 2004$. This series scanned DR1-DR2 magnet currents using a frosted 75 degree quartz foil at the diagnostic cross. The legend "Mat" indicates results from the Matlab toolset with "TH" indicating Houck's Suicide analysis results. Although different fitting and background subtraction routines were utilized, the results are remarkably close to each other. 


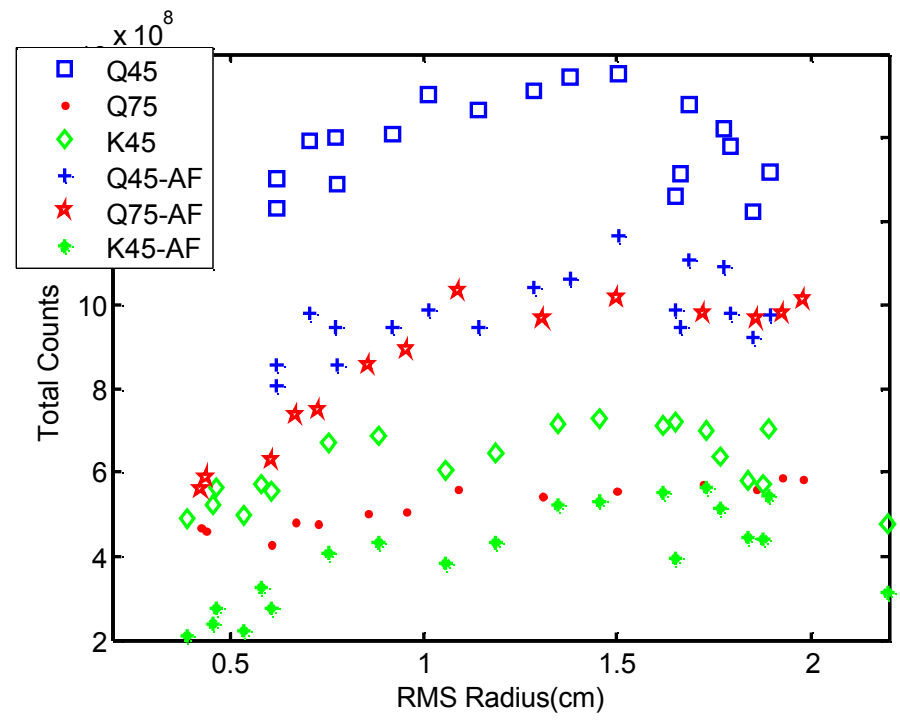

Figure 1.5: Summary of the total counts vs. spot RMS radius from the shots in Figure 1.2-1.4. 'AF' denotes total counts calculated form the analytical Gaussian fits performed in the toolset. For the Q45 cases, the drop in total counts at the larger radii is likely due to the beam falling off of the foil. The total counts from the analytical fits corrects for this type of issue which can obscure real trends in the data. Overall, this set of data indicates an increasing total counts as a function of fitted RMS radius for every shot series.

\section{Section I References}

[1] F. Chambers and B. Raymond, "Simple User Interface Connecting to an Integrated Data Environment (SUICIDE)”, LLNL. 


\section{Section II: Temperature Model for OTR Foils}

Previously, the temperature history of the FXR OTR foil and the cooling mechanism involved was not completely clear. Modeling and experiment efforts this year identified radiation cooling as the primary mechanism and provided useful estimates of the foil temperature during a shot series.

Section IIa: Beam heating and foil cooling in-between shots

During the shot, the beam instantaneously heats the OTR foil and results in a temperature profile given by:

$T(\vec{r}, t)=\int_{0}^{t} \frac{n_{e}(\vec{r}, t) v_{e} E}{c_{p}} d t+T(\vec{r}, 0)$

where $n_{e}$ is the electron density in $\# / \mathrm{cm}^{3}, v_{e}$ is the speed of the electrons or $\sim 3 \times 10^{10}$ $\mathrm{cm} / \mathrm{s} . \mathrm{n}_{\mathrm{e}} \mathrm{V}_{\mathrm{e}}$ is simply the flux of beam electrons onto the foil. $\mathrm{E}$ is $3.41 \times 10^{-13} \mathrm{~J} / \mathrm{cm}$ [1] for Kapton, $8.4 \times 10^{-13} \mathrm{~J} / \mathrm{cm}$ for frosted quartz [2], and is the energy loss of each incident electron per length of foil traversed. $c_{p}$ is the heat capacity. The heat capacity is equaled to $1.55 \mathrm{~J} / \mathrm{cm}^{3}-\mathrm{K}$ [3] for Kapton and $2 \mathrm{~J} / \mathrm{cm}^{3}-\mathrm{K}$ [1] for frosted quartz; in both of these cases the values are assumed to be independent of temperature for simplicity. $r$ indicates position on the foil.

For a $3 \mathrm{kA}$ FXR beam with flat current profile normally incident on an OTR foil, Jacob [1] gives minimum tolerable spot sizes for different foil materials before melting and significant damage. If the uniform beam radius is $1 \mathrm{~cm}$, and no heat loss or transport occurs after each pulse, the temperature on the foil would raise $\sim 100-200 \mathrm{~K}$ for each pulse depending on foil material; this raise is independent of the foil thickness since the rate of energy deposition per thickness is a constant. In effect, even at moderately large beam radii, the foils would quickly be destroyed after 5-10 shots. Hence, some understanding of the primary foil cooling mechanism is required to model temperature dependent OTR effects and to prevent damage to the foils. Note that a foil at 45 degrees would result in a larger effective foil area shaped like an ellipse and lower overall 
temperatures. The elliptical problem is more difficult to approach analytically and remains future work.

To this end, the first task involved modeling efforts which confirmed that the typically low thermal conductivity of the OTR foils results in heat transfer that is insufficient to cool the foils back down to reasonable temperatures in the minute or so interval between shots. For a given initial temperature profile, $\mathrm{T}_{\mathrm{i}}(\mathrm{r})$, after a shot, the 1-D radial heat conduction equation can be analytically solved using Bessel-Fourier series to give for the foil temperature as a function of radius and post-shot time:

$$
T(r, t)=\sum_{m=1}^{\infty} \frac{2 J_{0}\left(k_{m} r\right) e^{-k_{m}^{2} c^{2} t}}{R^{2} J_{1}^{2}\left(\alpha_{m}\right)} \int_{0}^{R} r T_{i}(r) J_{0}\left(k_{m} r\right) d r
$$

where $\mathrm{R}, \sim 10 \mathrm{~cm}$, is the foil boundary and held at room temperature in the model, $\alpha_{\mathrm{m}}$ the zeros of the zeroth order $\mathrm{J}_{0}$ Bessel function, $\mathrm{k}_{\mathrm{m}}$ is equal to $\alpha_{\mathrm{m}} / \mathrm{R}$, and $\mathrm{c}^{2}$ is the thermal diffusivity and equal to $\mathrm{K} / \mathrm{c}_{\mathrm{p}}$ where $\mathrm{K}$ is the thermal conductivity of the material and can be dependent on temperature.

Figure 2.1 illustrates some results from Equation 2.2 for Kapton foils. Here, a constant thermal conductivity value of $\sim 0.004 \mathrm{~W} / \mathrm{cm}-\mathrm{K}$ is assumed from [4] as the VN type Kapton employed is suppose to be similar to the HN type except with superior “dimensional stability"[3]. This conductivity is for $\mathrm{HN}$ temperatures greater than $\sim 320 \mathrm{~K}$ and is estimated based on the fit in Figure 2 of [4]. Other Kapton film types, such as H, have lower conductivities. Clearly, the peak temperatures lower only slightly during the minute or so in-between the typical FXR shot. Hence, other mechanisms, such as radiation cooling, must be lowering the temperatures also. This can be quickly estimated; the heat loss per area for a hot blackbody foil to its surroundings is simply $\mathrm{Q}=2 \sigma\left(\mathrm{T}^{4}-\mathrm{T}_{0}^{4}\right)$, where $\sigma$ is the Stefan-Boltzmann constant and $T_{0}$ is the surrounding temperature, taken as $20 \mathrm{C}$. The factor of 2 comes from the fact that both sides of the foil can radiate. At $\mathrm{T}=400 \mathrm{C}, \mathrm{Q} \sim 2.2 \mathrm{~W} / \mathrm{cm}^{2}$. For a $0.013 \mathrm{~cm}$ thick $(5 \mathrm{mils})$ Kapton foil, the thermal heat capacity per foil area and Kelvin is only $\sim 0.02 \mathrm{~J} / \mathrm{cm}^{2}-\mathrm{K}$. This gives a total energy of only $\sim 10 \mathrm{~J} / \mathrm{cm}^{2}$ for a foil heated from room temperature to $400 \mathrm{C}$. Thus, radiation cooling can 
work quickly to decrease peak foil temperatures assuming reasonable values of thermal emissivity.

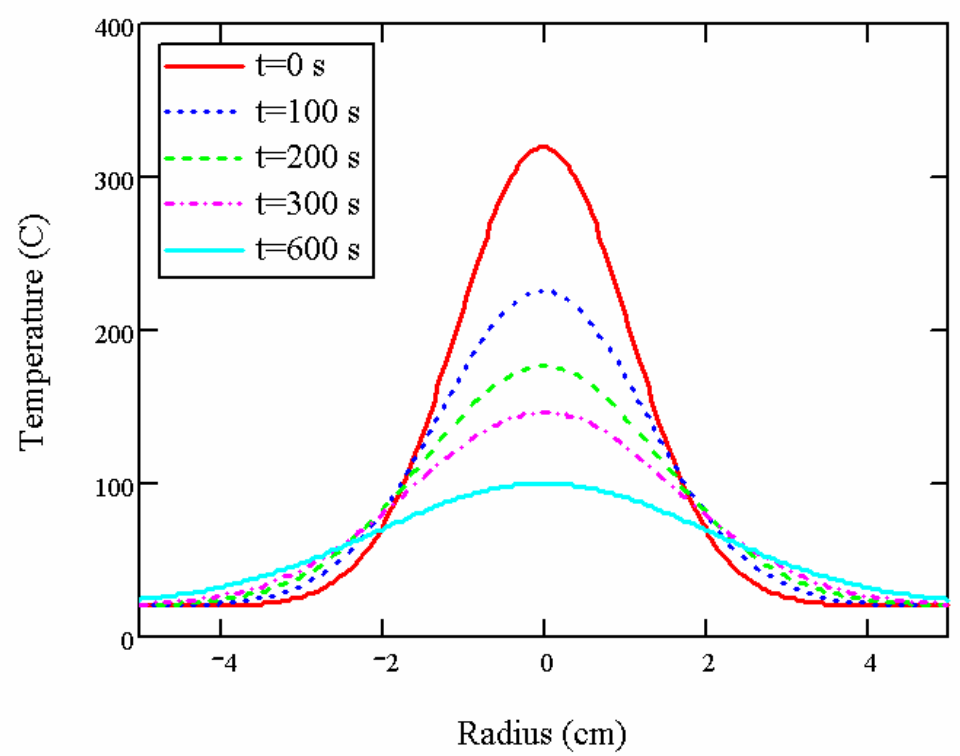

Figure 2.1: Kapton temperature profiles as a function of cooling time assuming conductive cooling only. The initial profile at $\mathrm{t}=0 \mathrm{~s}$ is based on a Gaussian beam current heating profile but does not correspond to a specific FXR shot.

Having identified thermal radiation as the primary mechanism for foil cooling in-between shots, the effect of radiation cooling and temperature build-up in a shot series was studied by assuming an uniformed beam profile and using a semi-analytical solution approach. Specifically, an analytical solution for the time it takes for a foil to cool by radiation from a specified temperature, $T_{i}$, to a final temperature, $T_{f}$, was found and given by:

$$
\Delta t_{c}=-\alpha \int_{T_{i}}^{T_{f}} \frac{d T}{\left(T^{4}-T_{0}^{4}\right)}=-\frac{\alpha}{2 T_{0}^{3}}\left[\frac{1}{2} \ln \left(\frac{T_{f}-T_{0}}{T_{f}+T_{0}}\right)-\tan ^{-1}\left(\frac{T_{f}}{T_{0}}\right)-\frac{1}{2} \ln \left(\frac{T_{i}-T_{0}}{T_{i}+T_{0}}\right)+\tan ^{-1}\left(\frac{T_{i}}{T_{0}}\right)\right]
$$

where $\alpha=\mathrm{c}_{\mathrm{p}} \mathrm{L} / \varepsilon_{\mathrm{T}} \sigma \mathrm{F}, \mathrm{L}$ is the thickness of the foil, $\varepsilon_{\mathrm{T}}$ the emissivity of the foil, and $\mathrm{F}$ the number of sides the foil can radiate from. For the FXR Kapton and frosted quartz OTR foils, a $\varepsilon_{\mathrm{T}} \sim 0.75$ is used; some data is available from [5-6]. L is $0.013 \mathrm{~cm}$ for Kapton and $0.038 \mathrm{~cm}$ for frosted quartz, and $\mathrm{F}$ is 2 . F can be less than 2, for example, in cases where the foil is covered with different coatings that have very low emissivity. The surroundings are assumed to be black and held at $\mathrm{T}_{0}=293 \mathrm{~K}$. Note that for radiation 
cooling, thinner foils are better since they have less stored energy but no less radiating surface area.

Using Equation 2.3, a Mathcad program utilizing a root finder was created to estimate foil temperatures as a function of shot number and cooling period in-between shots. Figure 2.2 plots the frosted quartz case for an FXR beam with a radius of $1 \mathrm{~cm}$. A steady-state temperature is quickly reached after several shots for the examined parameters. Because of the thinness of the Kapton, the radiation model predicts that they return to near room temperature very rapidly in-between shots.

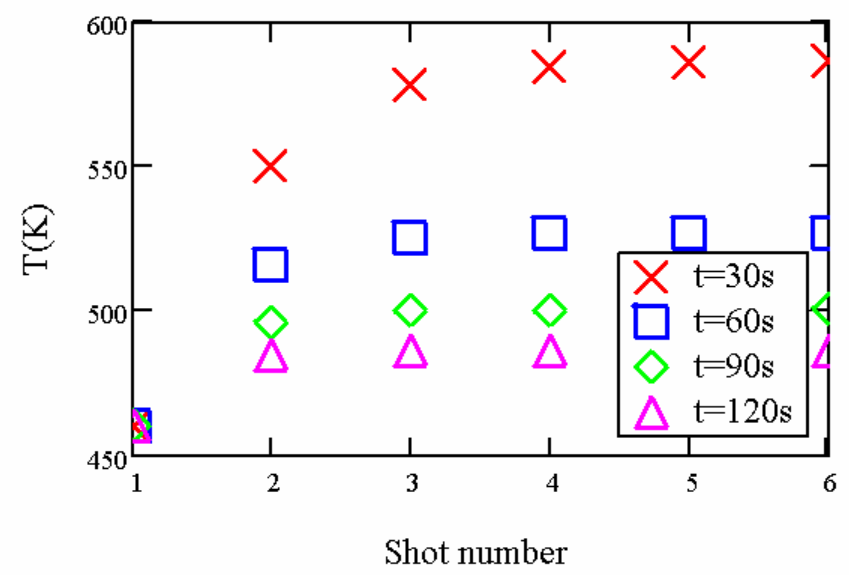

Figure 2.2: Post shot temperatures of a $0.038 \mathrm{~cm}$ thick frosted quartz foil for uniform FXR beams of $1 \mathrm{~cm}$ radius assuming radiation losses only and a thermal emissivity of 0.75. Each curve represents different cooling periods in between shots.

In summary, the primary OTR foil cooling mechanism in-between FXR shots was identified to be thermal radiation. This conclusion stems from foil temperature estimates made based on conduction and radiation cooling; the survivability of the foils also suggests relatively rapid cooling in-between shots. Modeling of the foil temperatures as a function of shot number in a series of repeat shots and cooling intervals found that in most cases, a steady-state post shot foil temperature is reached after very few shots. This model can be used to prevent permanent damage to OTR foils and for use in temperature dependent OTR emission models as long as detailed profiles are not needed. Significantly more complex thermal modeling incorporating temperature dependent heat capacity, thermal conduction, thermal emissivity, and detailed beam and foil geometric 
LLNL-TR-402621

parameters might be required if very accurate temperature profiles of the foils are needed to model the OTR emission.

Section IIb: Kapton heating experiments in He furnace

A preliminary series of experiments was performed on the Kapton material used for the FXR OTR foils. Specifically, clean Kapton foils were taken up to specified temperatures in a helium furnace and kept at temperature for $\sim 10$ minutes. A He furnace was used to prevent chemical reactions with the Kapton. The purpose of this test was to determine visual changes in the Kapton as it approached its decomposition temperature, typically around $\sim 600 \mathrm{C}$ and compare them to changes in beam damaged Kapton. An example of a beam damaged Kapton foil is given in Figure 2.3. A glass-phase transition is supposed to occur before Kapton decomposition [3]. If the phase transition energy of Kapton is low compared with the beam deposition energy and the foil does not cool in-between shots, the FXR OTR foils should exhibit some of the same visual changes as the foils in the furnace experiment. Thus, the absence of similar visual changes on the FXR OTR Kapton foils would support the conclusion that the foils are cooling relatively rapidly inbetween shots. This test is not exact but was done to provide useful qualitative information. Figure 2.4 gives the results. Physically, the material darkened with increasing temperature and became slightly more brittle. At $600 \mathrm{C}$, the material fractured easily. Clearly, the OTR foil in Figure 2.3 does not show any broad color gradients suggesting the type of changes that occurred in Figure 2.4 which might have been caused by large beams. In fact, the small beam damaged area in Figure 2.3 became silver or light orange in color and glossy (tear-shaped area), with the exception of the black spot which could indicate changes caused by very intense beam heating. In any event, the furnace test suggests that the phase transition energy is large compared with the beam deposition energy, and, or that for most cases, the foil does cool and even out temperature gradients quickly to prevent damage. The silver areas in the damage foil could indicate a damage mechanism specific to beam-target interactions instead of a temperature effect. 


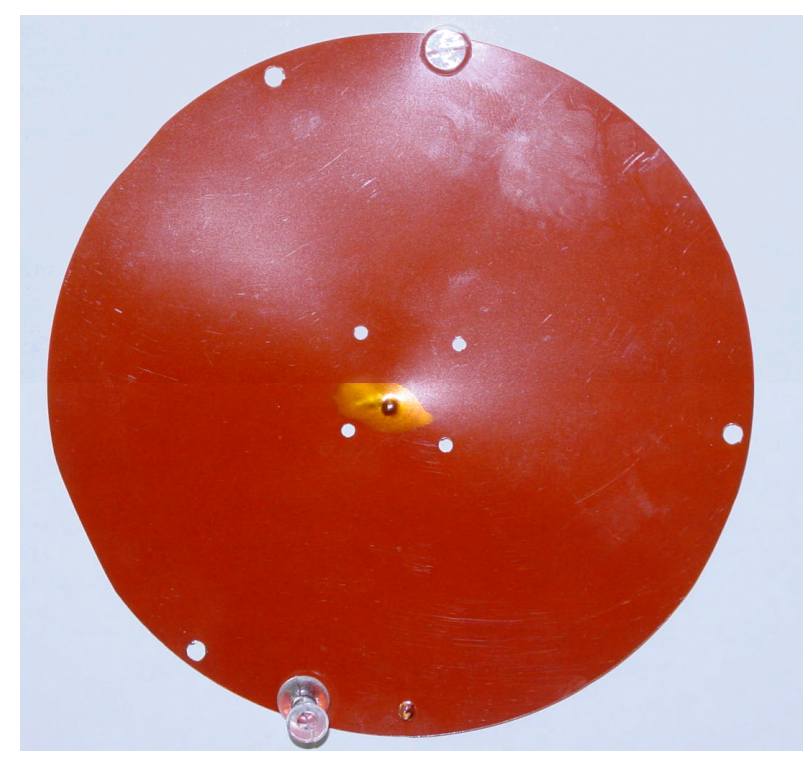

Figure 2.3: A damaged FXR Kapton OTR foil. The black spot is likely from a beam of similar radius. The center fiducial holes form a $\sim 2 \mathrm{~cm} \times 2 \mathrm{~cm}$ square.

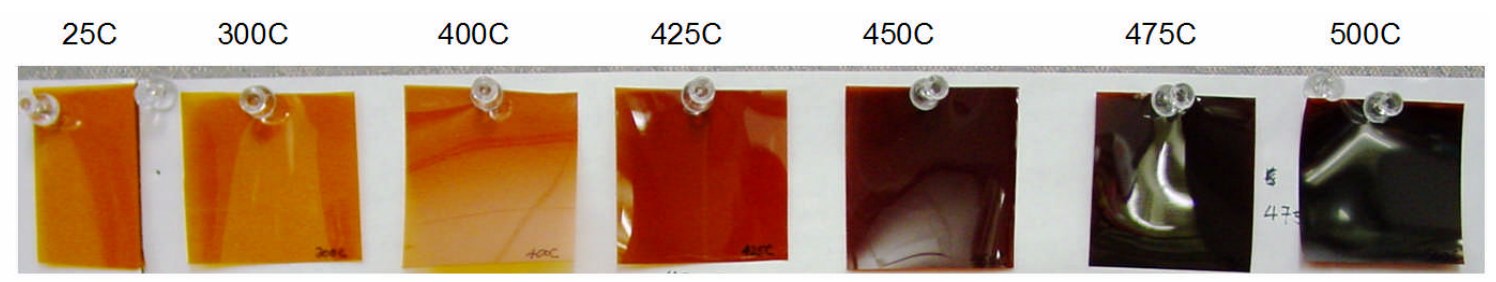

Figure 2.4: Kapton pieces from the He furnace test. The sample at $600 \mathrm{C}$ fractured and is not shown. Its color was darker than the $500 \mathrm{C}$ case.

\section{Section II References}

[1] J.S. Jacob et al., UCRL-TR-214037, LLNL Technical Report, 2005.

[2 See for example, pgs. 44-45 in G.F. Knoll, "Radiation Detection and Measurement", John Wiley \& Sons, $2^{\text {nd }}$. Ed, 1989.

[3] DuPont Company, "Kapton HN Technical Data Sheet”, www.dupont.com

[4] D.L. Rule, D.R. Smith, and L.L. Sparks, Cryogenics, 36, 283-290, 1996.

[5] E.J. Simburger et al., IEEE Photovoltaic Spec. Conf., 1053-1056, 2000. Table 2.

[6] V.V. Pukhonin and A.S. Chaikin, Zhurnal Prikladnoi Spektroskopii, 16, 1, 138-142, 1972. (Available in English via PPC Consultants Bureau, UDC 543.42, 1974) 


\section{Section III: OTR diagnostic experimental results}

During this fiscal year, several sets of FXR experiments were done to investigate temperature dependent issues related to the FXR dielectric and metallic foil OTR diagnostic in addition to examining shot repeatability on FXR. The basic premise behind these experiments was to utilize similar series of repeat shots to prove temperature dependent effects from dielectric foil OTR. Using repeats in theory isolates several OTR specific issues in comparison with the previous efforts where the OTR data were collected over current and timing scans with different time intervals and shot order. Specifically both effects from instantaneous foil heating and shot-to-shot base or prebeam foil temperature variations can be studied. The results would ultimately allow benchmarking of a comprehensive simulation or synthetic diagnostic which could then provide a correction between spot RMS radius and real beam RMS radius.

The proposed experiments simply entailed running a series of the same moderately tight beam onto Kapton, frosted quartz, and Al coated quartz foils at the FXR diagnostic cross with a constant cooling time between shots. The spot-size from the Al coated quartz foil should be constant for such an experiment since in theory metallic foils are immune to temperature based OTR changes, while both the Kapton and frosted quartz should see some shot-to-shot changes in the spot-size until a steady-state temperature is reached. The Kapton based spot should go to a steady-state almost right away since the thin Kapton foils should reach a steady-state temperature profile rapidly compared with frosted quartz. The frosted quartz might take up to four shots depending on the beam radius and cooling time. Also, shots with foils at large angle to the beam should reach their steady-state temperature profiles quicker since they effectively spread the beam over a larger foil surface as discussed in the last section.

Assuming perfect repeat shots and several shots before steady-state temperatures are reached, it is expected that the spot RMS radius will increase with shot number if the permittivity of the foil decreases with temperature; the total counts should decrease with shot number and hence spot RMS radius. The spot RMS radius should increase with shot 


\section{LLNL-TR-402621}

number since the center of the foil should progressively emit less OTR as it gets hotter from shot-to-shot, resulting in a broader but less intense OTR spot, and the total counts should decrease with shot number since the foil temperature overall increases with shot number until it reaches steady-state. Thus in this case, for a set of perfect repeats, the total counts decreases with spot RMS radius, counter to the trends and Houck's model (which considered instantaneous heating only or the same starting foil temperature for each shot) in Section I. This means that temperature effects based on instantaneous heating is typically dominant. If the permittivity increases with temperature, the total counts would decrease with spot RMS radius for a set of perfect repeats, again opposite to the trends in Section I. Table 3.1 provides a summary of the expected total counts and radius trends for different types of shot series, assuming the beam center does not move shot-to-shot.

\begin{tabular}{|c|c|c|c|}
\hline For $\varepsilon \downarrow$ when Temp $\uparrow$ & $\begin{array}{l}\text { Total counts with } \\
\text { spot RMS radius }\end{array}$ & $\begin{array}{l}\text { Total counts with shot } \\
\text { number }\end{array}$ & $\begin{array}{l}\text { Spot RMS radius with } \\
\text { shot number }\end{array}$ \\
\hline $\begin{array}{l}\text { Instantaneous beam heating } \\
\text { temperature effects only and } \\
\text { beam repeats perfectly }\end{array}$ & $\begin{array}{l}\text { No trend, only one } \\
\text { spot radius and } \\
\text { same total counts }\end{array}$ & $\begin{array}{l}\text { No change in total } \\
\text { counts }\end{array}$ & No change in spot radius \\
\hline $\begin{array}{l}\text { Instantaneous beam heating } \\
\text { temperature effects only and } \\
\text { beam does not repeat }\end{array}$ & $\uparrow$ & $\begin{array}{l}\text { Depends on real beam } \\
\text { radius for each shot }\end{array}$ & $\begin{array}{l}\text { Depends on real beam } \\
\text { radius for each shot }\end{array}$ \\
\hline $\begin{array}{l}\text { Shot-to-shot temperature } \\
\text { effects only and beam repeats } \\
\text { perfectly }\end{array}$ & $\downarrow$ & $\downarrow$ & $\uparrow \uparrow$ \\
\hline $\begin{array}{l}\text { Shot-to-shot temperature } \\
\text { effects only and beam does } \\
\text { not repeat }\end{array}$ & $\begin{array}{l}\text { Depends on shot } \\
\text { history }\end{array}$ & $\begin{array}{l}\text { Depends on shot history } \\
\text { and current shot }\end{array}$ & $\begin{array}{l}\text { Spot radius } \\
\text { progressively get larger } \\
\text { than real beam radius } \\
\text { for each shot }\end{array}$ \\
\hline \multicolumn{4}{|l|}{ For $\varepsilon \uparrow$ when Temp $\uparrow$} \\
\hline $\begin{array}{l}\text { Instantaneous beam heating } \\
\text { temperature effects only and } \\
\text { beam repeats perfectly }\end{array}$ & $\begin{array}{l}\text { No trend, only one } \\
\text { spot radius and } \\
\text { same total counts }\end{array}$ & $\begin{array}{l}\text { No change in total } \\
\text { counts }\end{array}$ & No change in spot radius \\
\hline $\begin{array}{l}\text { Instantaneous beam heating } \\
\text { temperature effects only and } \\
\text { beam does not repeat }\end{array}$ & $\downarrow$ & $\begin{array}{l}\text { Depends on real beam } \\
\text { radius for each shot }\end{array}$ & $\begin{array}{l}\text { Depends on real beam } \\
\text { radius for each shot }\end{array}$ \\
\hline $\begin{array}{l}\text { Shot-to-shot temperature } \\
\text { effects only and beam repeats } \\
\text { perfectly }\end{array}$ & $\downarrow$ & $\uparrow$ & $\downarrow$ \\
\hline $\begin{array}{l}\text { Shot-to-shot temperature } \\
\text { effects only and beam does } \\
\text { not repeat }\end{array}$ & $\begin{array}{l}\text { Depends on shot } \\
\text { history }\end{array}$ & $\begin{array}{l}\text { Depends on shot history } \\
\text { and current shot }\end{array}$ & $\begin{array}{l}\text { Spot radius } \\
\text { progressively get } \\
\text { smaller than real beam } \\
\text { radius for each shot }\end{array}$ \\
\hline
\end{tabular}


In this way shot-to-shot type temperature effects have a distinct marker in a series of repeated shots with proper beam parameters and cooling intervals, and if significant would reveal themselves. Another way to see this is that perfect repeat shots apply the same instantaneous heating profile to different pre-beam foil temperature profiles during the shot series (the significant geometric factor discussed in Section IV is not temperature dependent and hence does not affect the test discussed here). Figure 3.0 illustrates qualitatively the spot RMS radius trends for a series of repeat shots with significant prebeam foil temperature profiles using Houck's preliminary foil model which assumes the OTR emission varies with the permittivity $\varepsilon$, as $|1-\varepsilon|^{2}$. On a side note, if complete isolation of the instantaneous heating effects is desired, the cooling interval or time in between shots could simply be increased to approximately five minutes, based on the calculations in Section II, to ensure the foil is totally cooled.

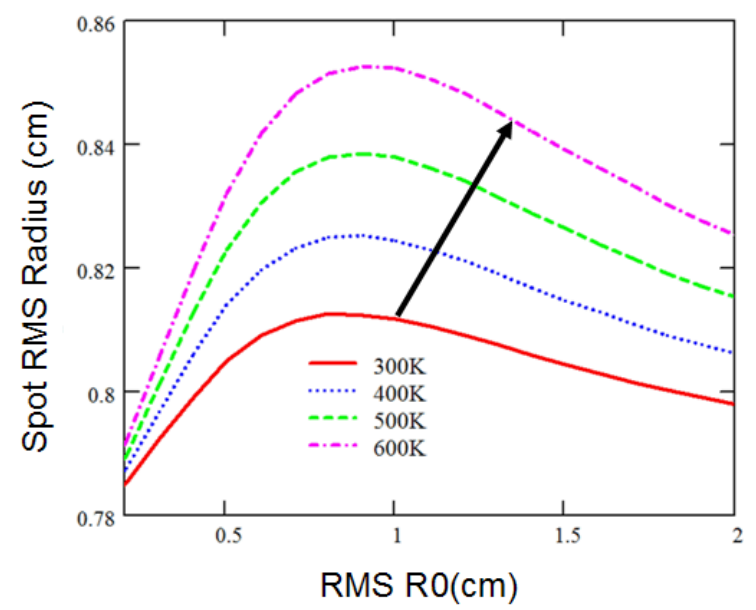

Figure 3.0: Sample OTR spot radius for an FXR beam with RMS radius of $0.75 \mathrm{~cm}$ as a function of foils with different sample base (e.g. pre-beam) Gaussian temperature profiles assuming an isotropic OTR emission varying with $|1-\varepsilon|^{2}$. R0 is the RMS radius of the Gaussian and the temperatures given are the peaks. In this case a trial permittivity varying approximately from 2 to 3 and decreasing with temperature was assumed. The black arrow indicates qualitatively the spot RMS radius path for a series of repeat shots.

If shot-to-shot based temperature effects are not important (because of rapid cooling, for example) or a steady-state temperature profile is reached within one shot, there should be approximately no variation in either the spot RMS radius or total counts. Also, if the shots do not repeat properly and shot-to-shot temperature effects are insignificant compared with the effects from instantaneous beam heating, then the data should revert to 
the total counts trends found by Houck and given in Section I; namely the total counts should increase with spot RMS radius for a permittivity that decreases with temperature. Shot-to-shot temperature effects should not dominate over the instantaneous beam heating based effects as long as the instantaneous heating of the foil results in significantly higher temperatures than the initial pre-beam foil temperatures. This is typically the case considering the temperature modeling in Section II.

Isolating shot-to-shot based temperature effects can hence be difficult, but because of the expected distinct trends associated with them in these repeat experiments compared with temperature effects from instantaneous heating, should be obvious if their isolation was successful. Fortunately, even if these ideal experimental conditions are not met, the repeat shots will nevertheless provide ample data for modeling the dielectric foil OTR diagnostic. Note that the real beam radius does not even necessarily have to be determined from the OTR data in order to see temperature dependent effects if shot reproducibility is good.

Thus, the proposed series of experiments in theory allows us to test FXR shot reproducibility along with providing information on temperature based OTR effects from instantaneous beam heating and shot-to-shot base foil temperature build-ups. In the end, if shot repeatability is good across the test series and other effects such as backstreaming ions are minimum, then the spot RMS radii data can be used to benchmark OTR simulations, confirm temperature based OTR issues, and thus would allow the simulation or synthetic diagnostic be used to accurately interpret spot-size images from the FXR OTR diagnostics operating with dielectric foils.

In other words, ideally, accurate modeling and understanding of OTR from dielectric foils would be confirmed if the results, specifically the spot RMS radius and total counts from the three different repeat series, could be reconciled shot-to-shot in each series after accounting for temperature based effects, and then respectively with the Al benchmark. This is independent of the qualitative trends that would be available from the data even without these detailed benchmarking. 
In this Section, the results so far from the above repeat series on four different experimental days are examined and preliminarily discussed. The first day involved experiments with the frosted quartz foil at nominally 45 and 75 degrees, followed by experiments with a Kapton foil on the second day. The third and fourth days used Al covered quartz foils. The data set is still under detailed analysis; there is supporting evidence for some temperature based effects but also numerous anomalies.

For the quartz data, the spot RMS radius declined with shot number but the total counts increased with spot RMS radius and hence decreased with shot number. Therefore shotto-shot based temperature effects were not isolated, and the beam did not repeat based on the trends in Table 3.1. These were the first and second series of the run day. The trends do support temperature dependent effects from instantaneous beam heating, however. Also, additional evidence suggesting beam repeatability problems is the total variation in spot RMS radius as a function of shot number; it is too large to be solely caused by any temperature based OTR effects. The radius and total counts trend follows Houck's temperature dependent OTR model if the permittivity in the OTR frequency range for quartz decreased with foil temperature. A literature search is ongoing for the permittivity data.

The Kapton data were limited due to foil heating damage near a fiducial hole, but interesting phenomena, including some doughnut like spot-size images, were observed. Under certain assumptions, the synthetic diagnostic simulations in Section IV can produce these hollow spot profiles. The limited amount of Kapton data suggest that a steady-state temperature profile and RMS radius was reached quickly, as would be expected from modeling results described in Section II. The RMS radii, although suspect, are consistently below the RMS radii from the quartz foils.

All the first shots for the dielectric foil runs examined so far in a series gave abnormally high total counts with a correspondingly large radius; this also happened for a significantly long break in-between shots. 
The Al coated foil benchmarking data were also revealing; the troubling aspect is that the spot RMS radius on the $\mathrm{Al}$ covered foil experiments that occurred as the first series of the day declined with shot number. Shot reproducibility issues on FXR when warm-up shots are not used is the most likely explanation as long as the assumption that metal foils are immune to temperature based effects is correct. This supports the reproducibility issues inferred from the quartz data. Testing with a second series of repeated shots using an Al covered quartz foil that was only inserted in the middle of the series provided evidence that this was a shot repeatability issue and not temperature based. Thus, again the quartz and Kapton data tests above would not have been optimum as some of those shots likely had noticeable repeatability issues. These repeatability concerns could be partly the reason why previous attempts to compare beam radii with simulations proved challenging.

Lastly, more potential issues for dielectric foils were identified this year during analysis of these experimental data. For example, a related and potential problem unexamined so far for the FXR OTR diagnostic is that the foil itself, independent of material, might be significantly perturbative due to back-streaming ions; variations in the spot size and total counts as a function of shot number might stem partially from the non-steady state foil cleaning that occurs during each repeat shot. However, at the $\sim 10^{-6}$ torr pressures that FXR runs at, monolayer formation times should be significantly shorter than the typical minute intervals between shots. Additional effects such as dielectric foil charging have also not been studied and would be difficult to quantify.

Detailed synthetic diagnostic simulations of the FXR OTR diagnostic are discussed in Section IV and work is in progress to apply those simulations to the data sets in this Section. 
Section IIIa: Frosted quartz foil experiments on Dec. $8^{\text {th }}, 2006$.

On this day, two series of repeat shots were done. The settings used was a Rex06 tune with DR1\&2 at $350 \mathrm{~A}$ and L43\&44 at 300 A. The first series, spanning shots 158243158252 and dubbed Q45R1 here, were the first shots in the morning with no prior warmup shots. A set of 10 repeated shots were done with one minute cooling intervals except for the interval between the first and second shot, which was $\sim 7$ minutes. The frosted foil was nominally at 45 degrees with respect to the beam but was in reality closer to 55 degrees. The beam impacted the frosted side of the foil and that side was imaged. A fstop of 22 was used which in retrospect might be too high since it resulted in a tight OTR acceptance solid angle and makes the data in general more susceptible to the geometric effects discussed in Section IV if the foil was not effectively frosted.

Figure 3.1-3.4 summaries the data set given by the post shot Matlab toolset discussed in Section I. Some of the data points had to be excluded in the Gaussian fit because of the fiducial holes. The spot RMS radius decreased with shot number, until the anomalous jump in the last shot. The total counts increased with the spot RMS radius and therefore decreased with shot number. Therefore shot-to-shot based temperature effects were not isolated, and the beam did not repeat based on the expected trends in Table 3.1. The trends do support temperature dependent effects from instantaneous beam heating, however. Also, additional evidence suggesting beam repeatability problems is the total variation in spot RMS radius as a function of shot number; it is too large to be solely caused by any temperature based OTR effects. The radius and total counts trend follows Houck's temperature dependent OTR model if the permittivity in the OTR frequency range for quartz decreased with foil temperature. A literature search is ongoing for the permittivity data. Lastly, Examination of the raw intensity data shows that the tighter spot RMS radii were caused by a real increase in centroid intensity, suggesting again non-repeatability and a real tightening of the beam during this series.

The results were not significantly changed by using different filter or background parameters in the toolset. There were no discernable trends in the examined background 
counts versus spot-size RMS radius. Plots of the displacement signals from the beam monitor after the diagnostic cross, given by Figures 3.5-3.6, do not reveal unique signatures explaining the jump in radius on the last shot. This was not unexpected since the centroid horizontal location on the OTR spot did not move too much during the series. It should be noted however that the last shot did have a similar spot centroid location to the first shot, which had a similar spot RMS radius.

The second series of experiments, spanning 158300-158311 and dubbed Q75R1 here, involved the frosted quartz foil at 75 degrees. The beam impacted the frosted side of the foil and that side was imaged. This was a 12 shot series since the first two shots were saturated at their peaks and the first four shots were actually $\sim 2-3$ minutes apart instead of the requested 1 minute interval. The gain was turned down to 160 from 200 after the first two shots. The same analyses were performed on this shot series; the data near the fiducial holes again were excluded form the fit, and luckily the saturated portions of the first two shots also lied in that region. The images were rotated by $\sim 3$ degrees in the analysis as they were tilted. The results are plotted in Figures 3.7-3.11. The trend of decreasing RMS radius with shot number is evident, but is less clear if the first four shots were dropped from the dataset due to saturation and timing irregularities. In contrast to the Q45R1 series, the data did reach a steady-state radius. Again, the total counts and radius trends indicate that shot-to-shot temperature effects were not isolated and also indicate some beam repeatability issues. However, the spot centroid during this series moved only slightly and, compared with the first series, the spot RMS radius appeared to be repeating to some extend after the first several shots.

Comparing the Q45R1 and Q75R1 series, the Q75R1 radii were noticeably lower than the Q45R1. This could be partly due to OTR geometric factors discussed in the Section IV. 

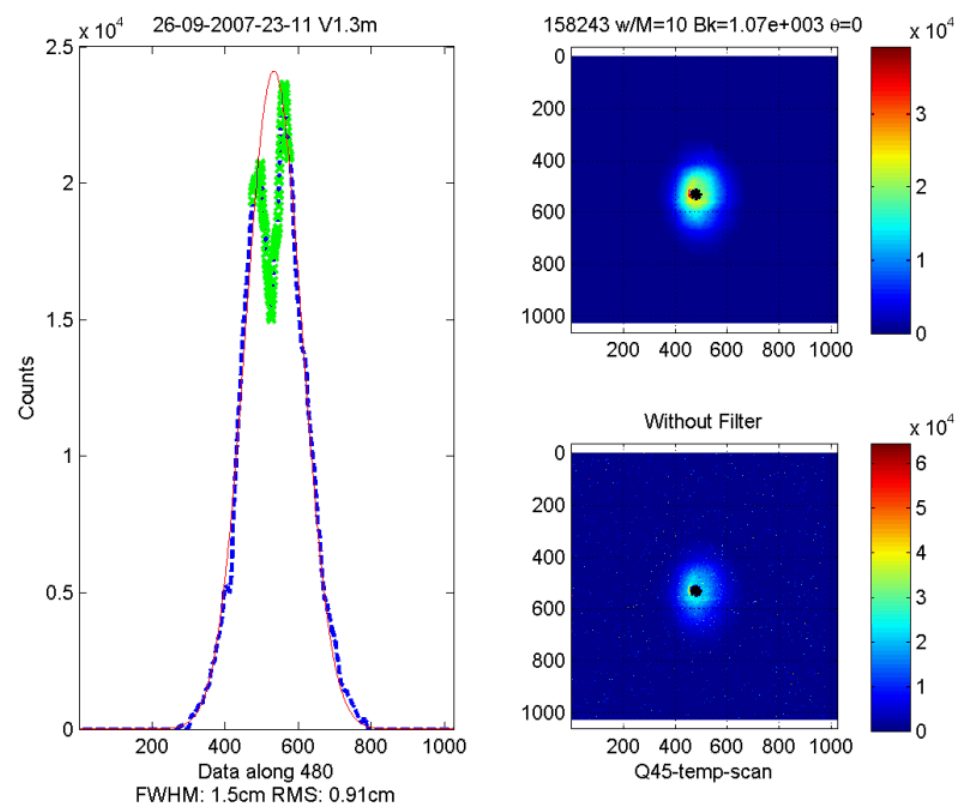

Figure 3.1: The first shot in the Q45R1 repeat experiment with a $\sim 55$ degree frosted quartz foil. The green points were excluded from the Gaussian fit which gave an RMS radius of $0.91 \mathrm{~cm}$.

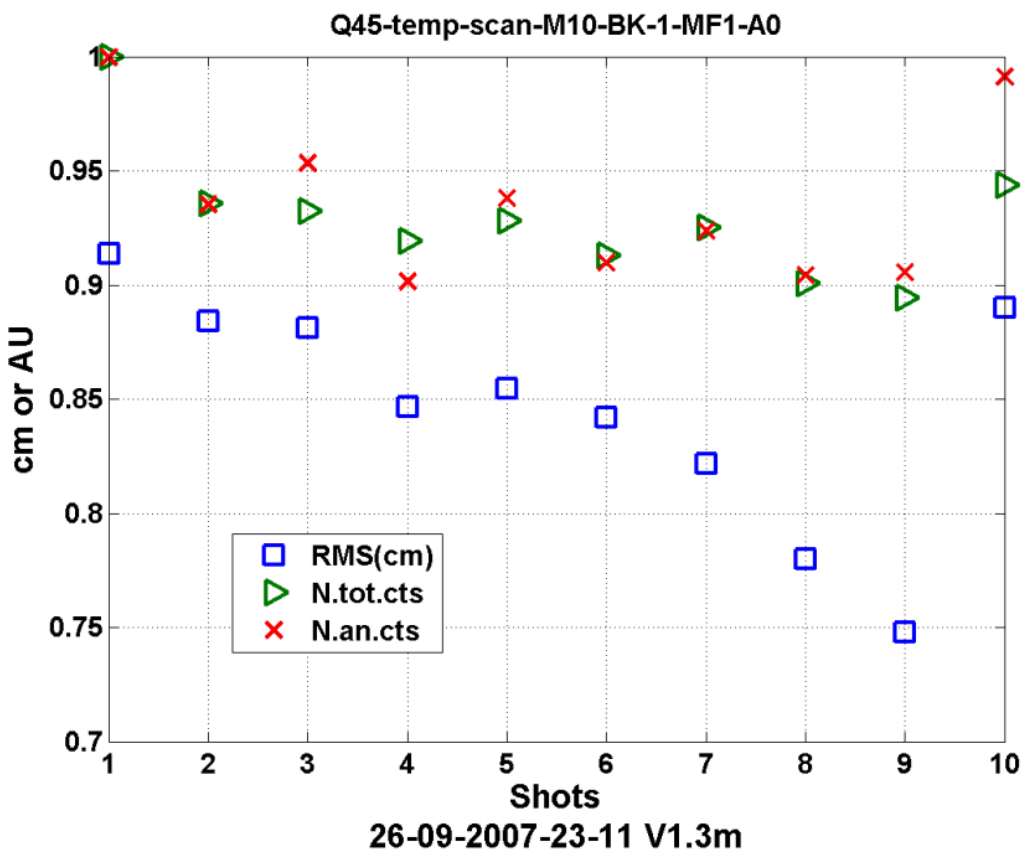

Figure 3.2: Summary of some key parameters in the Q45R1 repeat experiments. The RMS radius clearly decreased during the repeating shots until the jump on the last shot. The other data shown are the normalized total counts from the foil and the normalized calculated total counts based on the analytical Gaussian fit. The counts are normalized to the maximum counts in a series, in this case the first shot. 
LLNL-TR-402621

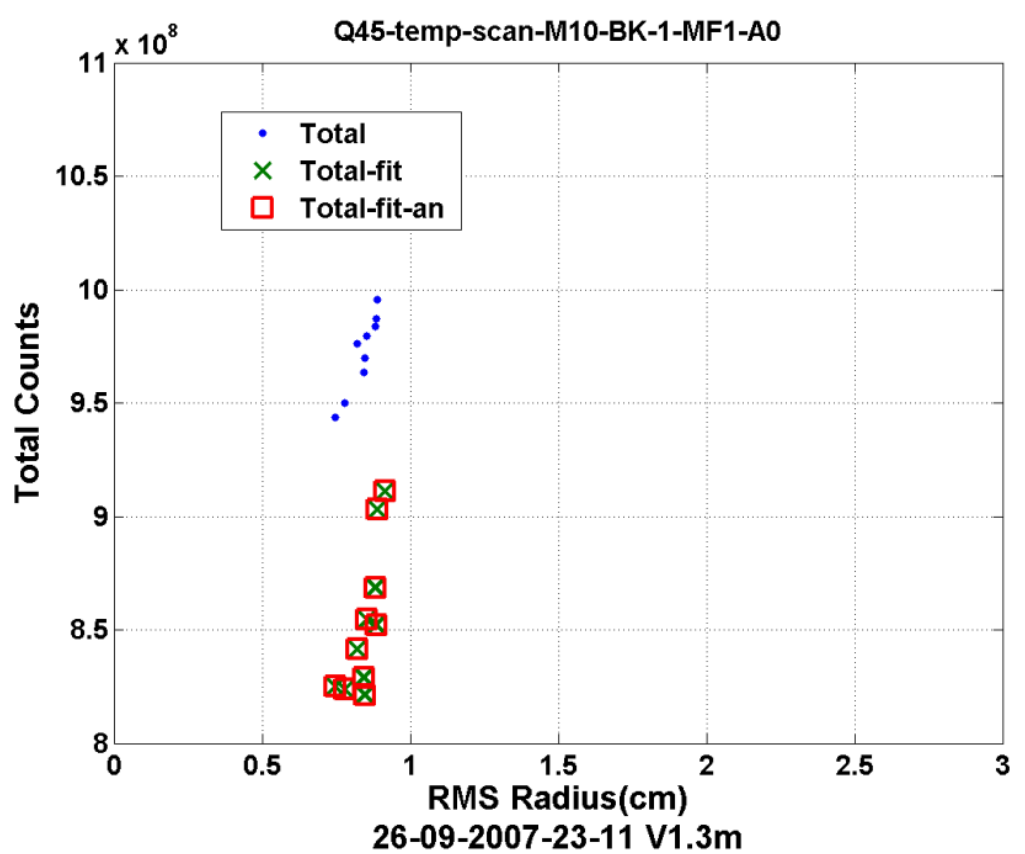

Figure 3.3: Total counts vs. RMS Radius for the Q45R1 repeat experiments. 'Total' indicates total counts from the foil after background subtraction, and 'Total-fit' indicates total counts calculated form the 1-D Gaussian fit of the data. ('Total-fit-an' is an internal check for 'Total-fit'). Clearly, both set of total counts increased with RMS radius.

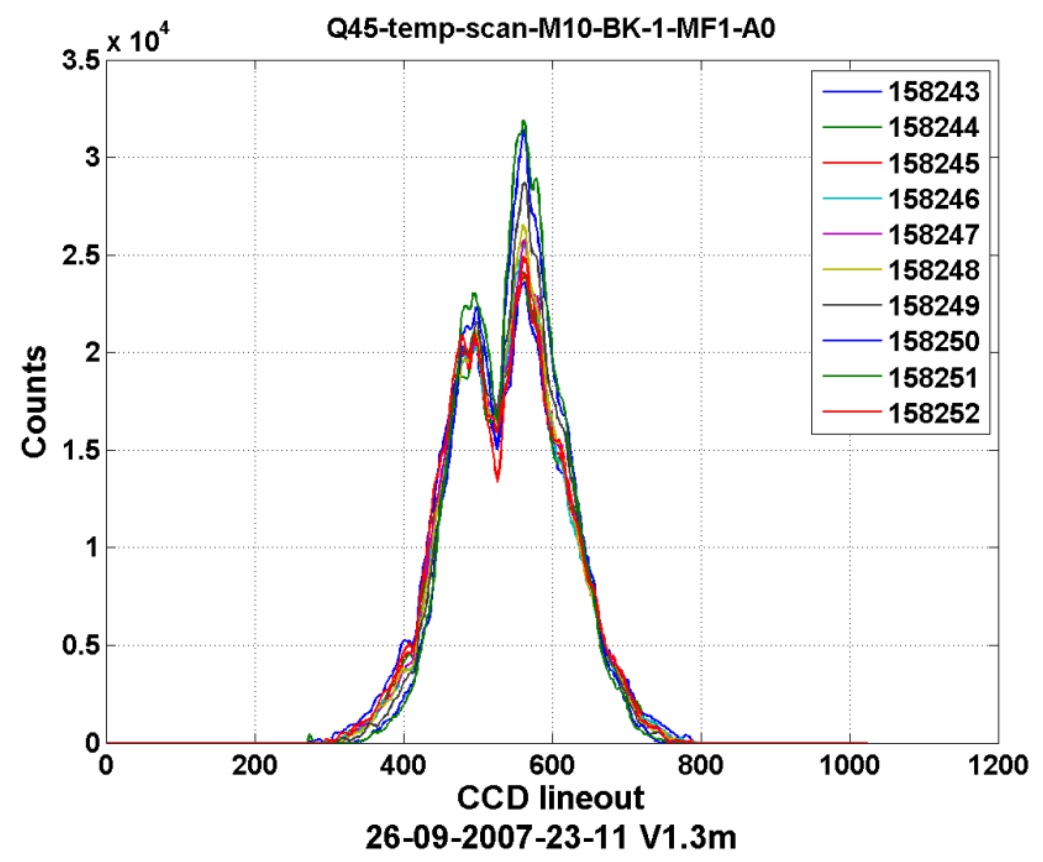

Figure 3.4: Summary of intensity data used to determine the RMS radius for the entire Q45R1 series. There is a general increase in peak intensity and tightening of the spotsize as the series progressed, resulting in smaller RMS radii until the last shot. 


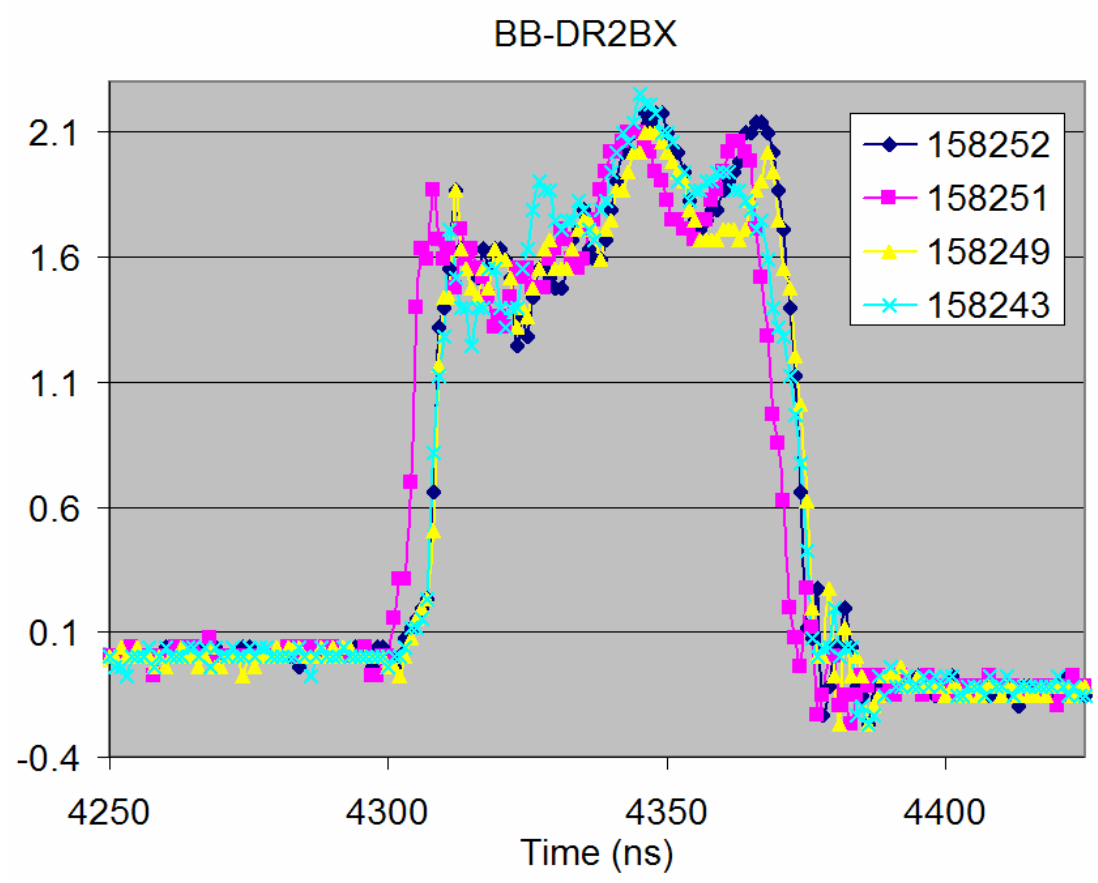

Figure 3.5: $\mathrm{x}$-displacement data from a probe after the diagnostic cross for some of the shots in the Q45R1 series. The camera triggered at $~ 4330$ ns. Shots 158252 and 158249 had noticeably different RMS radii but no difference in the displacement. The time base is relative and not absolute.

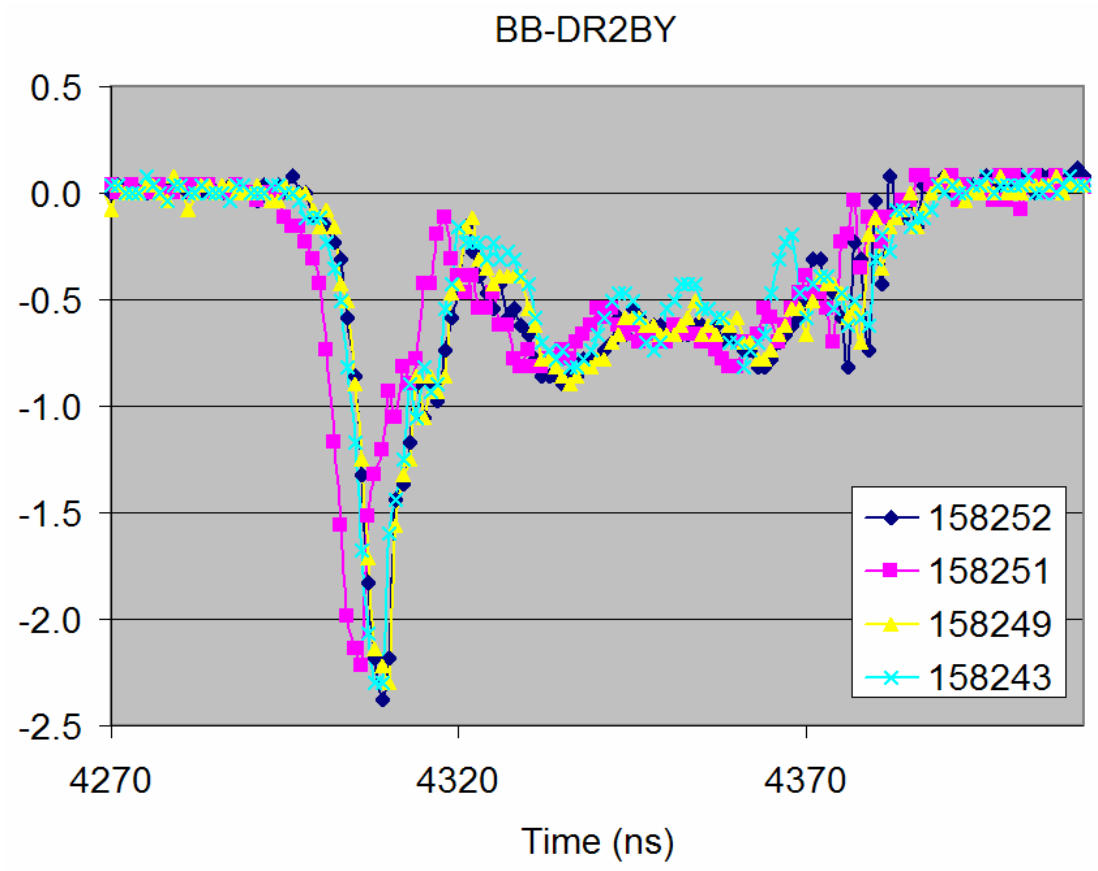

Figure 3.6: $y$-displacement data from a probe after the diagnostic cross for some of the shots in the Q45R1 series. The camera triggered at 4330 ns. Shots 158252 and 158249 had noticeably different RMS radii but no difference in the displacement. The time base is relative and not absolute. 

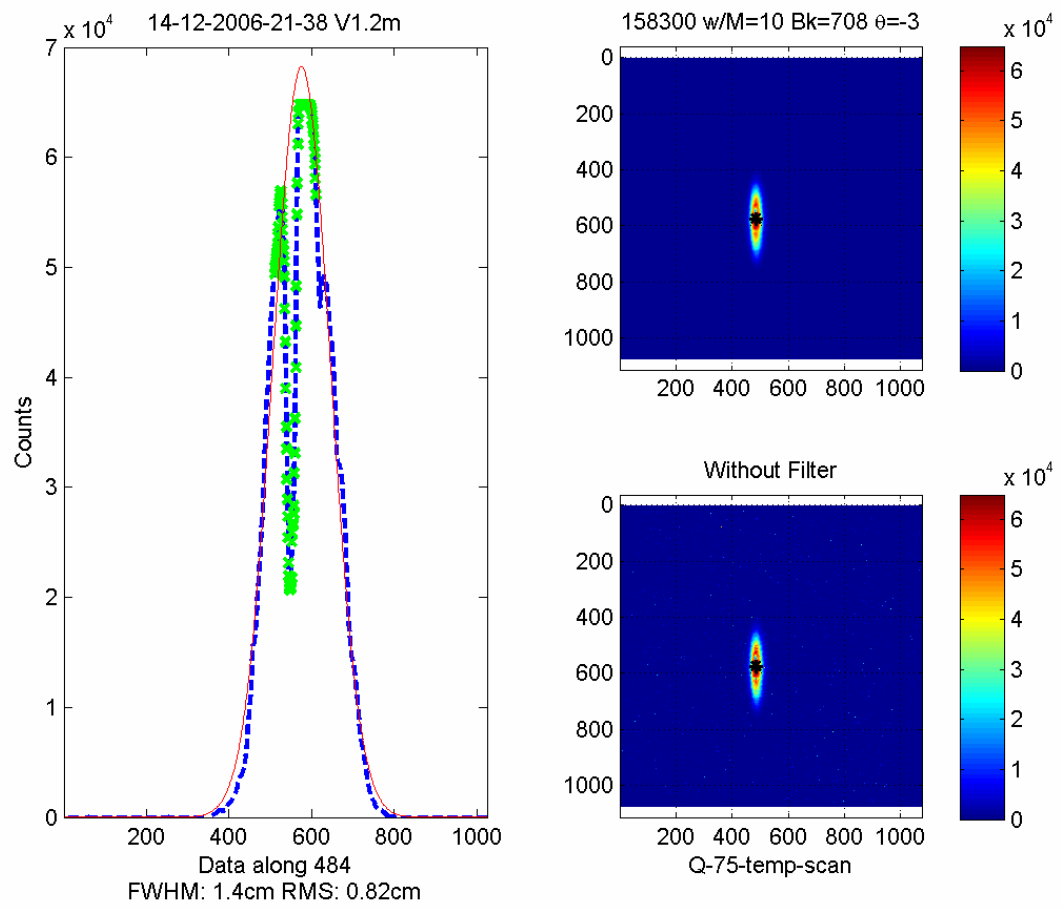

Figure 3.7: The first shot in the Q75R1 repeat experiment with a $\sim 75$ degree frosted quartz foil. The green points were excluded from the Gaussian fit which gave an RMS radius of $0.82 \mathrm{~cm}$. The image data was rotated 3 degrees before analysis.

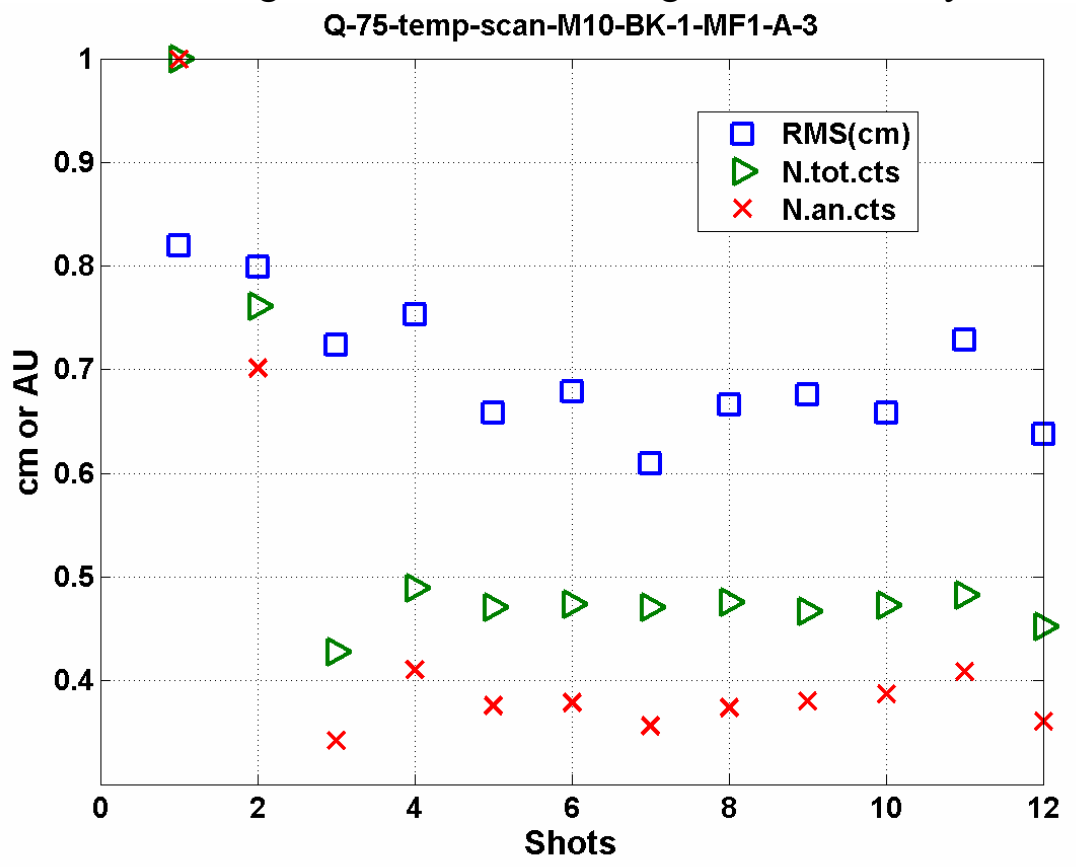

14-12-2006-21-38 V1.2m

Figure 3.8: Summary of some key parameters in the Q75R1 repeat experiments. The RMS radius decreased during the repeating shots. The other data shown are the normalized total counts from the foil and the normalized calculated total counts based on the analytical Gaussian fit. The normalization is based on the maximum total counts in the series, in this case the first shot. 
LLNL-TR-402621

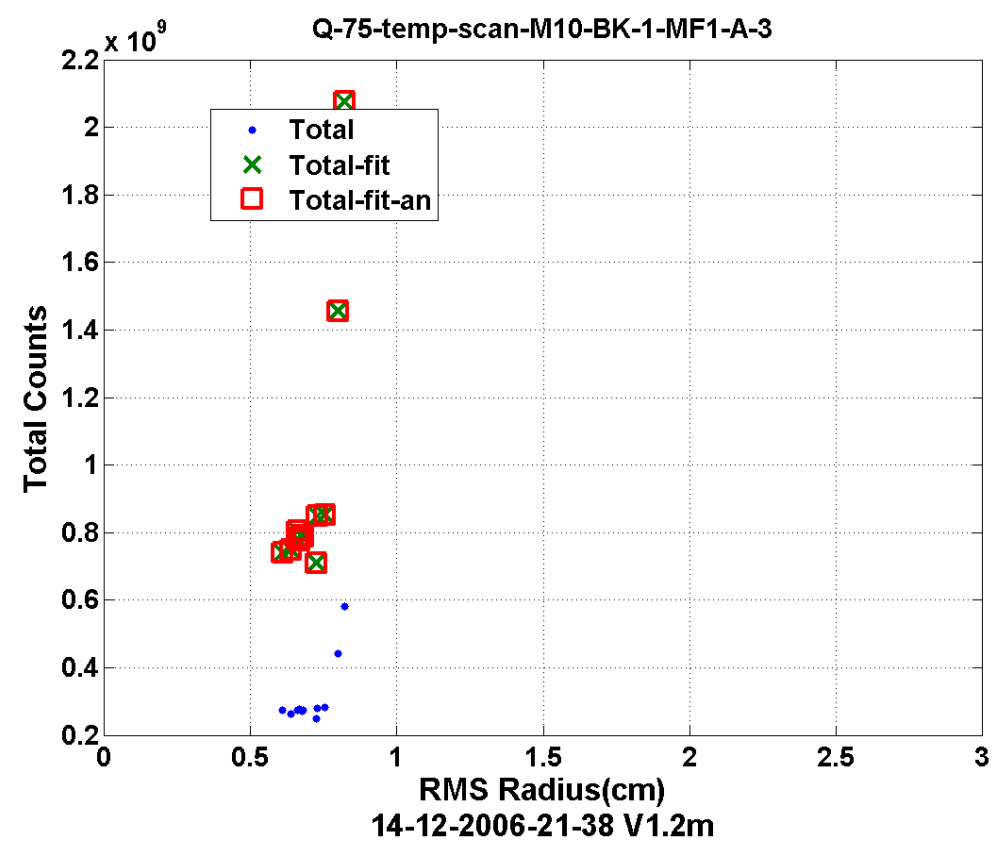

Figure 3.9: Total counts vs. RMS Radius for the Q75R1 repeat experiments. 'Total' indicates total counts from the foil after background subtraction, and 'Total-fit' indicates total counts calculated form the 1-D Gaussian fit of the data. ('Total-fit-an' is an internal check for 'Total-fit'). Both set of total counts increased with RMS radius but only slightly, after discarding the two data points at $r \sim 0.8-0.82$ due to saturation.

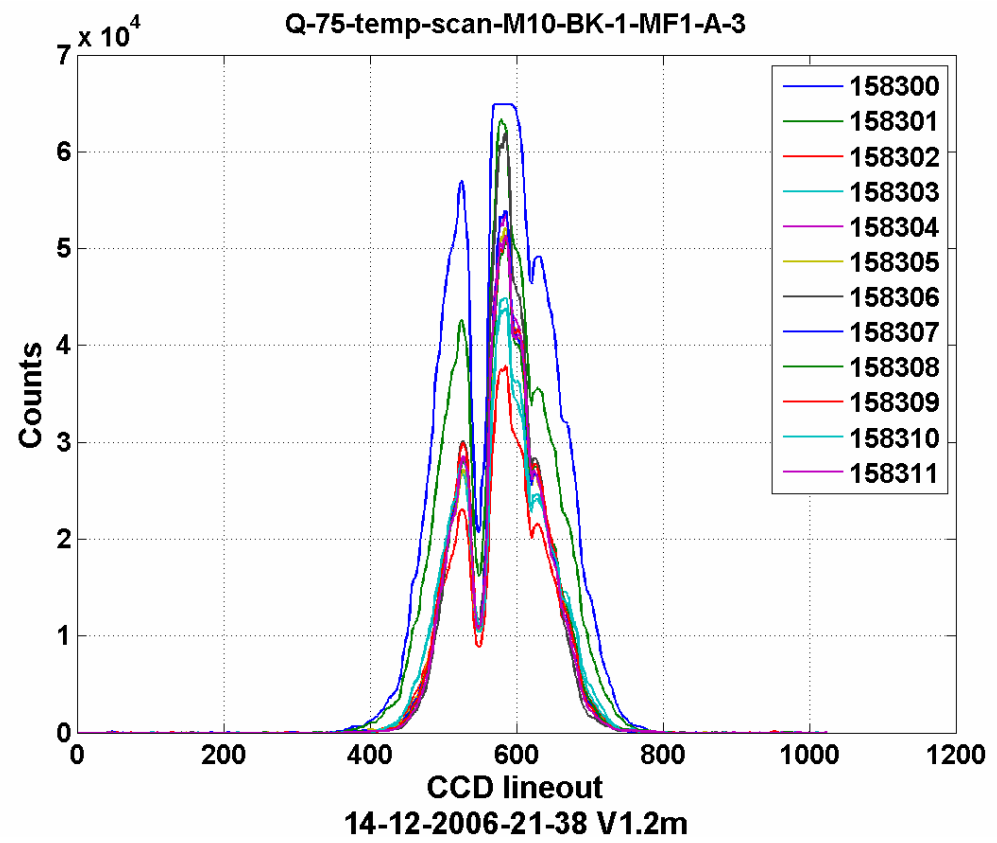

Figure 3.10: Summary of intensity data used to determine the RMS radius for the entire Q75R1 series. There is a general increased in peak intensity resulting in smaller RMS radii until the last shot. The first two shots had larger camera gains than the rest. 
Section IIIb: Kapton foil experiments on Dec. $15^{\text {th }}, 2006$.

On this day, four series of repeat experiments were done with a Kapton foil roughened on one side and smooth on the other side at nominally 45 degrees and 75 degrees. Here, the three 45 degree scans are examined. The settings used was a Rex06 tune with DR1\&2 at $350 \mathrm{~A}$ and L43\&44 at $300 \mathrm{~A}$. The first set of experiments, spanning 158393-158407 and dubbed K45R1, had the smooth side of the Kapton foil at 55 degrees to the beam with an f-stop of 5.60 and resulted in a hollow OTR profile which was not observed before. Currently, it is not clear why this hollow profile occurred. Figures 3.11 and 3.12 show some of the images for those shots and also their total counts. There was a $\sim 20$ minute delay between the fourth and fifth shot as the injector was switched from $56 \mathrm{kV}$ to $54 \mathrm{kV}$; otherwise the shots were a minute apart. As discussed, those shots always resulted in noticeably more total counts for currently unknown reasons.

The second repeat series performed, spanning shots 158408-158418 and dubbed K45R2, involved the Kapton foil at nominal 45 degrees with the rough side to the beam. Each shot was a minute apart. The same machine settings were used but the hollow OTR signal was gone. Unfortunately, near the end of this repeat series, some damage to the foil occurred around one of the fiducial holes which made interpretation of these data and the data from the rest of the day difficult. In addition, the signal level was very low; a strong median filter setting was required for the automated toolset. The filter smears out the fiducial hole in the center of the foil but those points were manual excluded from the fit. Also, the centroid was manually chosen for the series. Figures 3.13-3.16 gives the analyzed data set. The data mostly repeated given the large experimental errors involved; distinct trends were not obvious as in the case of the quartz experiments. At best it appears some isolation of shot-to-shot temperature effects was achieved since the radius did initially increase with shot number while the total counts decreased with shot number; however, the radius and count trends reverse after shot four. Lastly, examination of the x$\mathrm{y}$ displacement data from probes upstream of the diagnostic cross show moderately consistent profiles between shots from K45R1 and K45R2. These data are plotted in Figures 3.17-3.18. 
The third repeat series performed with a Kapton foil at nominal 45 degrees spanned shots 158458-158465 a minute apart and dubbed K45R3. This was essentially a repeat of K45R2, except the F-stop used was 2.80 in stead of 5.60. This series occurred later in the day by which point the foil was significantly more damaged and thus the data is even more questionable. In general the RMS radii repeated with shot number and were comparable to K45R2, if slightly smaller. This series is summarized in Figure 3.19.

Lastly, Figure 3.20 shows the damaged Kapton foil after the run day.

In summary, some interesting results were seen with the Kapton tests. The radius data roughly repeated after the first shot series and no clear discernable trends are seen in contrast to the quartz data. This would support the conclusion that the Kapton foils do cool themselves relatively quickly in-between shots and hence slight if any shot-to-shot based temperature effects were isolated as determined from Table 3.1. As is typical however, the first shot in a series or the shot after a long break gave the most total counts. The total counts versus radius did not show a discernable trend since the radii were approximately the same. An anomalous hollow ring structure was seen in the repeat series where the beam impacted the smooth side of the Kapton foil. Lastly, analysis of the displacement monitor data showed moderately consistent profiles between shots in K45R1 and K45R2. 


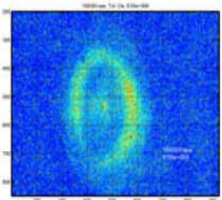

158393.png

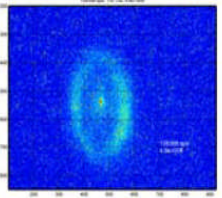

158398.png

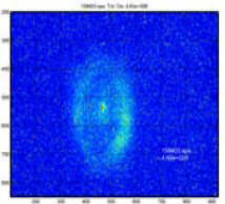

158403.png

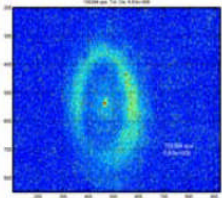

158394.png

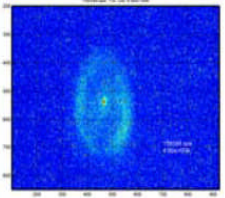

158399.png

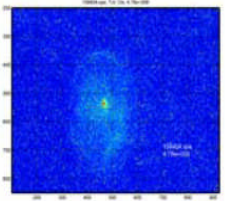

158404.png

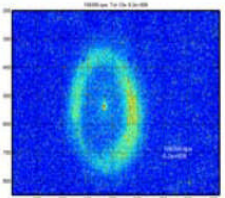

158395.png

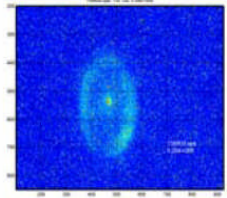

158400.png

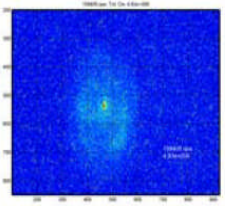

158405.png

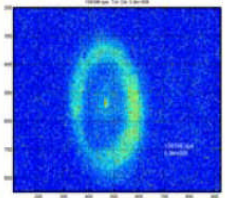

158396.png

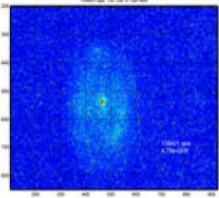

158401.png

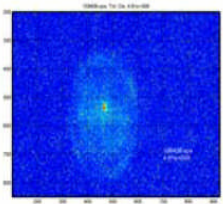

158406.png

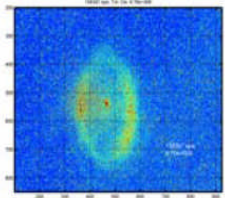

158397.png

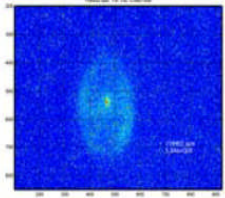

158402.png

Figure 3.11: Raw beam images from series K45R1, with smooth Kapton to the beam. Visually, the diameter of the anomalous hollow ring appears constant. The spot in the center stems from a fiducial hole.

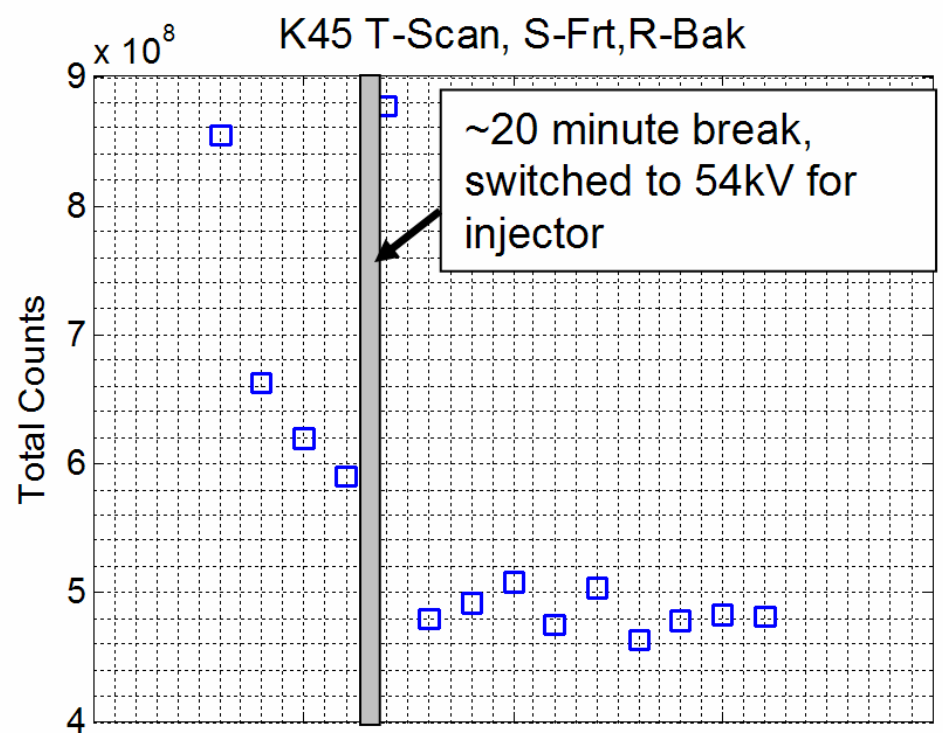

Figure 3.12: Total counts as a function of shot increment for series K45R1. Note the high total counts on the first and fifth shots. 

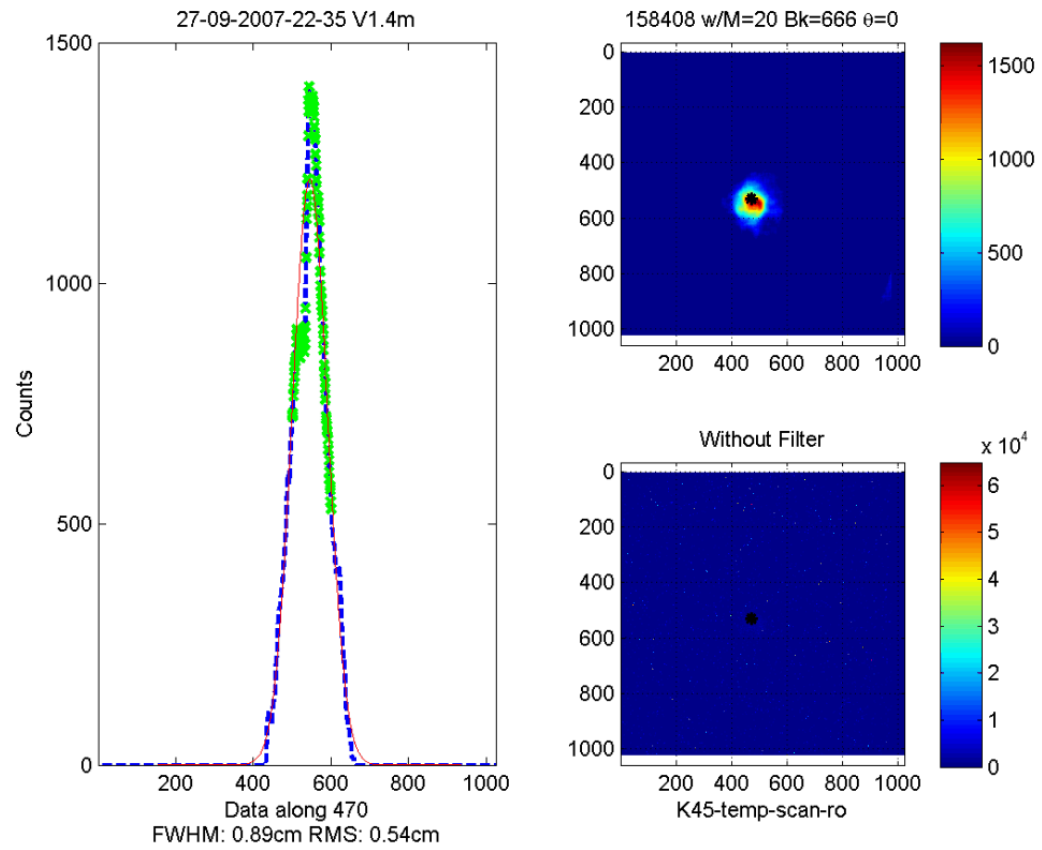

Figure 3.13: The first shot in the K45R2 repeat experiment. The green points were excluded from the Gaussian fit due to the fiducial hole and damage to that area that occurred later in this series. The fit gave a RMS radius of $0.54 \mathrm{~cm}$.

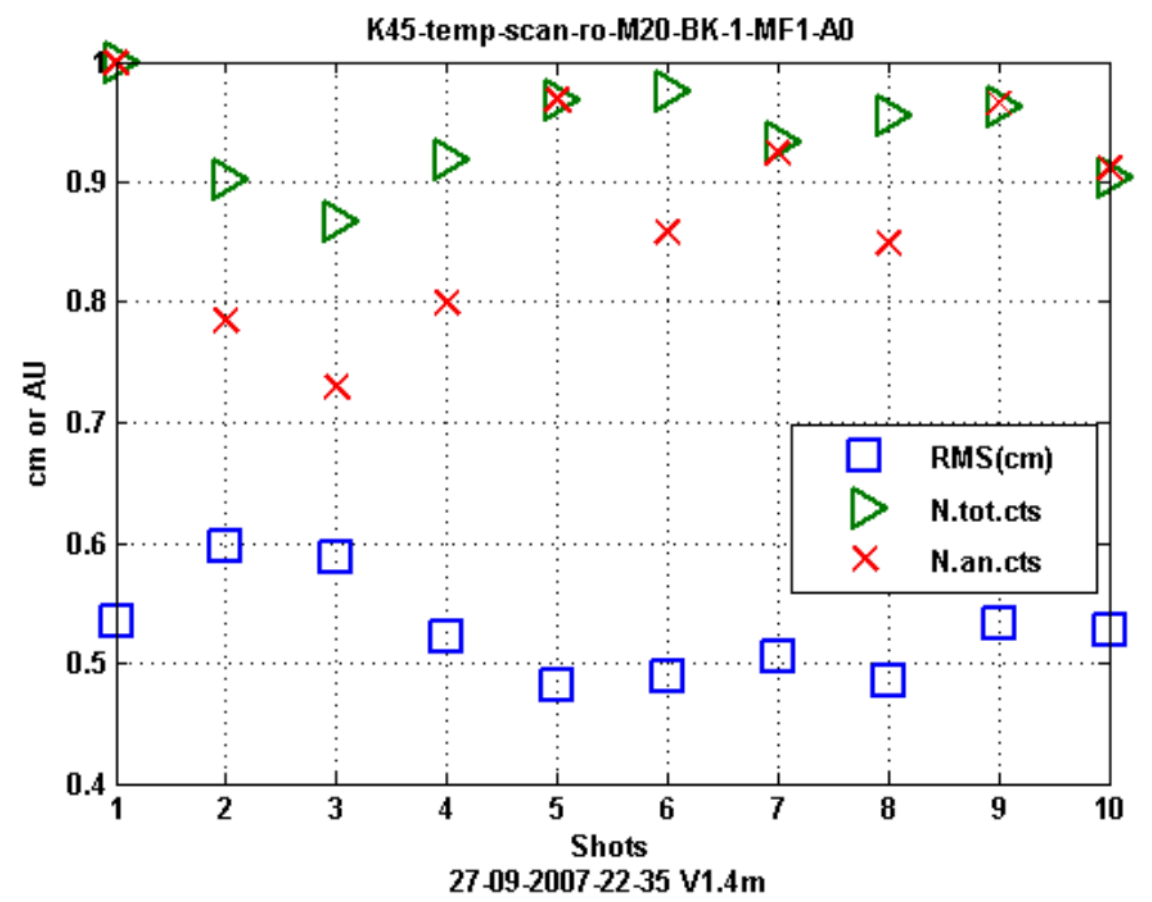

Figure 3.14: Summary of some key parameters in the K45R2 repeat experiments. The RMS radius data does not show a clear trend as in Figures 3.2 and 3.8. The other data shown are the normalized total counts from the foil and the normalized calculated total counts based on the analytical Gaussian fit. The count data is normalized to the first shot, with again the highest number total counts, but only slightly. 
LLNL-TR-402621

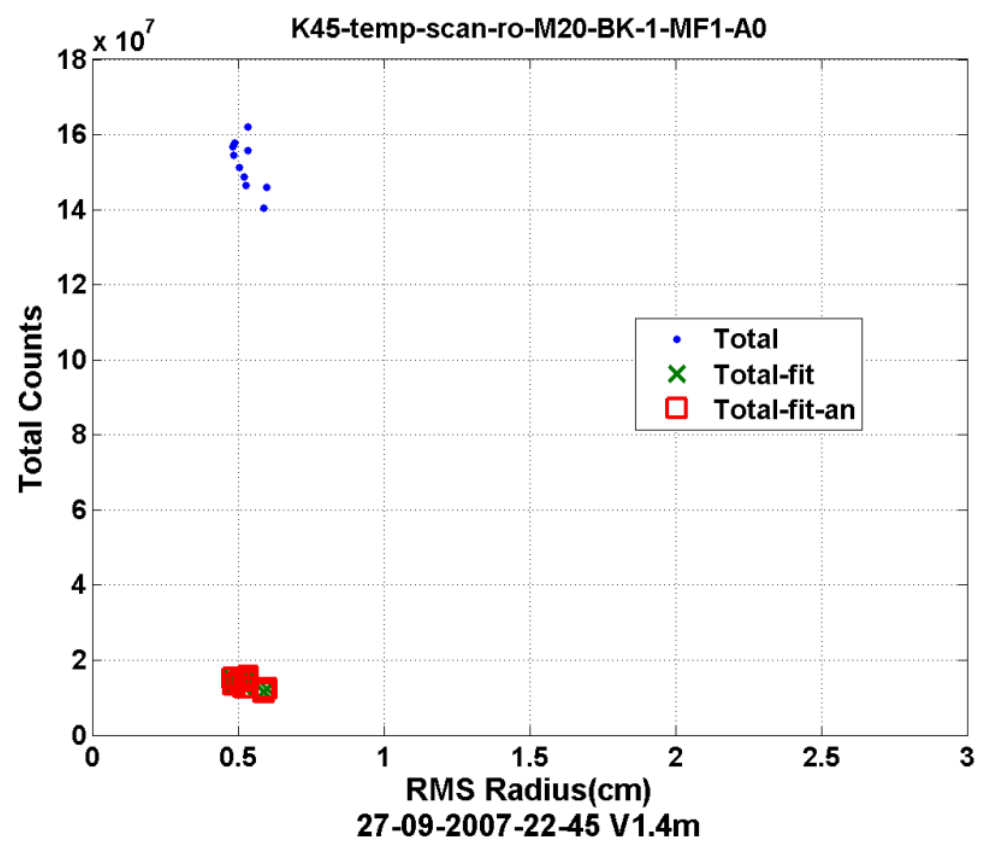

Figure 3.15: Total counts vs. RMS Radius for the K45R2 repeat experiments. 'Total' indicates total counts from the foil after background subtraction, and 'Total-fit' indicates total counts calculated form the Gaussian fit of the data. ('Total-fit-an' is an internal check for 'Total-fit'). There is no discernable trend in the data.

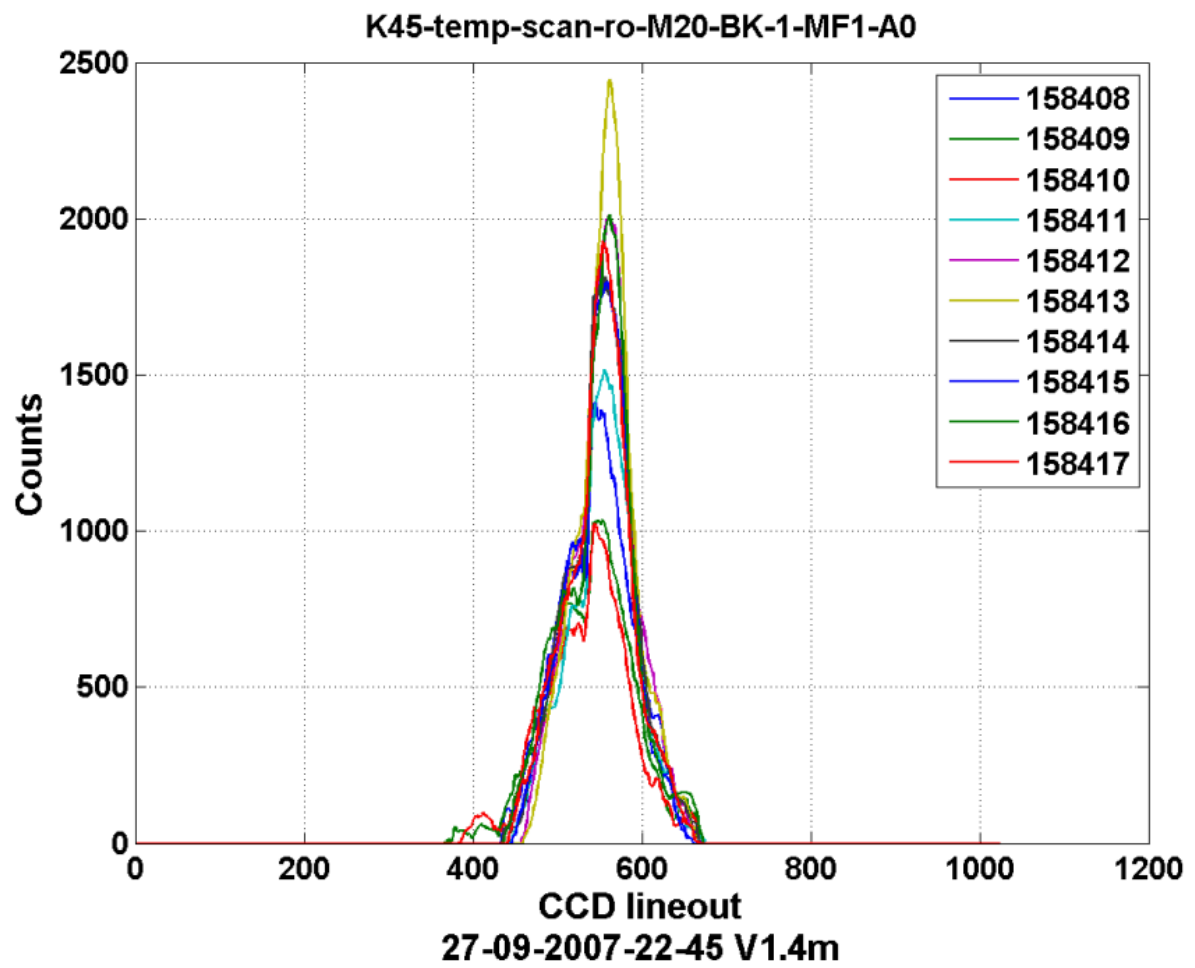

Figure 3.16: Summary of the intensity data used to determine the RMS radius for the entire K45R2 series. 
LLNL-TR-402621

BB-DR2AX

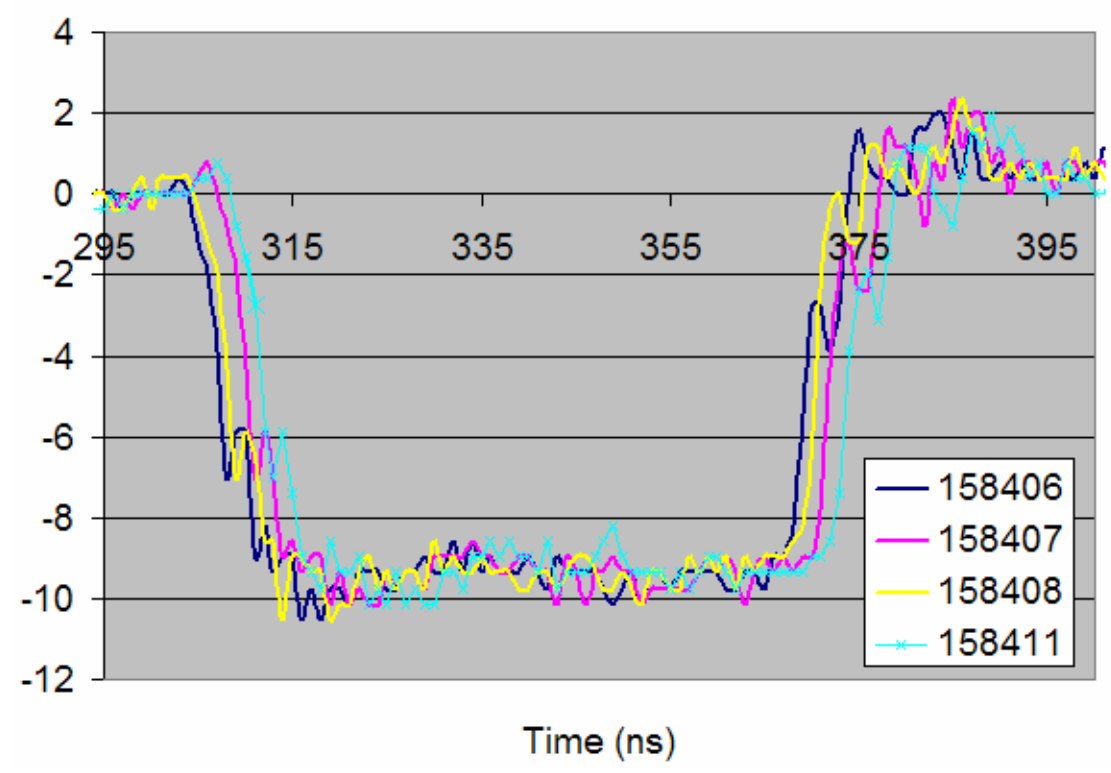

Figure 3.17: $\mathrm{x}$-displacement data from the DR2A probe upstream of the diagnostic cross for some of the shots in the K45R1 and K45R2 series. Shots 15406 and 158407 were the last shots in K45R1. 158408 was the first shot in K45R2, and 158411 the $4^{\text {th }}$ shot in $\mathrm{K} 45 \mathrm{R} 2$. The time base is relative and not absolute.

BB-DR2AY

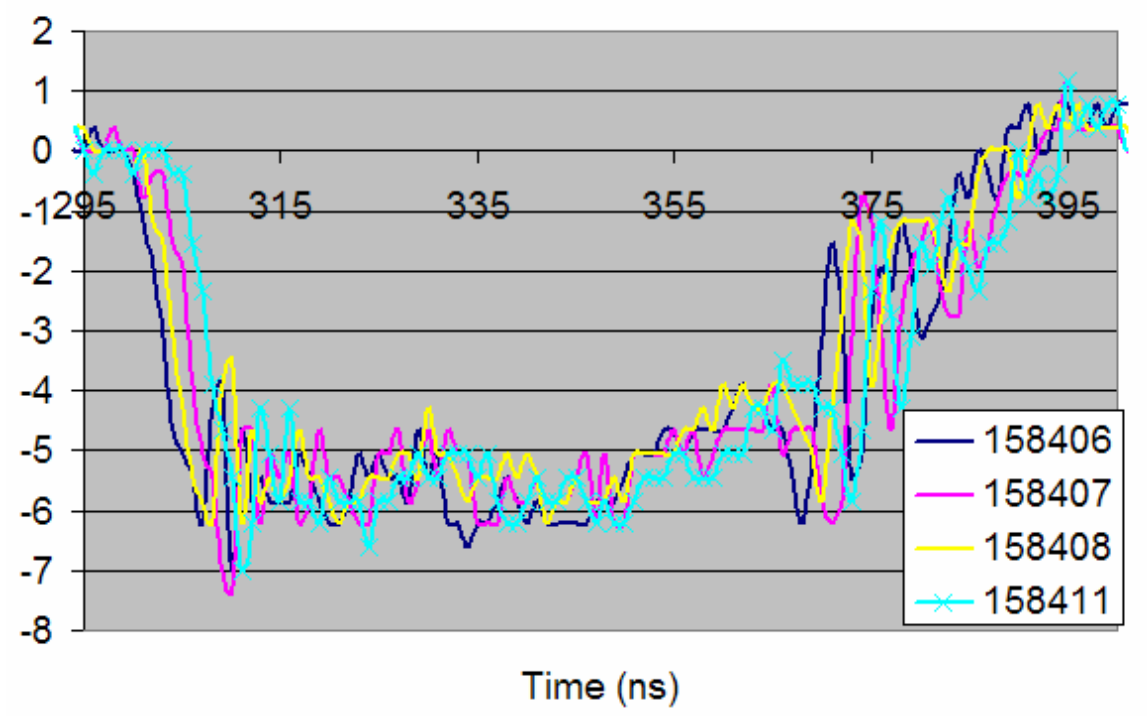

Figure 3.18: y-displacement data from the DR2A probe upstream of the diagnostic cross for some of the shots in the K45R1 and K45R2 series. Shots 15406 and 158407 were the last shots in K45R1. 158408 was the first shot in K45R2, and 158411 the $4^{\text {th }}$ shot in K45R2. The time base is relative and not absolute. 


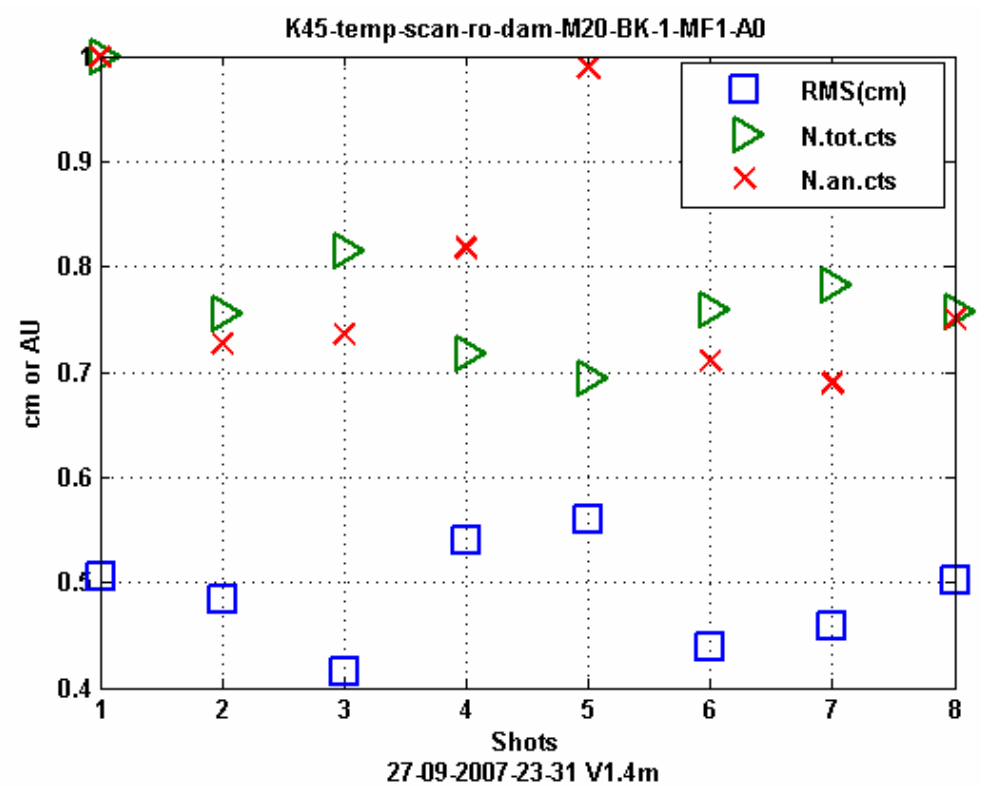

Figure 3.19: Summary of some key parameters in the K45R3 repeat experiments. This series was a repeat of K45R2 that occurred later on the same run day. The RMS radii are slightly lower than in the K45R2 experiment but the foil was also significantly more damaged at this point. The other data shown are the normalized total counts from the foil and the normalized calculated total counts based on the analytical Gaussian fit. The first shot is again used for the normalization and has the highest total counts.

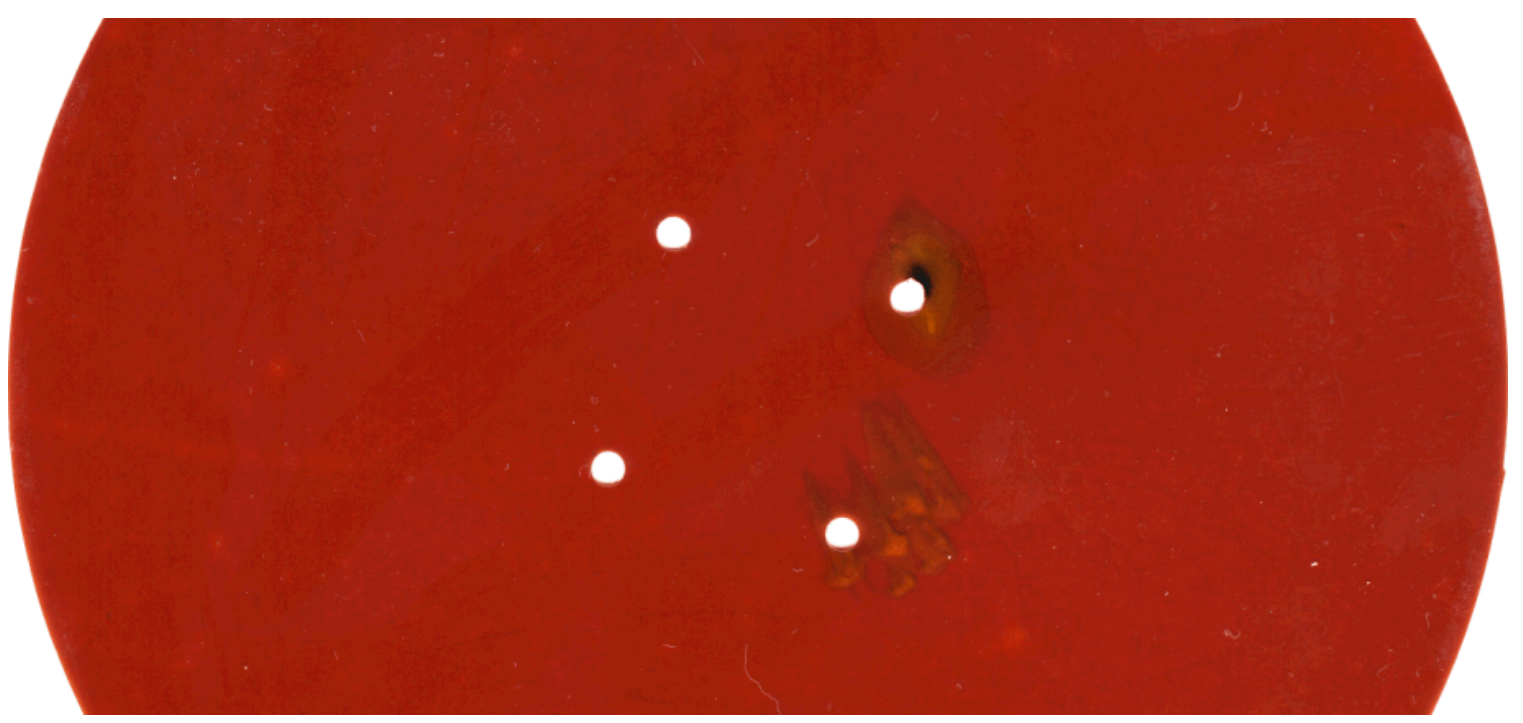

Figure 3.20: Damaged Kapton foil after Dec. $15^{\text {th }}, 2006$ run day. 
LLNL-TR-402621

Section IIIc: Al coated quartz foil experiments on Feb. $9^{\text {th }}, 2007$.

Benchmarking tests using a laser aligned 45 degree $\mathrm{Al}$ coated quartz foil were performed on this day. The objectives of these tests were to provide a set of benchmarking radius data for the dielectric foils repeat experiments. If the beam shots truly repeated and the foil was not perturbative, the radii for a set of repeated shots should be approximately the same, and no discernable trends should be seen.

Three repeat series were performed with the same magnet settings, namely a tune with DR1\&2 at $350 \mathrm{~A}$ and L43\&44 at $300 \mathrm{~A}$. The first series, AlR1 spanning shots 158979158992 and timed 2 minutes apart to avoid damage based on results using the model from Section II, was the first series of the day and shown discernable drifts in the radius and centroid data that suggests repeatability issues during the series. One good outcome is that the calculated total counts from the analytical fits matched the real total counts closely. The data are summarized in Figures 3.21-3.25. Temperature effects are doubtful because of the metallic foil and because a steady-state temperature should have been reached much quicker than the drift would indicate. It is likely that the machine needed a series of warm-up shots before repeatable shots can occur; this matches the results uncovered with the repeat runs for the dielectric foils and discussed previously.

The second set of repeat shots, spanning 159021-159039 and dubbed AlR2, occurred during the $5^{\text {th }}$ series of the day and tested the above theory that warm-up shots were necessary in order to produce a series of good repeats on FXR. Temperature based effects were isolated by first doing 10 shots with the foil withdrawn and then with the foil reinserted for the next 10 shots. This repeat series was timed 3 minutes apart in order to accomplish the task of manually inserting the foil in-between the tenth and eleventh shot. The data show the shots and OTR images to be much more repeatable, thus supporting the need for repeated warmed-up shots on FXR. Figures 3.26-3.28 give some of the key results. 
The last repeat series, spanning shots 159049-159058 and dubbed AlR3, followed and was a repeat of AlR1 except the 3 minute timing was maintained from AlR2. The data in this final series show good beam and OTR image reproducibility again and are summarized in Figures 3.29-3.30. Lastly, no foil damage occurred on this run day.

Hence, at a minimum, the data on this day show that a warmed-up machine timed 3 minutes apart can give shots that are very reproducible. The warmed-up period could be as short as $\sim 15$ repeat shots, based on the fact that the radius at the end of the AlR1 series in Figure 3.22 is closed to the steady radii seen in Figure 3.26. The difference between 2 to 3 minutes in the series should not be very critical in terms of uncovering any temperature related shot-to-shot variability in a series since the radiation model in Section II predicts that in both cases a steady-state post shot temperature should be reach within the first few shots, far faster than the slow radius droop given in Figure 3.22. In addition, the cooling period limits set by the radiation model from Section II were successful at preventing foil damage and allowed good data to be collected during this run day.
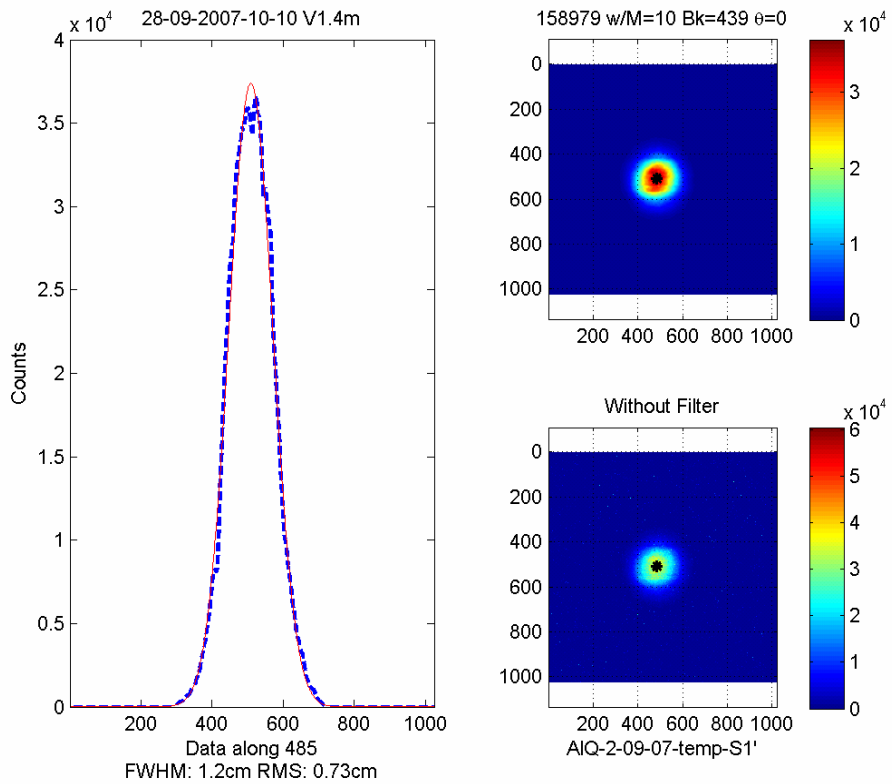

Figure 3.21: The first shot in the A1R1 repeat experiment. Copious signal levels were available and the very clean OTR data were taken. The Gaussian fit gave an RMS radius of $0.73 \mathrm{~cm}$. 
LLNL-TR-402621

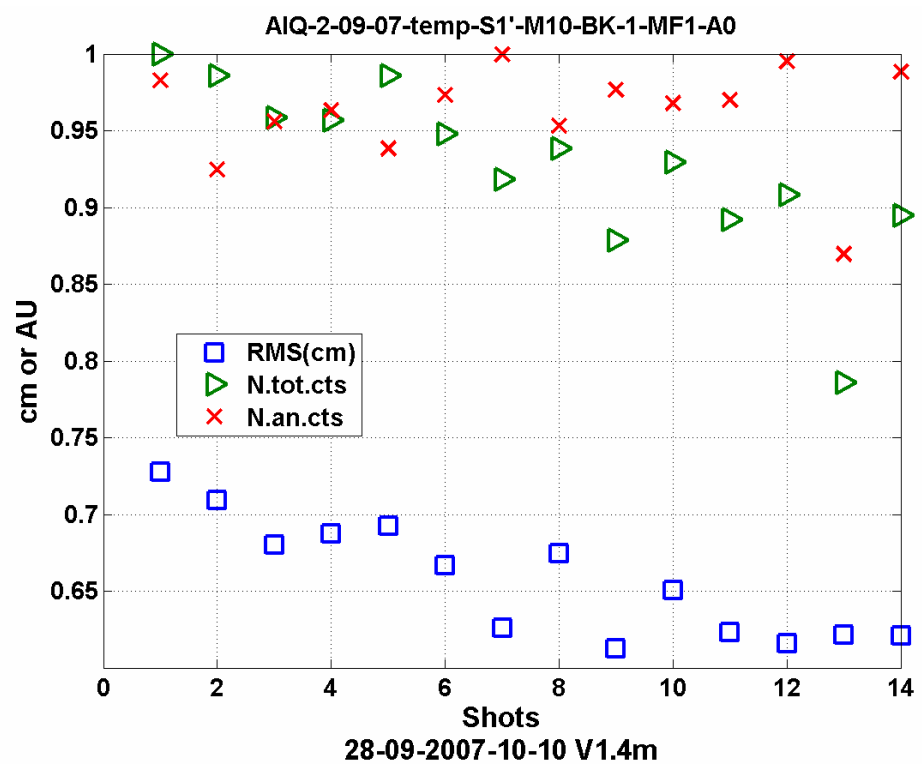

Figure 3.22: Summary of some key parameters in the AlR1 repeat experiments. The drift in the RMS radii correlate with drifts in the image centroid and could indicate repeatability issues on FXR when warm-up shots are not performed. The other data shown are the normalized total counts from the foil and the normalized calculated total counts based on the analytical Gaussian fit. The first shot again has the highest total counts. Interestingly the calculated normalized counts do not follow the decreasing trends in the real normalized counts, possibly indicating that the decrease in real total counts shot-to-shot might be due to reduced background sources such as x-rays for smaller beams.

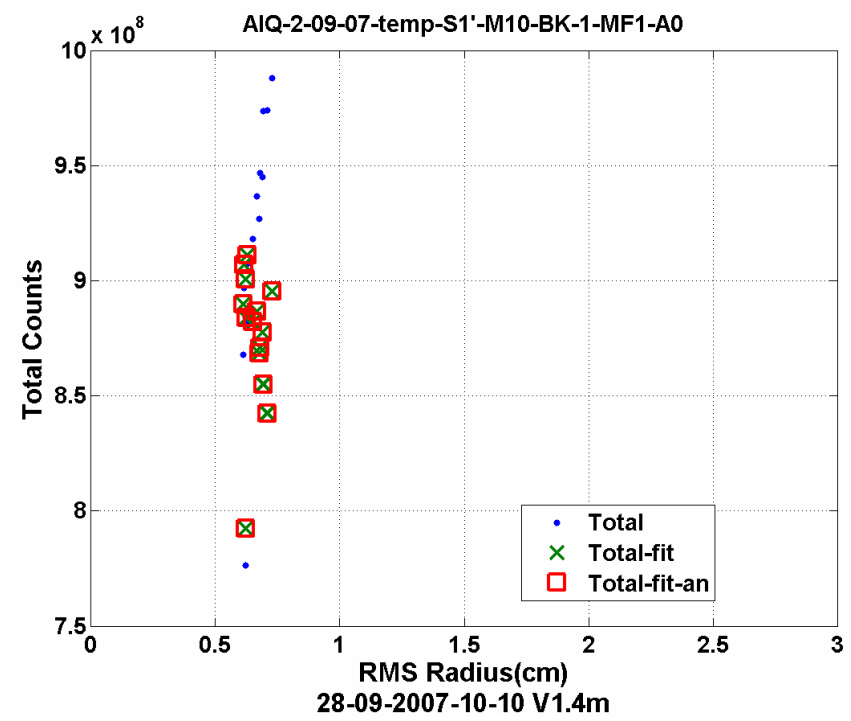

Figure 3.23: Total counts vs. RMS Radius for the AIR1 repeat experiments. 'Total' indicates total counts from the foil after background subtraction, and 'Total-fit' indicates total counts calculated from the Gaussian fit of the data. ('Total-fit-an' is an internal check for 'Total-fit'). There is some indication of increasing total counts versus increasing RMS radius in the Total data, but not in the Total-fit, thus possibly again suggesting decreased background noise such as x-rays from smaller beams. 
LLNL-TR-402621

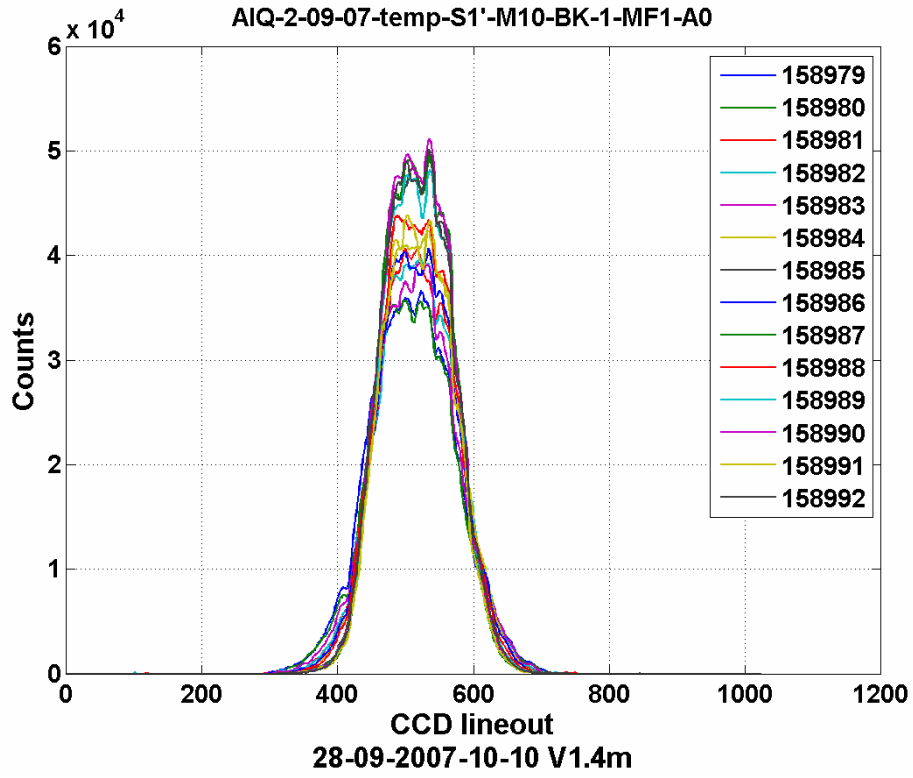

Figure 3.24: Summary of intensity data used to determine the RMS radius for the entire AlR1 series.

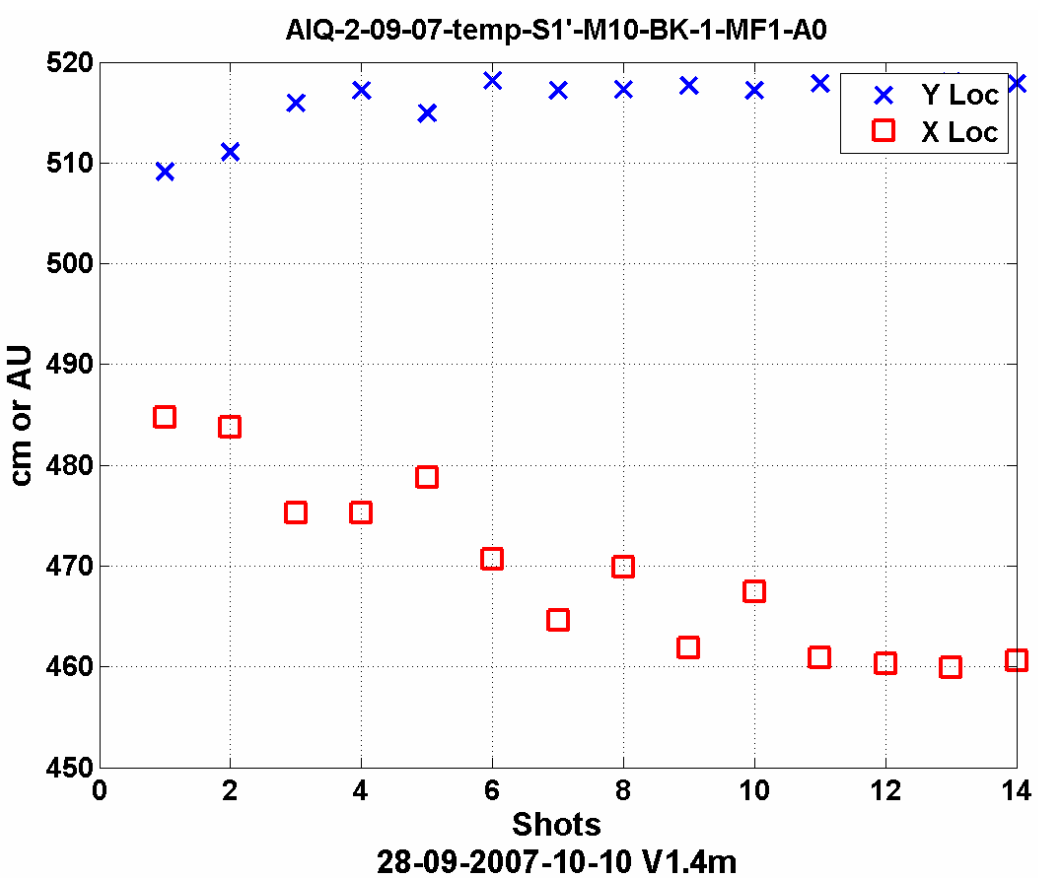

Figure 3.25: Summary of the image centroid for series AlR1. The drift in the centroid correspond to drift of the RMS radius data in Figure 3.22. 
LLNL-TR-402621

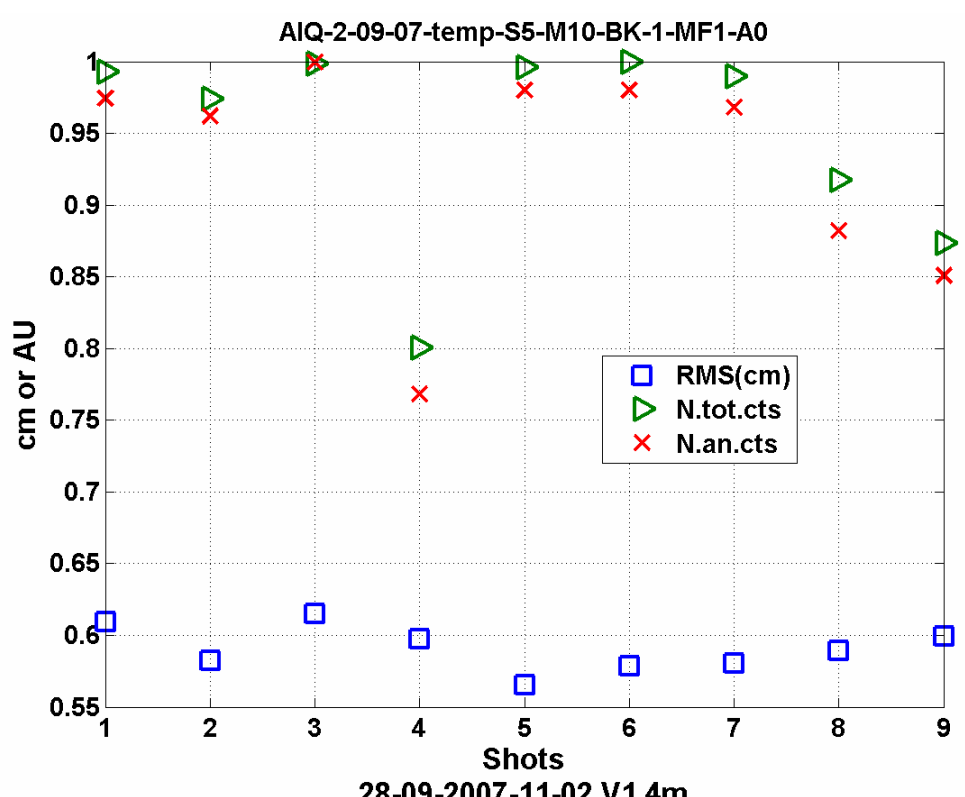

Figure 3.26: Summary of some key parameters in the AlR2 repeat experiments, where 10 warm-up shots occurred before the insertion of the Al coated quartz foil. The data shown are the RMS radius, normalized total counts from the foil, and the normalized calculated total counts based on the analytical Gaussian fit. The highest total counts in this series occurred in shot 3 . There are no discernable drifts in the radius or counts data, indicating good shot repeatability.

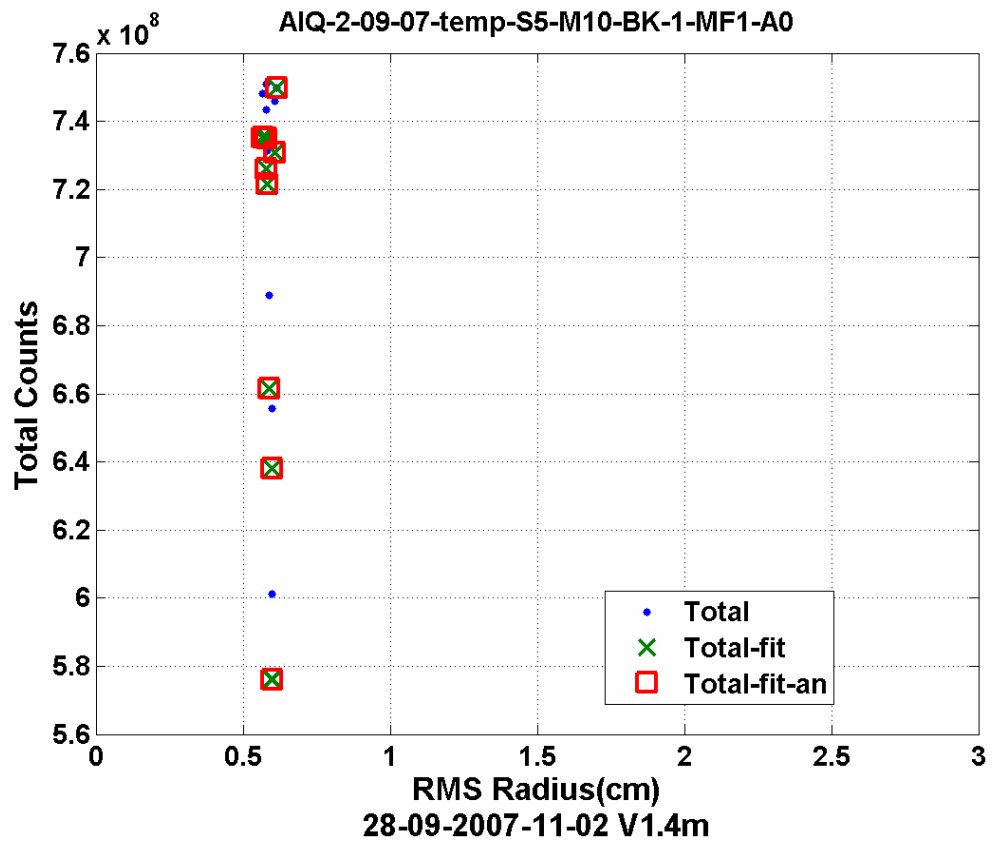

Figure 3.27: Total counts vs. RMS Radius for the AlR2 repeat experiments. 'Total' indicates total counts from the foil after background subtraction, and 'Total-fit' indicates total counts calculated from the Gaussian fit of the data. ('Total-fit-an' is an internal check for 'Total-fit'). Decent shot repeatability is again indicated by this data. 
LLNL-TR-402621

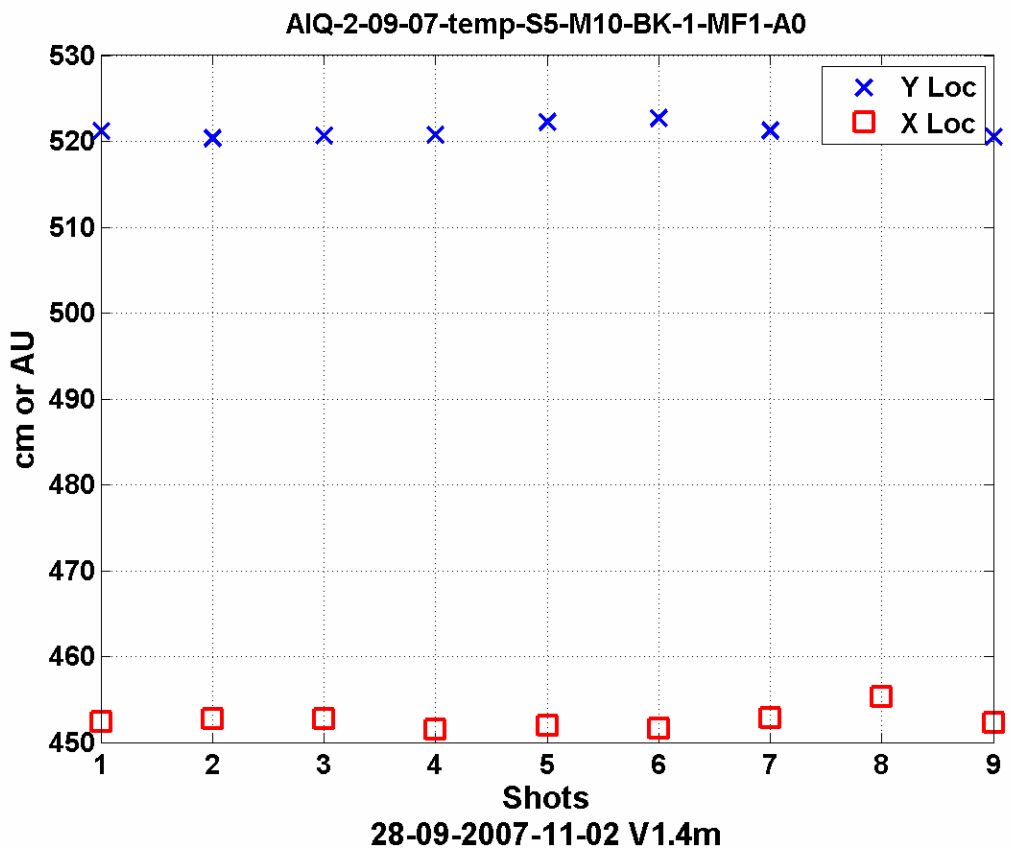

Figure 3.28: Summary of the image centroid for series AlR2. There is little drift in the data indicating good shot repeatability.

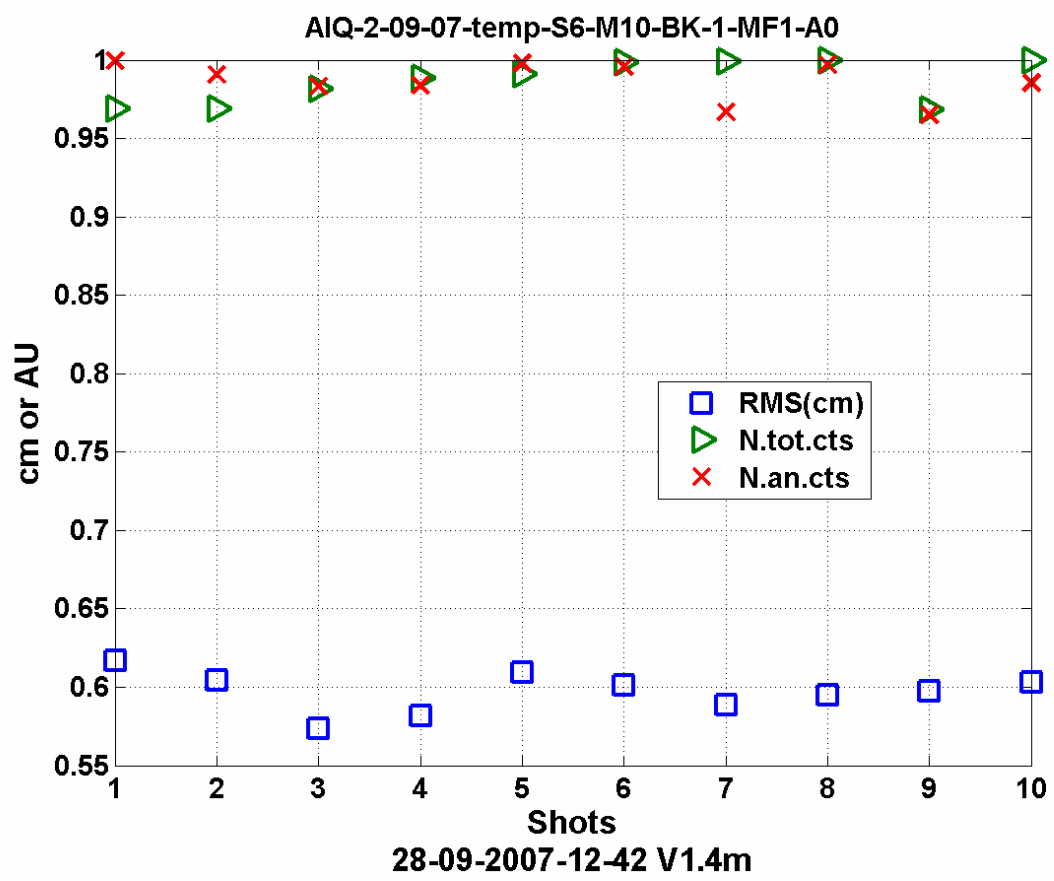

Figure 3.29: Summary of some key parameters in the AlR3 repeat experiments. The data shown are the RMS radius, normalized total counts from the foil, and the normalized calculated total counts based on the analytical Gaussian fit. There are no discernable drifts in the radius or counts data, indicating good shot repeatability. 
LLNL-TR-402621

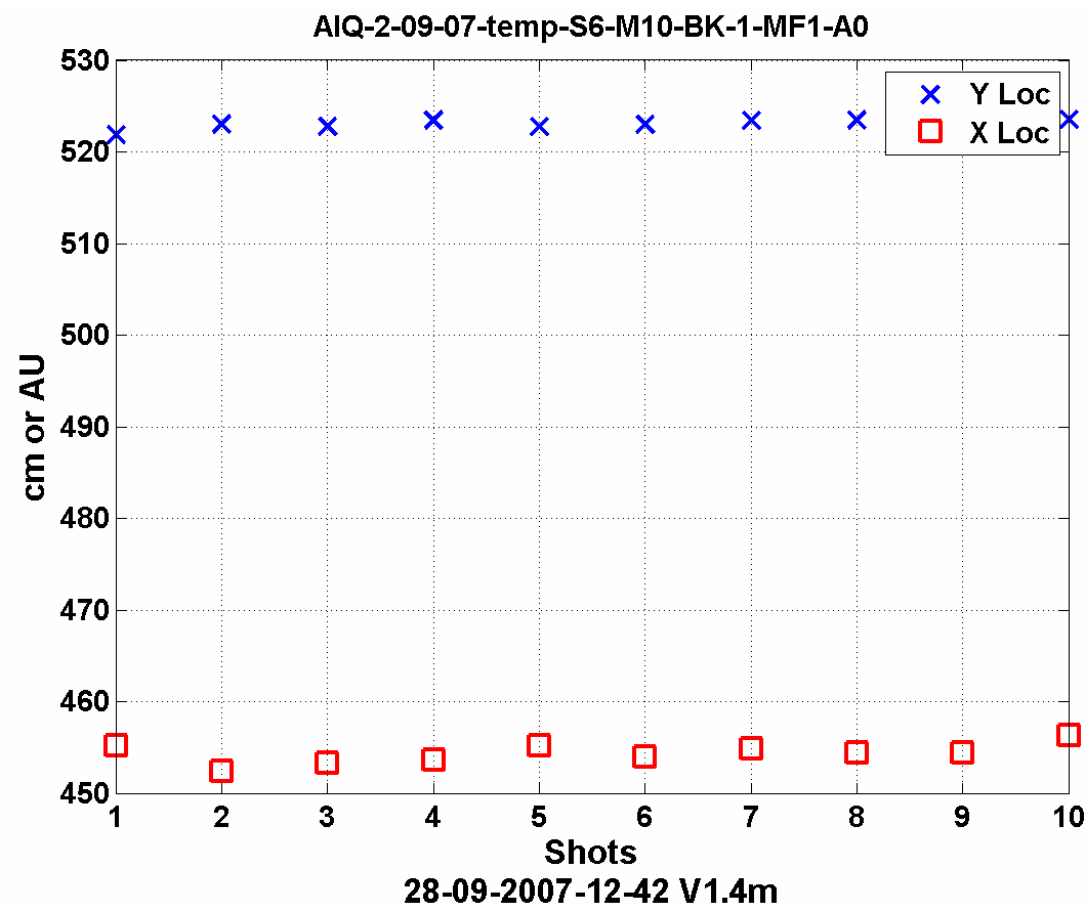

Figure 3.30: Summary of the image centroid for series AlR2. There is little drift in the data indicating good shot repeatability.

Section IIId: Al coated quartz foil experiments on Mar. $16^{\text {th }}, 2006$.

On this day, a limited set of repeat shot benchmarking data with a laser aligned 45 degree Al coated quartz foil were performed. Two series were performed, both as the first and second sets of the run day. A rex06 tune with DR1\&2=350 A and L43\&44=300 A was used.

The first series spanning shots 159260 to 159274 two minutes apart and dubbed AlR4 gave similar results to series AlR1 from Feb. $9^{\text {th }}, 2006$ in that the image centroid and RMS radius drifted throughout the series, again suggesting that warm-up shots are necessary for repeatability. Figures $3.31-3.35$ give a summary.

Slight defects in the camera image in the first series caused by some lint fiber on the diagnostic lens prompted an attempt by Tang and Watson to clean the lens with clean compressed air in between the first and second shot series. Unfortunately, this made the 
situation worse by clouding the lens. A break of 15 minutes in between the first and second series occurred as a result.

The data on the second series spanning shots 159275 to 159289 and dubbed AlR5 show good image reproducibly, but the radii were consistently larger than expected and larger than the first series. This was probably caused by the cloudiness in the lens. Figures 3.36-3.37 provides the key parameters.

Overall, the data on this day provides additional evidence for shot repeatability issues when warm-up shots are not performed.
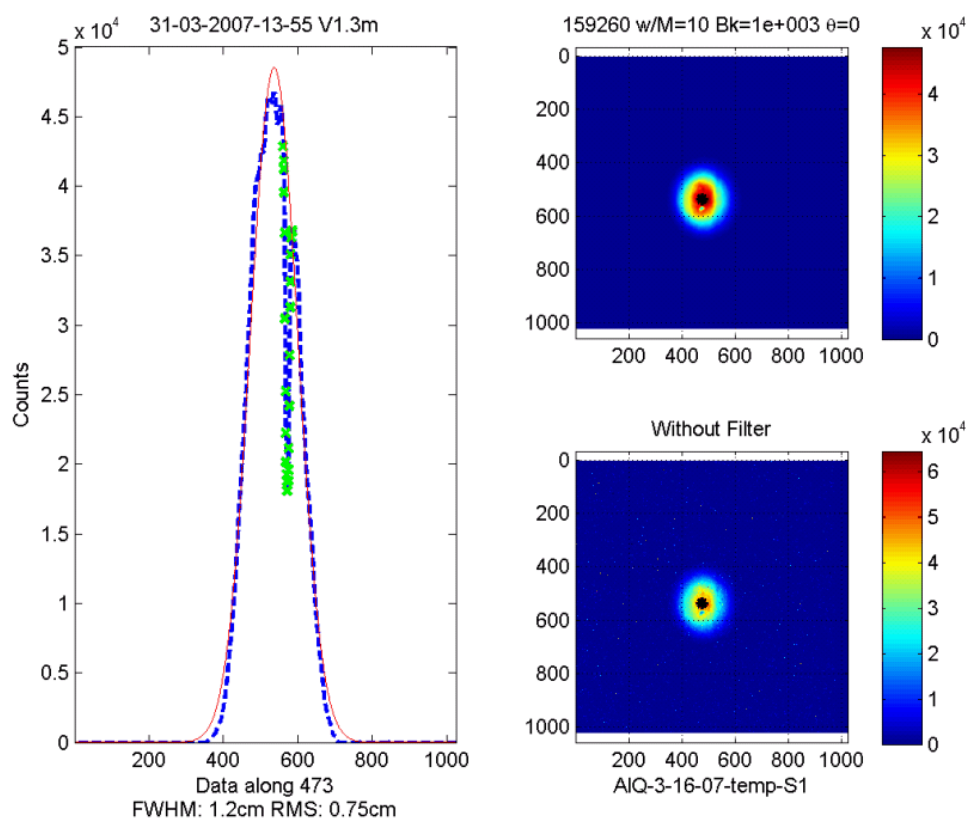

Figure 3.31: The first shot in the A11R4 repeat experiment. Copious signal levels were available and the very clean OTR data were taken. The Gaussian fit gave an RMS radius of $0.75 \mathrm{~cm}$. 
LLNL-TR-402621

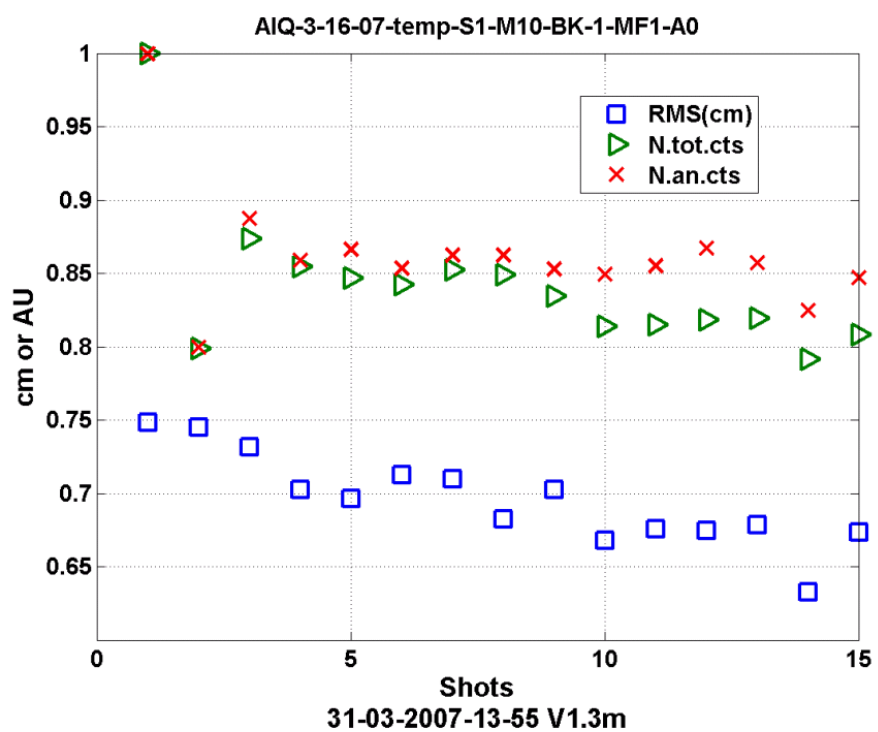

Figure 3.32: Summary of some key parameters in the AlR4 repeat experiments. The drift in the RMS radii correlate with drifts in the image centroid given in Figure 3.34 and could indicate repeatability issues on FXR when warm-up shots are not performed. The other data shown are the normalized total counts from the foil and the normalized calculated total counts based on the analytical Gaussian fit. The first shot again has the highest total counts. Interestingly the calculated normalized counts do not exactly follow the decreasing trends in the real normalized counts, possibly indicating that the decrease in real total counts shot-to-shot might be due to reduced background sources such as $\mathrm{x}$ rays for smaller beams.

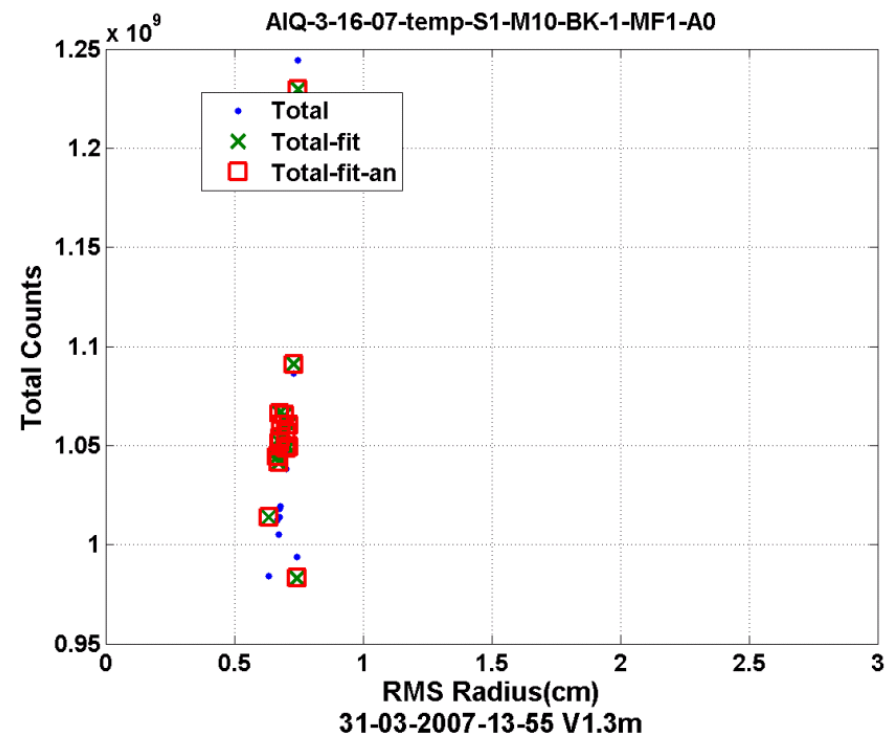

Figure 3.33: Total counts vs. RMS Radius for the AlR4 repeat experiments. 'Total' indicates total counts from the foil after background subtraction, and 'Total-fit' indicates total counts calculated from the Gaussian fit of the data. ('Total-fit-an' is an internal check for 'Total-fit'). There is some indication of increasing total counts versus increasing RMS radius in the Total data, but not in the Total-fit, thus possibly again suggesting decreased background noise such as X-rays from smaller beams. 
LLNL-TR-402621

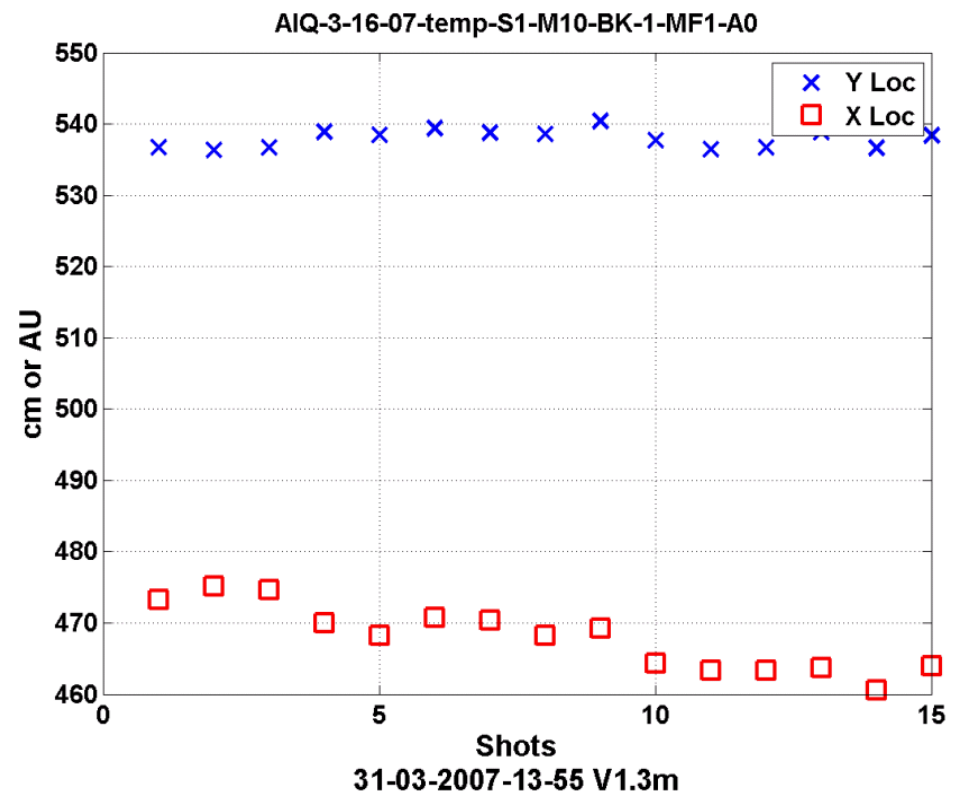

Figure 3.34: Summary of the image centroid for series AlR4. The drift in the centroid correspond to drift of the RMS radius data in Figure 3.32.

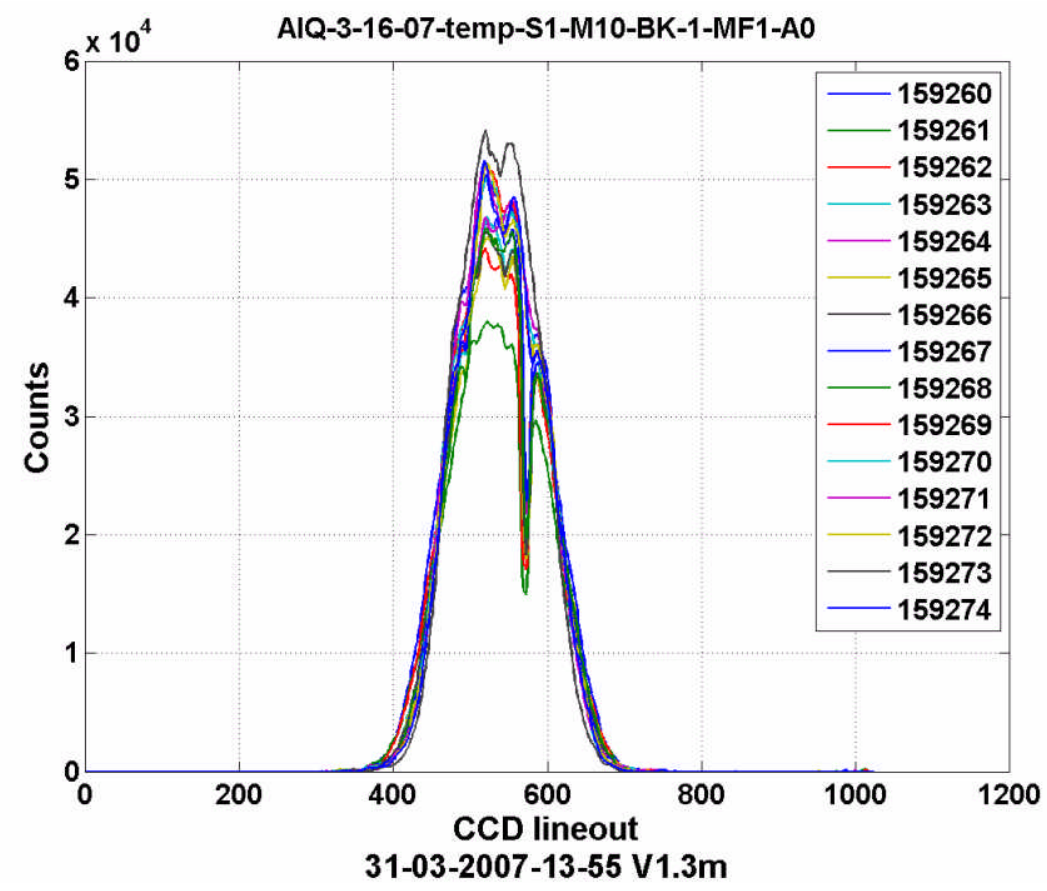

Figure 3.35: Summary of intensity data used to determine the RMS radius for the entire AlR4 series. 


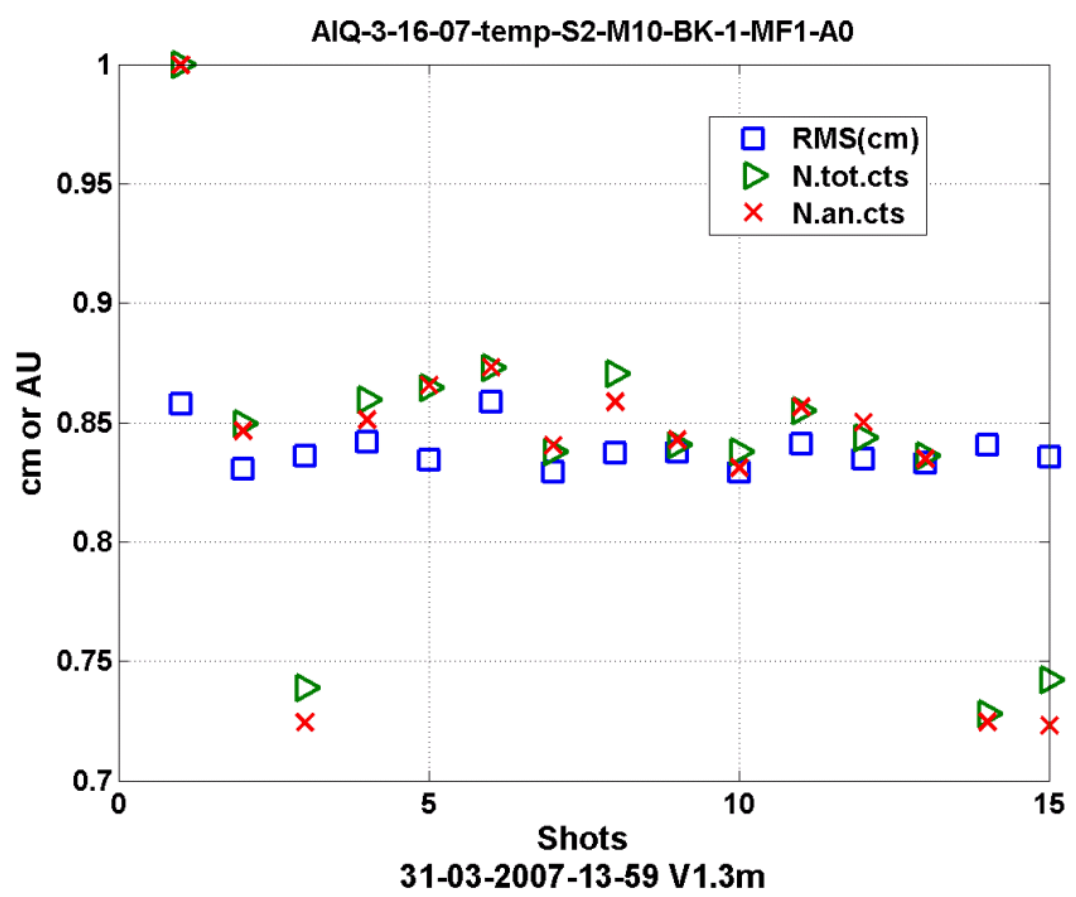

Figure 3.36: Summary of some key parameters in the AlR5 repeat experiments. The data shown are the RMS radius, normalized total counts from the foil, and the normalized calculated total counts based on the analytical Gaussian fit. The image radii are relatively large and the data should be only interpreted qualitatively due to cloudiness in the lens.

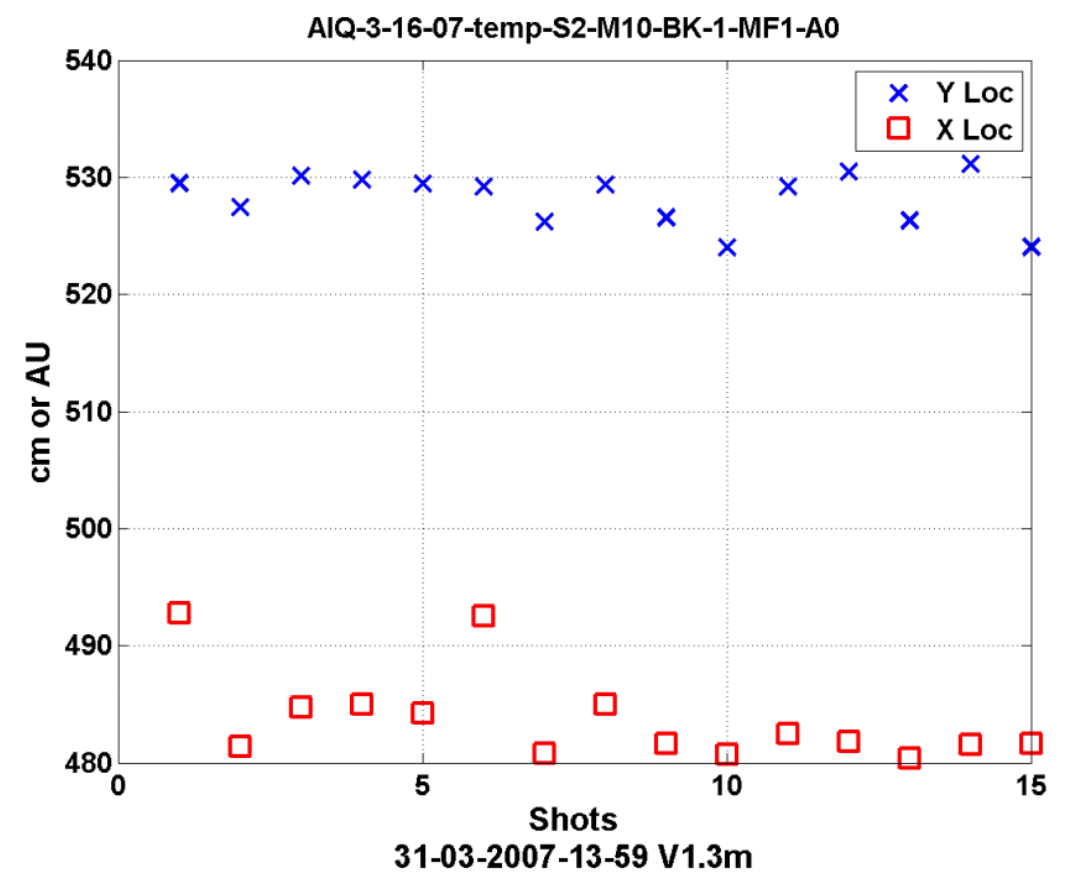

Figure 3.37 Summary of the image centroid for series AlR5. The data in this series was affected by a cloudy lens. 


\section{Section IV: Synthetic OTR Diagnostic}

A synthetic OTR diagnostic simulation for smooth dielectric and metallic foil was created which identified and resolved some OTR spot interpretation issues. The ultimate goal of this tool is to provide the interpretative modeling necessary to determine accurate beam profile data from spot-size OTR data, with verification of the simulations involving dielectric foils coming from the benchmarking experiments such as the ones discussed in Section III. The tool is preliminarily operational and some results are available.

As discussed in the introduction of this report, currently only smooth dielectric foils can be simulated as modeling rough foils would require an accurate scattering model to determine the amount of backscattered Cerenkov radiation detected and the degree of anisotropy that would be eliminated for both OTR and Cerenkov radiation. Also, the simulations currently model only ideal (cold) non-divergent beams, or low emittance beams at a waist; divergence and emittance effects can be accounted for and are future work. Depending on how divergent and hot the beam is, these effects can be important. For the metallic foils or foils with metallic coatings considered here, the Cerenkov radiation is not an issue since light emitted from within the foil can not escape. Overall, the simulations at this stage have already proven useful as they have shed light on the intricacies of the OTR diagnostic.

Concerning those insights, a geometric factor was found in the diagnostic response function which varies with radius and plays a role in interpreting both smooth dielectric and metallic foil spot-size OTR. This is in addition to temperature based effects. The geometric effect affects the radius interpretation of the spot data more than the temperature based effects. However, the temperature effects are clearly seen in simulations of the total counts. Spot RMS radius versus real beam RMS radius plots for ideal non-divergent beams are now available for both metallic and Kapton foils. So far, fortuitously, the spot RMS radii and the beam RMS radii in the simulations are not too far apart when an optimum optical setup with low f number is used. In other cases, such as an optical setup with high f-number, the synthetic diagnostic results show that the 
observed spot-size and radii can be noticeably larger than the real beam radii, due to both the anisotropic nature of OTR and the studied temperature effects. Lastly, a qualitative match was found between an experimental and simulated total counts versus spot RMS radius curve for some of the Kapton data. For the cases examined, the emission intensity versus permittivity scaled with a more complicated quadratic than the previously assumed $|\varepsilon-1|^{2}$.

A set of challenges identified from this beginning work on the dielectric modeling portions of the code is again the need for a foil which is either completely roughened or smooth for accurate simulations and the lack of accurate permittivity data as a function of temperature for the dielectric foils of interest.

This Section documents the synthetic diagnostic and the work done so far; it is an extension of reference [1]. 


\section{Section IVa: Model Details}

In this sub-section, the FXR OTR diagnostic setup and the details of the synthetic diagnostic are reviewed. A specific case of an ideal FXR beam with a non-divergent Gaussian current profile hitting a smooth $0.013 \mathrm{~cm}$ thick Kapton foil placed 45 degrees to the beam is first examined. In other words the beam is modeled as a stream of electrons at $17.6 \mathrm{MeV}$ with the same velocity vector and a Gaussian current density profile with user specified RMS radius.

The simulation consists of three parts; the first is made up of an OTR emission calculator for dielectric foils given certain angles, material data, and desired frequency range. The second consists of the model used to simulate the temperature dependent permittivity. The code currently uses a simple Debye permittivity model to account for temperature dependent changes in the dielectric foil. Only instantaneous heating is considered currently; the code can be easily modified to accept shot-to-shot based temperatures if required. The third portion couples the first two parts to simulate the detector or camera response and requires inputs such as current density profile for calculating the temperature profile and camera efficiency as a function of frequency.

\section{Diagnostic Setup and OTR emission}

The portion of the FXR OTR diagnostic considered here again primarily consists of a 60 $\mathrm{mm} \mathrm{f} / 2.8 \mathrm{D}$ Nikon collection lens $\sim 25 \mathrm{~cm}$ away from a rotatable $\sim 11 \mathrm{~cm}$ diameter OTR foil. Kapton or quartz dielectric foils can be employed. The signal is coupled via optical fibers to a fast CCD camera typically operated with gate widths of $\sim 2 \mathrm{~ns}$. Usually, the diagnostic is setup to view the specular reflection at 45 degrees. The setup is schematically illustrated in Figure 4.1. The synthetic diagnostic discussed here aims to simulate the detected OTR emission along a thin vertical strip at the center of a Kapton foil. 
LLNL-TR-402621
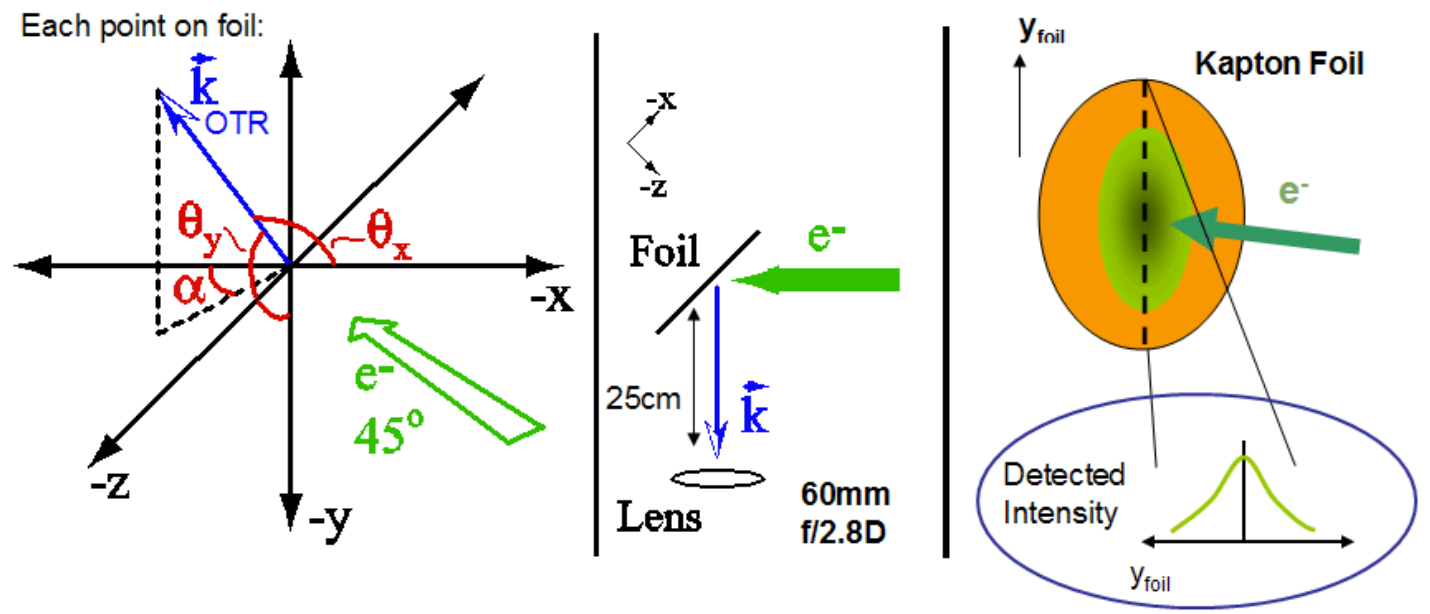

Figure 4.1: Coordinate and Experimental setup. The diagnosed beam traverses at 45 degrees a dielectric foil in the x-y plane. The OTR emission from each point on the foil (i.e. origin on the left) is represented by the vector $\mathrm{k}$ and varies depending on angles $\theta_{\mathrm{y}}$ and $\alpha$. A unit normal from the lens' center lies on the $\mathrm{x}-\mathrm{z}$ plane. Emission from the center of the foil detected using the lowest f-number of the lens (2.8) comes from a solid angle covering $\alpha=45 \pm \sim 2.5 \mathrm{deg}$ and $\theta_{\mathrm{y}}=90 \pm \sim 2.5 \mathrm{deg}$. The goal of the simulation is to predict the detected OTR curve qualitatively illustrated by the rightmost drawing.

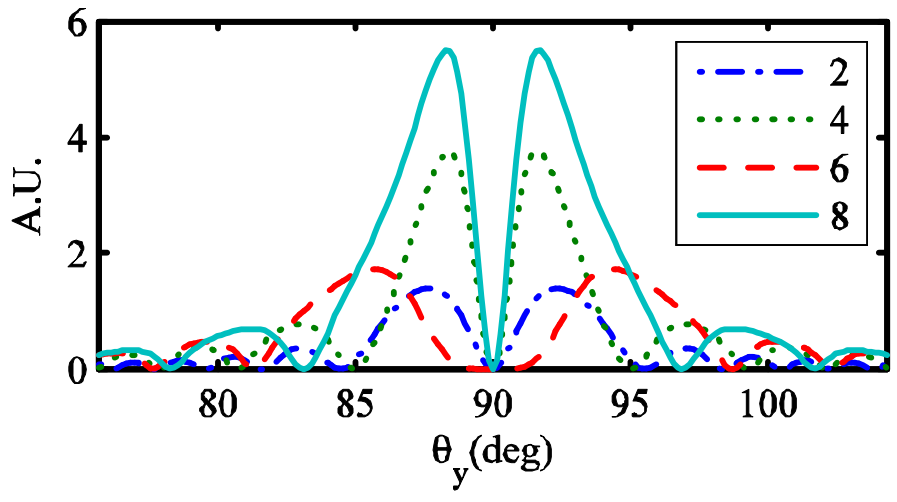

Figure 4.2: OTR emission at $2 \mathrm{eV}$ for a $0.1265 \mathrm{~mm}$ thick dielectric foil with $\varepsilon=2$ to 8 at $\alpha=45$ degrees.

The relevant OTR equations for dielectric foils with arbitrary geometry were noted by Ter-Mikaelian [2] and are cumbersome, in comparison with the OTR emission for a highly relativistic electron beam traversing a metallic foil, given by[3]:

$$
\frac{d^{2} I}{d \omega d \Omega} \sim \frac{e^{2}}{\pi^{2} c} \frac{\theta^{2}}{\left(\theta^{2}+\gamma^{-2}\right)^{2}}
$$

Where I is the emission intensity, $\theta$ is the angle with respect to the specular vector or $\theta_{\mathrm{y}}$ in Figure 4.1 when $\alpha$ is at the specular angle (i.e. 45 degrees), $\gamma \sim 34.3$ for FXR electron 
beams, $\omega$ the emitted light frequency and $\Omega$ the solid angle. The emission is peaked at $1 / \gamma$. In effect, for a non-metallic foil, complex interferometer effects occur since the front surface is no longer opaque to the back surface of the foil.

The permittivity dependence of the OTR emission, again assuming no beam divergence and temperature, can be seen in Figure 4.2. The emission is a sum of both perpendicular and parallel polarization. Integrating the emission over the relevant photon energies of 1.7 to $6.2 \mathrm{eV}$ with the FXR camera efficiencies averages out the interferometer effects in Figure 4.2 and results in the smooth profiles shown in Figure 4.3. The peak of the emission profiles is $\sim 1 / \gamma$, similar to OTR from metallic foils. The permittivity dependence is roughly proportional to $-\varepsilon^{2}+16 \varepsilon-15$ at the $1 / \gamma$ peak for $\varepsilon=1$ to 8 but varies moderately with $\theta_{\mathrm{y}}$ and $\alpha$.

The integrated dielectric OTR emission were benchmarked against analytical results for metallic foils and matched perfectly when the permittivity in the calculations was taken to infinity.

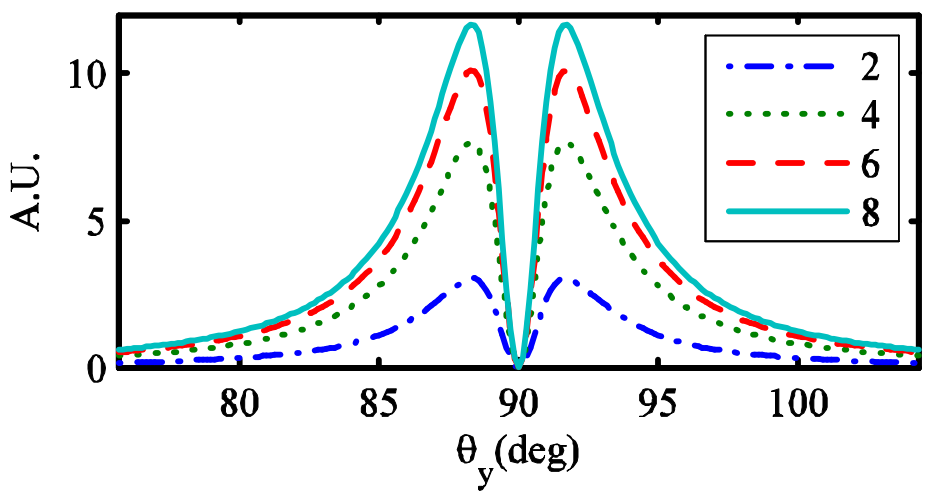

Figure 4.3: OTR emission from Figure 4.2 integrated from 1.7 to $6.2 \mathrm{eV}$ with camera efficiencies.

Temperature and Permittivity Profile

The temperature of the Kapton foil during a shot is modeled assuming again (from Section II) a constant heat capacity of $1.09 \mathrm{~J} / \mathrm{gm}-\mathrm{K}, \rho$ of $1.42 \mathrm{gm} / \mathrm{cc}$, and a foil heating of $\sim 3.4 \times 10^{13} \mathrm{~J} / \mathrm{cm}$ foil thickness per electron. For a Gaussian beam with $\mathrm{r}_{\mathrm{rms}}$ of $0.5 \mathrm{~cm}$ 
hitting a foil initially at room temperature, this results in a temperature profile mid-pulse shown in Figure 4.4. This profile can be used to determine temperature dependent permittivity OTR effects.

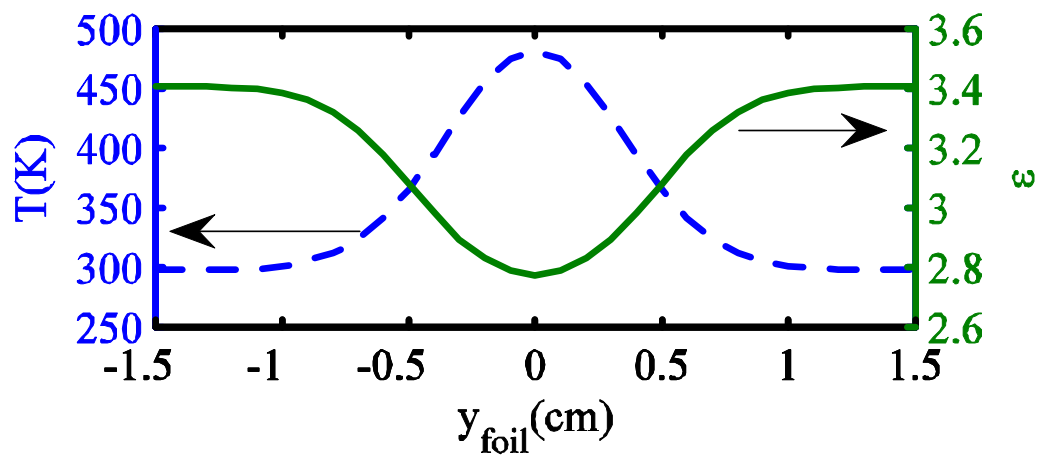

Figure 4.4: Mid-shot Kapton foil temperature and sample permittivity profiles along a center vertical strip for a Gaussian current profile with an $r_{r m s}$ of $0.5 \mathrm{~cm}$.

With the temperature profile determined, the required permittivity can be estimated. For Kapton, the room temperature permittivity is $\sim 3.4$ in the relevant photon energies [4]. The moderate imaginary component is ignored for simplicity. A simple Debye model [5] is used for the temperature dependence due to a lack of experimental data and results in the permittivity profile shown in Figure 4.4. The Debye model is strictly not applicable at the optical frequencies of concern, but useful nevertheless for illustrative purposes here. More accurate permittivity models or data at optical frequencies as a function of temperature are needed and are part of ongoing work. The Debye model gives:

$$
\frac{\varepsilon-1}{\varepsilon+2}=\beta\left(\alpha(\omega)+\frac{\mu^{2}}{3 k_{b} T}\right)
$$

Where $\beta$ is $8.4 \times 10^{37} \mathrm{~J} / \mathrm{C}^{2} \mathrm{~m}^{2}, \alpha(\omega)$ is $\sim 3 \times 10^{-39} \mathrm{C}^{2} \mathrm{~m}^{2} / \mathrm{J}$ for the photon energies of concern, and $\mu \sim 5.3 \times 10^{-30} \mathrm{Cm}$ for Kapton. These values were determined from the limited amount of experimental data available $[4,6]$.

If required, the detailed temperature profile in Figure 4.4 using the realistic current profile could be added to the flat top shot-to-shot temperature model from Section II to get an approximate temperature profile which accounts for shot history. 


\section{Detector Response}

Taking the results above and simulating the viewing cone of the diagnostic gives the detector response along the center vertical strip for each incident electron, again assuming no beam divergence and temperature. Figure 4.6 illustrates this response curve for different f-numbers. For each point along the vertical strip, the solid angle to the instrument is determined. The OTR emission from that point is then approximated by a trapezoidal integration with limits determined by the solved solid angle. The electrons near the center emit less light due to both the anisotropic nature of OTR and the lowered permittivity from the Debye model. These response curves are then multiplied by the same input current profiles used to determine the temperature of the foil in order to produce the complete synthetic diagnostic solution.

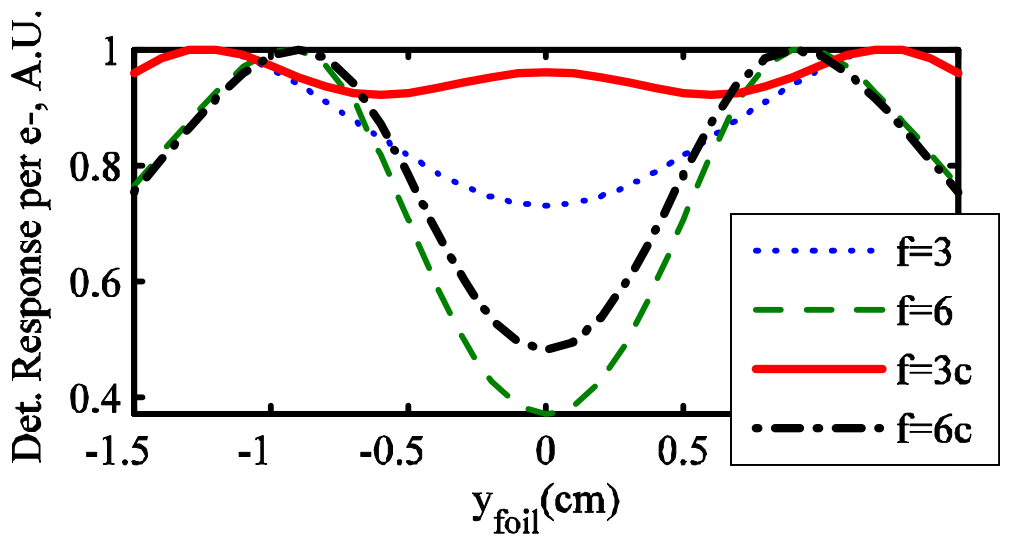

Figure 4.6: Detector response per incident beam electron along a Kapton center vertical strip using different f-number settings. The first two curves use the $\varepsilon$ profile in Figure 4 , while the last two denoted by the additional ' $c$ ' in the legend assumes a constant $\varepsilon=3.4$. The responses have been normalized for comparison.

Section IVb: Results

Multiplying the detector responses with the Gaussian beam current profile results in the sample synthetic spot-size profiles shown in Figure 4.7. This simulated profile is plainly wider than the input beam current profile. As shown, this additional width is caused by both the anisotropic nature of the OTR emission and decreased emission due to the foil temperature profile. For the higher f-number cases, hollow spot-size profiles with twin peaks are predicted. The finite divergence and temperature of a real beam would however eliminate some or all of the hollowness. The divergence and temperature of the 
beam will become important when they dominate or significantly change the beam-foil impact angles (and hence the effective OTR observation angle for the diagnostic for each electron) discussed above and illustrated in Figure 4.1.

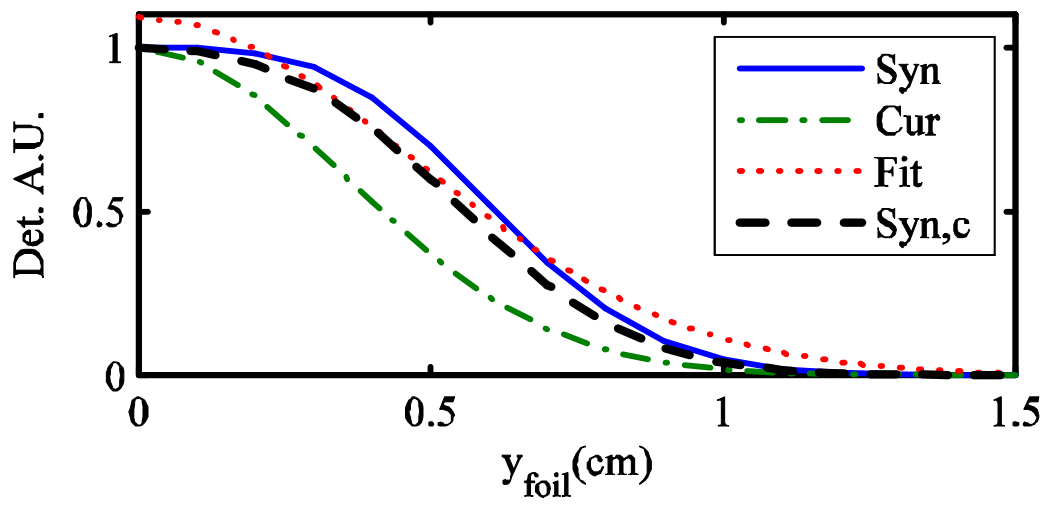

Figure 4.7: $\mathrm{f}=6$ Synthetic diagnostic profiles for a Gaussian beam current profile using the response curves in Figure 6. A Gaussian fit of the first diagnostic profile gives an $r_{r m s} \sim 0.66 \mathrm{~cm}$, indicating an observed $r_{r m s}$ that is $\sim 30 \%$ greater than the real beam $r_{r m s}$. The last curve shows the synthetic profile using a constant $\varepsilon=3.4$. It has a fitted $r_{\mathrm{rms}} \sim 0.62$ $\mathrm{cm}, 24 \%$ greater than the real beam $r_{\text {rms. }}$.

In general, the simulations predict that the observed spot-size will be larger than the real beam spot-size, especially at high f-numbers. Figure 4.8 plots the simulated spot FWHM on Kapton versus the input beam current profile FWHM. The FWHM is used here since Gaussian fits do not work well with hollow or flat profiles, and is equal to $2.35 \mathrm{r}_{\text {rms }} / 2^{0.5}$ for a Gaussian. It should be noted that this $\mathrm{r}_{\mathrm{rms}}-\mathrm{FWHM}$ relation is not necessarily correct for the simulated image profiles since the profiles are not typically real Gaussians, especially the ones that are hollow. Anyhow, using these types of curves prepared with some estimate or knowledge of the real beam divergence and accurate permittivity data, the real current radius can be calculated quickly based on observed spot size data. The data for the $\mathrm{f}=3$ curves lie very close to the ideal reference line. Temperature effects for these Kapton cases are small but noticeable compared with the geometric factors in terms of FWHM; however, they are significant in terms of profile shape. Figure 4.9 plots the simulated spot size FWHM on metallic foils versus the input beam current profile FWHM; these foils are immune to temperature based effects but are still affected by geometric factors. Again, for the higher f-number cases, the observed FWHM is higher than the beam FWHM. The $\mathrm{f}=3$ spot sizes for low to moderately size ( $\sim 2 \mathrm{~cm}$ FWHM) 
match the beam FWHM relatively well, but do not match the reference line as well as the $\mathrm{f}=3$ Kapton case. However, from an operations point of view, the $\mathrm{Al}$ is still preferred since it produces significantly more signal, can be quite robust compared with the Kapton when coated onto quartz, and can be simulated accurately since roughness and Cerenkov issues are eliminated. Because the Al coated foils in the experiments in Section III were smooth, the simulations results in Figure 4.9 are quantitatively applicable to experiments with OTR data taken at the waist of a beam with small emittance.

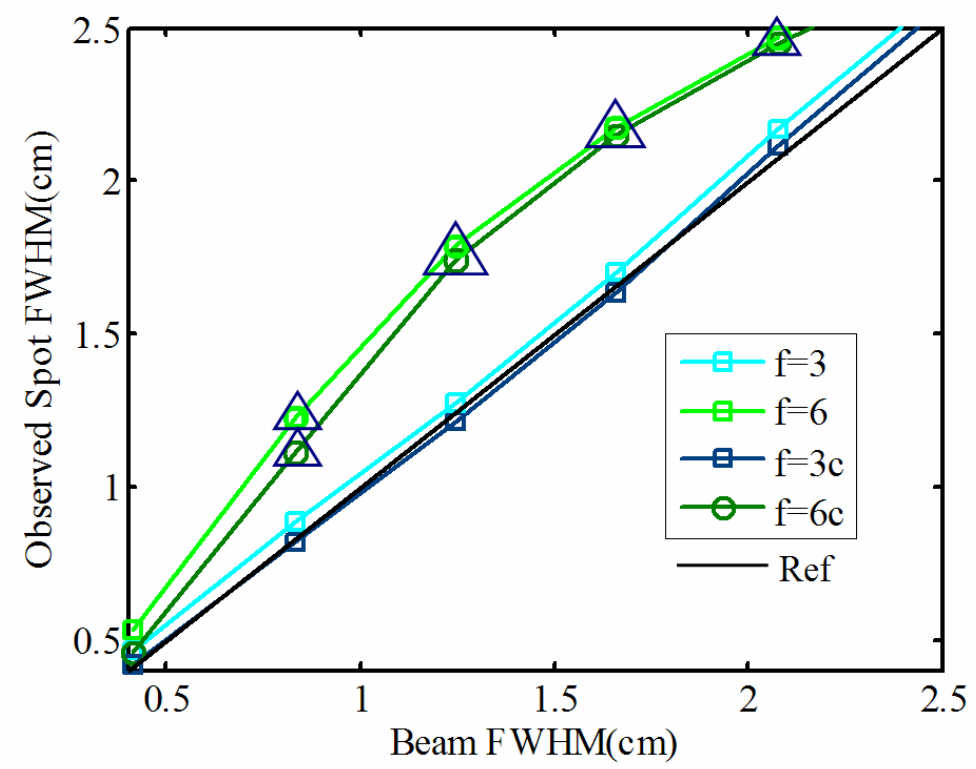

Figure 4.8: Observed FWHM versus Beam FWHM for Gaussian FXR current profiles at different f-numbers for Kapton foils with and without modeled temperature effects. The first two curves (without the 'c' indicator) on the legend include temperature effects. The FWHM plotted here corresponds to an RMS radius of 0.25 to $1.5 \mathrm{~cm}$. The low f-number setting gives the best FWHM match. A perfect match would result in the black "Ref" line. Points inside the blue triangles indicate simulated emission profiles that are hollow. 
LLNL-TR-402621

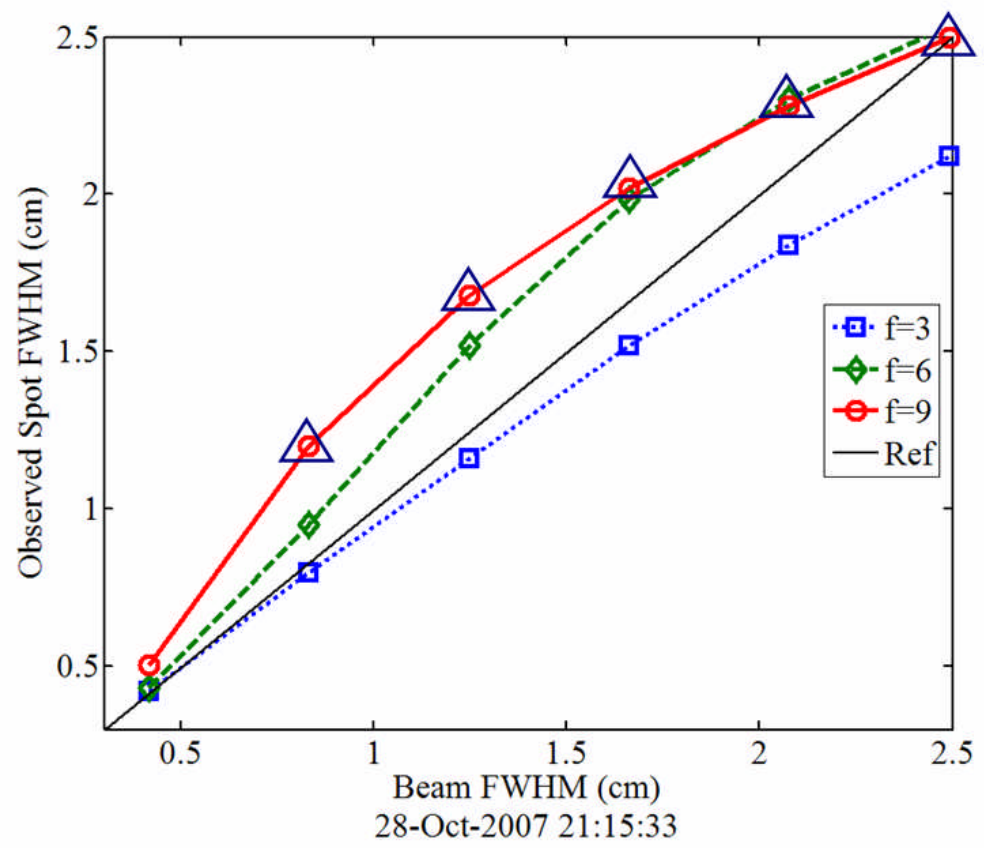

Figure 4.9: Observed FWHM versus Beam FWHM for Gaussian FXR current profiles at different f-numbers for metallic foils. The FWHM plotted here corresponds to RMS radius of 0.25 to $1.5 \mathrm{~cm}$. The low f-number setting gives the best FWHM match. A perfect match would result in the black 'Ref' line. Points inside the blue triangles indicate simulated emission profiles that are hollow.

Lastly, a preliminary simulation of the total counts versus spot RMS radius was made for Kapton foils and compared with experiment. Figure 4.10 gives the prediction and compares them with previous data taken by Houck. The simulation and experimental data shows a reasonable match, as long as temperature based effects were included with the geometric effects. Interestingly the total counts at first increased as a function of increasing radius but later decreases as the beam becomes larger. The rising part of the curve is primarily due to temperature effects. On the decreasing side, the total counts decreases as the beam gets too big since the outer part of the foil, where most of the beam now impacts, has a sightline to the camera that is now tilted large enough to move significant away from the peak OTR emission at $\sim 1.7$ degrees. The data in Figures 1.3 and 1.5 also shows similar trends. Some key issues with this comparison are that the experimental data contain many shots that did not have their centroid in the center of the foil as assumed by the simulation, and again the assumption in the simulation of a smooth foil. Nevertheless, the match shows that at least qualitatively results from the synthetic diagnostic assuming smooth foils are relevant. 


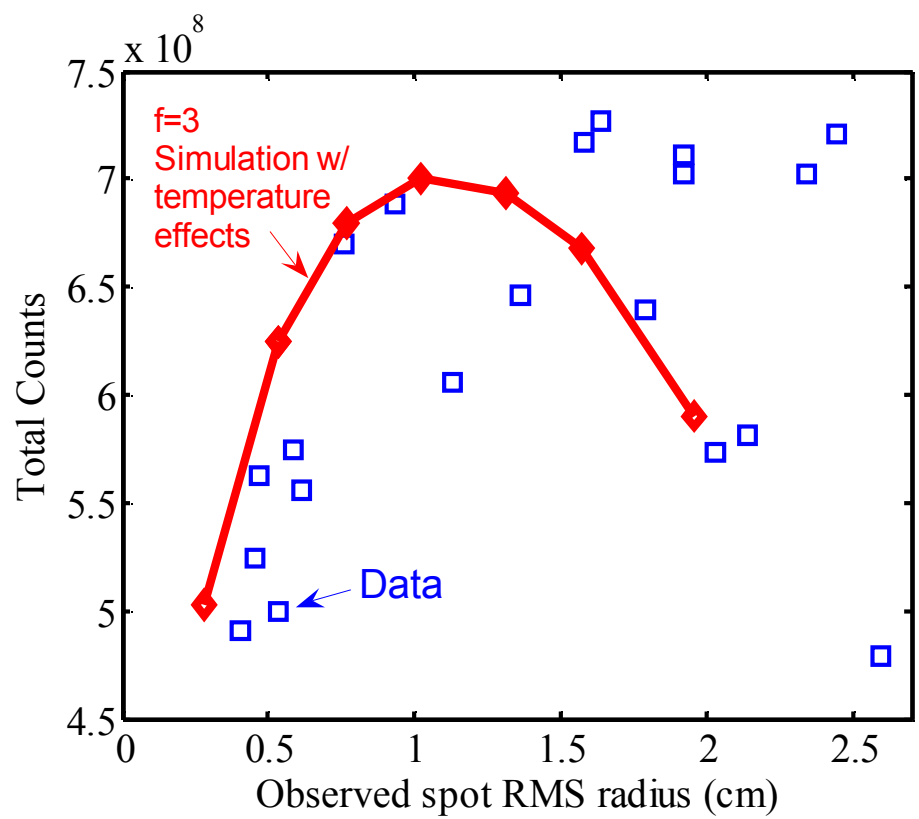

Figure 4.10: Comparison of Kapton experimental counts trends versus simulation. The simulated curve was scaled for the comparison.

\section{Section IV References}

[1] V. Tang et al., "Characterization of a high current induction accelerator electron beam via optical transition radiation from dielectric foils", Proc. 22nd Part. Acc. Conf., 2007. [2]M. L. Ter-Mikaelian, High Energy Electromagnetic Processes in Condensed Media, Wiley-Interscience, New York, 1972, Equations 25.16 and 25.18.

[3]B. Gitter, “Optical Transition Radiation”, CAA-TECH-NOTE-internal-\#24, UCLA, 1992.

[4]E. T. Arakawa et al., J. Appl. Phys., 52, 1981.

[5]P. Atkins, Physical Chemistry, 6th ed., W.H. Freeman and Co., New York, 1998, pg. 656.

[6]DuPont Company, "Summary of Properties for Kapton Polyimide Films", www.dupont.com 
LLNL-TR-402621

\section{Section V: Conclusions, suggested future work and operating procedures}

During FY07, new analysis, simulation, and experimental approaches were utilized to interpret OTR spot data from both dielectric foils such as Kapton and metal coated foils. Significant new understanding involving the diagnostic and FXR operational issues was achieved. Here, a conclusion and summary are given along with suggested future work and operating procedures for the FXR imaging diagnostics.

An overview of the FXR imaging diagnostics used for both imaging the beam current density profile and the beam divergence was first given along with problems associated with interpreting the diagnostic data. Cerenkov and OTR emission were examined in order to identify the types of radiation involved for the two FXR imaging diagnostic operating with dielectric foils. For the Wiggle Probe, Cerenkov radiation was estimated to be the primary source of detected emission. Based on some ray-tracing analysis, in comparison the Diagnostic Cross imaging system was estimated to see mostly OTR, even for the slightly roughened or frosted foils employed. The analysis showed that theoretically Cerenkov radiation should not be detected by the camera for a smooth foil. Previously uncovered interpretation problems associated with the image spot size at the Diagnostic Cross were specifically reviewed (similar observations were made at the Wiggle Probe); it was theorized that the spot profile on the foil did not correlate directly with the beam current density profile and that this was primarily due to foil heating that changed the permittivity of the foil non-uniformly during and after a shot. This would change the OTR emission profile since it is dependent on the permittivity. Hence the spot RMS radius would not be exactly the beam RMS radius and the total detected counts would not be conserved. Calculations confirmed that for a randomized OTR source, the total counts as a function of beam RMS radius would increase for a permittivity that decreased with temperature; the trend is independent of the exact shape of the current density profile. If randomized Cerenkov radiation was dominant instead, the total counts as a function of beam RMS radius would instead decrease. Previous data analysis for Kapton foils by Houck gave the OTR trend assuming a decreasing permittivity with 


\section{LLNL-TR-402621}

temperature for optical frequencies; permittivity data at lower frequencies for Kapton showed a decreasing permittivity with temperature.

A new post-shot Matlab toolset was written to facilitate spot image analysis and used on the old data sets; the results showed increasing total counts as a function of spot RMS radius for Kapton and also now for frosted quartz. An additional effect thought to affect the total counts and radius interpretation was the anisotropy of the OTR emission since the roughened or frosted dielectric foils appear only to isotropize the emission moderately. Lastly, beam perturbation from the foils is also possible in some cases. These analyses and theories prompted several related efforts to better understand the FXR imaging diagnostics.

The first involved attempts to understand the temperature behavior of the foils during and in-between shots. Detailed models including conduction and thermal radiation showed that radiation was the key heat loss mechanism and that for repeat shots, a steady-state temperature was reached with relatively few shots. The thermal radiation loss mechanism explained the survivability of the OTR foils. For thin foils, like the Kapton used, shot-to-shot temperature build-up was estimated to be small since in a lot of cases most of the heat can be radiated away within a minute or so. This new temperature model can be used to prevent damage to the foils and for emission simulations. Additionally some helium furnace tests were perform in order to observe visual changes in Kapton foils as a function of temperature.

The second involved several series of repeat shots on FXR using roughened Kapton, frosted quartz, and Al coated quartz foils. These experiments were designed to confirm temperature based effects on the OTR emission from dielectric foils and the results were to be used for benchmarking simulations of the diagnostic. A methodology was devised to isolate the shot-to-shot based temperature effects from the ones caused by instantaneous heating, and to determine if shots repeated properly. The experimental results are still under analysis but there is evidence for some temperature based effects. The data shows that there were notable beam reproducibility issues on FXR when warm- 


\section{LLNL-TR-402621}

up shots were not used. This is supported by the frosted quartz and Al coated quartz data sets. The number of warm-up shots needed is estimated to be minimally $\sim 15$ shots. Hence overall the experiments were not optimum but the identification of possible repeatability issues is an important conclusion. In general the Al coated quartz performed the best in terms of signal level, while the Kapton performed the worst. Lastly, some experiments with the Al coated quartz were performed at the Wiggle Probe angle ( $\sim 75$ degrees), but the signal levels were low.

The third effort concerned a full blown synthetic diagnostic or simulation of the diagnostic detector response for a set of shot parameters. The ultimate goal of this diagnostic was to provide corrections between the spot RMS radius and the real beam RMS radius, and to identify and quantify the different effects that are involved. The simulations so far involved ideal non-divergent beams hitting smooth Kapton foils and metal or metal coated foils at 45 degrees, and showed that a significant effect in the correction between spot FWHM and beam current FWHM was the anisotropic nature of the OTR emission, especially for high f-number optical setup where the acceptance angle of the viewing cone is small. The temperature effects were also included using a simple Debye model, and were shown to have a smaller effect on the radius interpretation compared with the geometric or anisotropic effect. However, a simulation of the total counts versus spot RMS radius for previous Kapton data gave a reasonable match and showed that temperature based effects are important in the prediction of count trends. Spot FWHM as a function of beam current FWHM plots were made for both Kapton and metallic foils and could be used for data interpretation. In general, for low f-numbers (i.e. $\sim 3$ ), the correction between the spot radius and real beam radius was small, generally less than $15 \%$. The incorporation of finite emittance and scattering for rough surfaces in the simulation, assuming the emission is still OTR, would further reduce that difference since they tend to reduce the effective anisotropy.

The work done up to this point suggests that the discrepancies seen between the beam transport simulations and the previous experimental data, assuming the simulations are accurate, might be due to both shot reproducibility issues on FXR and the slight to 
moderate differences between the spot radius and real beam radius, especially if the OTR diagnostic was operated at high f-numbers at the Diagnostic Cross. The Wiggle Probe data are still left to be examined in detail but raise some questions; if the primary radiation detected was randomized Cerenkov and since the permittivity of Kapton decreases with temperature, the total counts as a function of beam radius should decrease, not increase as Houck found previously. One possibility would be to do the diagnostic simulation for Cerenkov radiation as was done for OTR to see how significant geometric effects are and if they dominate over temperature based effects. The current Wiggle Probe might be more sensitive to geometric or anisotropic type effects compared with operations at the Diagnostic Cross. The problem with the simulation approach, as noted, is that an accurate model for surface roughness and scattering would be needed for good quantitative predictions since the foil is neither completely smooth or completely rough (i.e. giving perfect random scatter). If possible, a modified Wiggle Probe operating with an Al coated Kapton foil at 45 degrees should be considered. The machine could be protected from the metal coating if an acceptable transparent dielectric material with a permittivity close to one is available to additionally coat or cover the metal. Another possibility is to examine phosphor material sandwiched between thin Kapton sheets, assuming the phosphor emits isotropically. A directly different approach would be to incorporate some sort of material that can randomize the Cerenkov radiation fully; this would of course add some OTR to the detected emission.

Concerning modifications to operating procedures based on the research so far, it is suggested that only metallic or metallically coated foils be used in future experiments at the Diagnostic Cross. The optical system should be operated with as low an f-number as possible (i.e. 2.8). This essentially eliminates interpretation issues for the diagnostic since there are no temperature dependent effects and the emission is only OTR. The diagnostic response can also be rigorously simulated as shown and hence accurate beam profiles could be easily extracted. The temperature model in Section II with radiation can be used to ensure an adequate shot interval to prevent damage to the coating and foil. 
Experiments should be performed with the Al coated foil setup to study the beam reproducibility issues. Additionally, a radius scan for this foil should be done which would allow a comparison of the total counts versus radius curve with simulations. Dielectric foils could be further studied after these tasks. Specifically, it would be interesting to redo the repeat scans using smooth Kapton and quartz after the repeatability issues are ameliorated. Signal levels remain a concern however.

On the simulations and analysis side, additional future work includes simulating the OTR diagnostic at 75 degrees, incorporating finite emittance and divergence of the beam, calculating a total counts curve for metallic foils, and reexamining the FWHM curves in Figures 4.8-4.9 using a proper RMS average for the higher f-number cases so that old data taken at those f-numbers can be further studied. Lastly, the literature search for permittivity data as a function of temperature at optical frequencies for the dielectric foils of interest should continue.

\section{Acknowledgments}

The authors thank Jan Zenthler, Jim Dunlap, Blake Kreitzer, Sean Watson and the entire FXR staff for diligent operation of the beam for the experiments in this report, and for useful discussions. Fruitful discussions concerning scattering from rough foil surfaces were conducted with Gary Guethlein and Roger Richardson. The lead author thanks Tim Houck and Mike Ong for their continued support and encouragement. 